\title{
HALO STREAMS IN THE SEVENTH SLOAN DIGITAL SKY SURVEY DATA RELEASE
}

\author{
R. Klement ${ }^{1}$, H.-W. Rix ${ }^{1}$, C. Flynn ${ }^{2}$, B. Fuchs ${ }^{3}$, T. C. Beers ${ }^{4}$, C. Allende Prieto 5,6 , D. Bizyaev ${ }^{7}$, H. Brewington $^{7}$, \\ Y. S. LeE $^{4}$, E. Malanushenko ${ }^{7}$, V. Malanushenko ${ }^{7}$, D. Oravetz ${ }^{7}$, K. Pan ${ }^{7}$, P. Re Fiorentin ${ }^{1,8}$, A. Simmons $^{7}$, \\ AND S. SNEDDEN ${ }^{7}$ \\ ${ }^{1}$ Max-Planck-Institut für Astronomie, Königstuhl 17, D-69117 Heidelberg, Germany; klement@mpia.de, rix@mpia.de \\ ${ }^{2}$ Tuorla Observatory, V ais al antie 20, FI-21500 PIIKKI O, Finland; cflynn@ utu.fi \\ ${ }^{3}$ Astronomisches Rechen-Institut am Zentrum für Astronomie Heidelberg, Mönchhofstraße 12-14, D-69120 Heidelberg, Germany; fuchs@ari.uni-heidelberg.de \\ ${ }^{4}$ Department of Physics and Astronomy, CSCE: Center for the Study of Cosmic Evolution, and JINA: Joint Institute for Nuclear Astrophysics, Michigan State \\ University, E. Lansing, MI 48824, USA; beers@pa.msu.edu, lee@pa.msu.edu \\ ${ }^{5}$ McDonald Observatory and Department of Astronomy, The University of Texas, 1 University Station, C1400, Austin, TX 78712-0259, USA; \\ callende@astro.as.utexas.edu \\ ${ }^{6}$ Mullard Space Science Laboratory, University College London, Holmbury St. Mary, Surrey RH5 6NT, UK \\ ${ }^{7}$ Apache Point Observatory, Sunspot, NM 88349, USA; snedden@apo.nmsu.edu, dmbiz@ apo.nmsu.edu, hjbrew@apo.nmsu.edu, viktorm@apo.nmsu.edu, \\ elenam@apo.nmsu.edu, doravetz@apo.nmsu.edu,kpan@apo.nmsu.edu, asimmons@apo.nmsu.edu \\ ${ }^{8}$ Department of Physics, University of Ljubljana, Jadranska 19, 1000 Ljubljana, Slovenia; paola.refiorentin@ fmf.uni-1j.si \\ Received 2008 December 20; accepted 2009 April 1; published 2009 May 26
}

\begin{abstract}
We have detected stellar halo streams in the solar neighborhood using data from the seventh public data release of the Sloan Digital Sky Survey (SDSS), which includes the directed stellar program Sloan Extension For Galactic Understanding and Exploration (SEGUE). In order to derive distances to each star, we used the metallicity-dependent photometric parallax relation from Ivezić et al. We examine and quantify the accuracy of this relation by applying it to a set of globular and open clusters observed by the SDSS/SEGUE and comparing the resulting sequence to the fiducial cluster sequences obtained by An et al. Our final sample consists of 22,321 nearby $(d \leqslant 2 \mathrm{kpc})$, metal-poor $([\mathrm{Fe} / \mathrm{H}] \leqslant-0.5)$ main-sequence stars with six-dimensional estimates of position and space velocity $(\vec{r}, \vec{v})$. We characterize the orbits of these stars through suitable kinematic proxies for their "effective" integrals of motion, angular momentum, eccentricity, and orbital polar angle and compare the observed distribution to expectations from a smooth distribution in four $[\mathrm{Fe} / \mathrm{H}]$ bins. The metallicities provide an additional dimension in parameter space that is well suited to distinguish tidal streams from those of dynamical origin. On this basis, we identify at least five significant "phase-space overdensities" of stars on very similar orbits in the solar neighborhood to which we can assign unambiguously peaked $[\mathrm{Fe} / \mathrm{H}]$ distributions. Three of them have been identified previously, including the halo stream discovered by Helmi et al. at a significance level of $\sigma=12.0$. In addition, we find at least two new genuine halo streams, judged by their kinematics and $[\mathrm{Fe} / \mathrm{H}]$, at $\sigma=2.9$ and 4.8 , respectively. For one stream the stars even show coherence in the configuration space, matching a spatial overdensity of stars found by Juric et al. at $(R, z) \approx(9.5,0.8) \mathrm{kpc}$. Our results demonstrate the practical power of our search method to detect substructure in the phase-space distribution of nearby stars without making a priori assumptions about the detailed form of the gravitational potential.
\end{abstract}

Key words: Galaxy: kinematics and dynamics - solar neighborhood

Online-only material: color figures

\section{INTRODUCTION}

The phase-space distribution of stars in the solar neighborhood encodes enormous amounts of information on the present dynamical state and the formation history of the Milky Way. A key role in this context is played by the existence of substructure in the phase-space distribution of stars, caused by stellar streams or moving groups, that is, groups of stars moving on similar orbits in the Milky Way's gravitational potential. Such moving groups have been known to exist in the velocity distribution of nearby stars for some time (Proctor 1869; Lindblad 1925; Eggen 1996, and references therein). Stellar streams, together with their chemical and dynamical properties, can be used to constrain various scenarios of the hierarchical buildup of the Milky Way (e.g., Helmi et al. 1999) as well as its gravitational potential (e.g., Antoja et al. 2008).

Moving groups in the solar neighborhood emerge for several reasons. The simplest case is an agglomeration of stars that were born in the same molecular cloud and only recently dissolved. In this case, the stars keep on moving in the direction of the once-bound cluster, until phase-mixing washes out their common orbital signature. While this scenario seems to be valid for some stellar streams (e.g., HR1614; De Silva 2007), the chemical and chronological properties of the largest moving groups are incompatible with it. For example, Chereul \& Grenon (2001) reported an age range of $0.5 \mathrm{Gyr}$ to more than 2$3 \mathrm{Gyr}$ for Hyades supercluster, along with a rather large velocity dispersion, which they identified with the presence of several subgroups. Such subgroups have also been found by Dehnen (1998), who used subsamples of young and old stars based on their spectral types. He discovered an asymmetric drift relation for the moving groups, in the sense that those only present in the red subsamples (old stars) have larger radial velocity components, and lag with respect to the local standard of rest (LSR), than those also containing blue (younger) stars. In other words, old moving groups move on more eccentric orbits. To explain this observation, Dehnen (1998) proposed that these streams consist of stars that have been trapped onto nearly resonant orbits that oscillate about their parent resonant orbits, while the latter slowly change their eccentricity along with 
the nonaxisymmetric potential. This interpretation is based on suggestions already made by Mayor (1972) and Kalnajs (1991). The latter tried to explain the bimodal velocity distribution of the Sirius (moving radially inward) and Hyades (moving radially outward) streams by putting the Sun at the position of the outer Lindblad resonance (OLR) of the Galactic bar. However, Famaey et al. (2004) later pointed out that these streams are better explained as stars on horseshoe orbits that cross-corotate in the rest frame of spiral density waves (for more details, see Sellwood \& Binney 2002). They further argued that the clusters of coeval stars that have traditionally been connected to these streams would have been picked up by the spiral waves along with field stars of different ages, and therefore are just moving in these kinematic groups by chance. De Simone et al. (2004) also found that spiral waves can produce kinematic structures similar to those observed in the solar neighborhood, although they attributed this more to disk heating rather than radial migration.

Dehnen (2000) and Fux (2001) later used the position of the Hercules stream, which lags the LSR by $\sim 50 \mathrm{~km} \mathrm{~s}^{-1}$, to constrain the inclination angle and position of the OLR of the Galactic bar. Similarly, Quillen \& Minchev (2005) found that placing the Sun near the 4:1 inner Lindblad resonance with a two-armed spiral density wave could account for the velocityspace positions of the Hyades and Coma Berenices moving groups. Very recently, simulations of the birth and evolution of disk stars in a Milky Way potential including axisymmetric components for the disk, the bulge and halo, spiral arms, and a bar, were able to reproduce the shape of the Hercules, Coma Berenices, Hyades and Sirius moving groups in velocity space (Antoja et al. 2008). These examples show that the velocity distribution, as well as the age and chemical composition of dynamical streams in the solar neighborhood, can be used as tracers of the Galactic potential.

A third scenario for the formation of a stellar stream is a tidally disrupting cluster or satellite galaxy that deposits its debris on similar orbits (i.e., a "tidal" or "halo stream"). Helmi \& White (1999) performed simulations of disrupting satellites crossing the solar neighborhood and showed that the debris loses its spatial coherence completely over a Hubble time. In contrast, the stream stars clump together in velocity space, resembling classical moving groups. The reason is that stars in a single stream obey the collisionless Boltzmann equation, a special case of Liouville's theorem, which states that the phase-space density of a stellar subpopulation is conserved at any given phase-space point. Initially, the stream stars are located in a small phasespace volume. However, as the spatial components of the stars disperse with time, they become more focused in their velocity components. Although in practice the velocity dispersion of a tidal stream tends to increase with time, due to the effect of phase mixing (Helmi \& White 1999), such tidal streams will still form coherent features in velocity space, because to be in the solar neighborhood at the same time the azimuthal velocities of the stars must be similar. This has been confirmed with a number of different data sets, and stellar halo streams have been identified in the kinematic distribution of solar neighborhood stars (Helmi et al. 1999; Chiba \& Beers 2000; Navarro et al. 2004; Arifyanto \& Fuchs 2006; Helmi et al. 2006; Dettbarn et al. 2007; Klement et al. 2008, hereafter Paper I). Tidal streams also conserve the so-called integrals of motion of their progenitor, energy and angular momentum, allowing their recovery even if the halo has undergone complete mixing (Helmi \& de Zeeuw 2000). Trying to confine Milky Way streams into a small range of energies could yield a best fit to the gravitational potential.
The primary goal of this paper is to search for substructure in the solar neighborhood that can be attributed to tidal halo streams. Helmi et al. (1999) predicted that $~ 500$ kinematically cold streams might exist in the solar neighborhood, yet only a few have been detected so far. Currently, however, the Sloan Digital Sky Survey (SDSS; York et al. 2000) and its extensions, which have thus far obtained spectra for over 400,000 stars, represent the most extensive database collected to date to increase the number of detected halo streams. As has been shown by simulations from various authors (e.g., Helmi \& de Zeeuw 2000; Peñarrubia et al. 2006; Choi et al. 2007), a straightforward approach to finding stellar streams would be in the space of the integrals of motion $\left(E, L, L_{z}\right)$, which are immune to phase mixing. In practice, however, the integrals of motion are not uniquely defined, because the potential is not exactly known. Furthermore, the error bars on six-dimensional measurements are drastically "anisotropic" across the different components. We therefore want to explore search strategies in a modified integrals-of-motion space that match the data and error bars.

This paper is organized as follows. Section 2 describes our data set, our methods for deriving distances, comparisons of our adopted photometric parallax relation with cluster fiducial sequences, and estimates of space velocities for the stars. Section 3 describes our strategy for searching for streams in the solar neighborhood. We apply our methods in Section 4, pointing out where several of our streams overlap with previously detected examples. We consider the potential effects of systematic distance errors in Section 5. Section 6 discusses our methods for the determination of the statistical significance associated with stream detection. In Section 7, we present four new likely streams and confirm the detection of three others identified in previous work (adding additional members to these structures). Our conclusions and a brief discussion are presented in Section 8.

\section{THE DATA}

SDSS-I was an imaging and spectroscopic survey that began routine operations in 2000 April and continued through 2005 June. The SDSS, and its extensions, uses a dedicated $2.5 \mathrm{~m}$ telescope (Gunn et al. 2006) located at the Apache Point Observatory in New Mexico. The telescope is equipped with an imaging camera and a pair of spectrographs, each of which is capable of simultaneously collecting 320 medium-resolution $(R=2000)$ spectra over the $7 \mathrm{deg}^{2}$ field of view (FOV), so that on the order of 600 individual target spectra and roughly 40 calibration-star and sky spectra are obtained on a given spectroscopic "plug-plate" (York et al. 2000). The imaging camera (Gunn et al. 1998) contains an imaging array of 304 megapixel CCDs and astrometric arrays that measure fluxes for calibration with standard astrometric catalog stars. The flux densities of objects observed are measured almost simultaneously in five bands $[u, g, r, i, z]$, with effective wavelengths of [3540 , $4760 \AA, 6280 \AA, 7690 \AA, 9250 \AA$ ] , respectively (Fukugita et al. 1996; Gunn et al. 1998; Hogg et al. 2001). The camera sweeps the sky in great circles (in a drift scan mode) and a point on the sky passes the filters in the order of $r, i, u, z, g$. The brightness limit where the imaging camera saturates is at $g \sim 14 \mathrm{mag}$. The completeness at this magnitude is $\sim 99.3 \%$ for point sources (Ivezić et al. 2001); it drops to 95\% at magnitudes of [22.1, $22.4,22.1,21.2,20.3] .{ }^{9}$ The SDSS photometry is accurate to

\footnotetext{
9 These values have been derived by comparing multiple scans of the same area obtained during the commissioning year with a typical seeing of $1^{\prime \prime} .5 \pm 0.1$
} 
0.02 mag rms at the bright end, ${ }^{10}$ with well controlled tails of the error distribution (Ivezić et al. 2003). Astrometric positions are accurate to about $0^{\prime \prime} .1$ per coordinate for sources brighter than $r \sim 20.5$ mag (Pier et al. 2003). Morphological information from the images allows point source-galaxy separation to $r \sim 21.5$ mag (Lupton et al. 2002).

One of three subsurveys carried out during the first extension of the SDSS, known as SDSS-II, the Sloan Extension for Galactic Understanding and Exploration (SEGUE), ran from 2005 July to 2008 June. SEGUE obtained some 250,000 medium-resolution spectra of stars in the Galaxy, selected in order to explore the nature of stellar populations from $0.5 \mathrm{kpc}$ to $100 \mathrm{kpc}$ (Yanny et al. 2009). These data, along with the substantial numbers of suitable stars observed during the course of SDSS-I, allow the derivation of the full six-dimensional phase-space distribution of the various components of the Milky Way. Stellar physical parameters $\left(T_{\text {eff }}, \log g,[\mathrm{Fe} / \mathrm{H}]\right)$, based on SDSS photometry and spectroscopy, are derived by the application of the SEGUE Stellar Parameter Pipeline (SSPP) described by Lee et al. (2008a, 2008b) and Allende Prieto et al. (2008).

We start the sample selection for the present study from all stars targeted for spectroscopy by SDSS/SEGUE with a signalto-noise ratio $(\mathrm{S} / \mathrm{N})$ greater than 10 , accepted photometry in all five bands, and estimates for the radial velocity, $[\mathrm{Fe} / \mathrm{H}]$, and proper motions. Note that the requirement for determinations of $[\mathrm{Fe} / \mathrm{H}]$ is essentially one on effective temperature (or color), as the present SSPP provides confident estimates of metallicity over the range $4500 \mathrm{~K} \leqslant T_{\text {eff }} \leqslant 7500 \mathrm{~K}$. These stars have been taken from the seventh data release (Abazajian et al. 2009); their proper motions have been corrected for a systematic error that occurred in the data reduction procedure (Munn 2008). There are a number of repeated observations, either for quality assurance or from reuse of photometric calibration stars by several plugplates. These repeats are independent observations and are listed separately in the SDSS Catalog Archive Server, with different identification numbers. We only keep one object per position on the sky, to which we assign a radial velocity and stellar parameters averaged over all repeats. Figure 1 shows the sky coverage of the resultant sample of 154,888 stars. These data cover a large, almost contiguous area in the Northern Galactic Cap plus three stripes in the South Galactic Cap.

\subsection{Distance Estimates}

The majority of stars spectroscopically targeted by SDSS-I are main-sequence stars (or metal-poor main-sequence turnoff stars used as calibration objects; 99\%; Finlator et al. 2000). Although the targets spectroscopically selected by SEGUE explicitly include giants, their fraction (based on spectroscopic surface gravity estimates) remains low. Only $8.8 \%$ of these stars have $\log g<3.5$, the surface gravity where we divide between dwarfs and giants. This is a bit more stringent than the separation made, for example, by Ivezić et al. (2008) at $\log g=3$, but we wish to ensure that only late-type dwarfs and subdwarfs are selected. ${ }^{11}$ Presuming a sample dominated by main-sequence stars, we can apply a photometric parallax relation to derive distances. Because we want to concentrate on a wide range of metal-poor stars, the effect of metallicity

\footnotetext{
10 This value is determined using repeated observations of 3,000,000 point sources over time spans ranging from $3 \mathrm{hr}$ to three years.

11 A subdwarf is defined as a star with luminosity 1.5-2 mag lower than that of a solar-metallicity main-sequence star of the same spectral type.
}

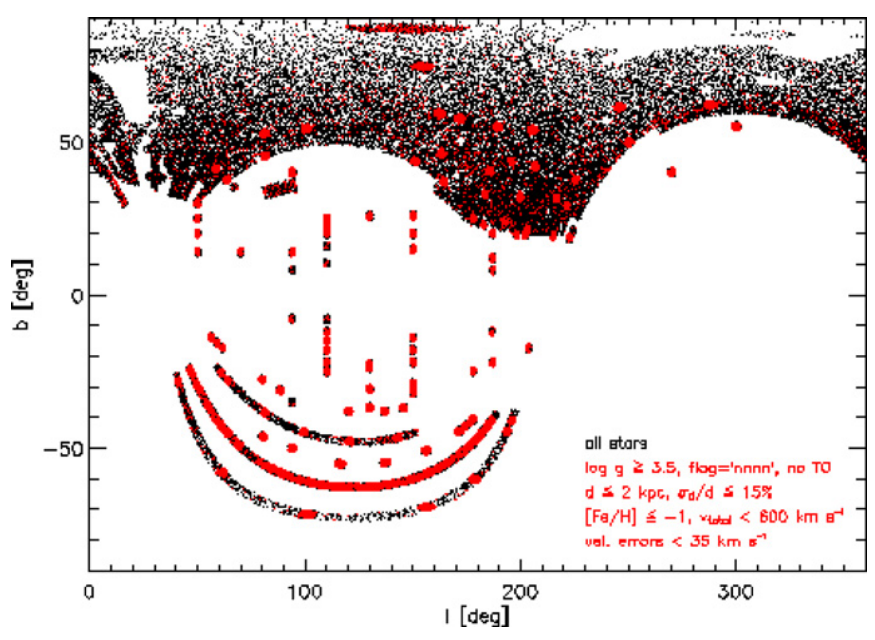

Figure 1. Sky coverage of our sample of SDSS/SEGUE stars. Each star is plotted individually. The red data points represent the positions of metal-poor stars that meet the selection criteria of our final sample (see Section 2.2). Note the sparse sampling obtained during SDSS-I vs. the focused sampling of the SDSS-II/SEGUE pointings.

on the absolute magnitude at a given color becomes important. We have spectroscopic metallicities available for each star, so we are motivated to adopt a photometric parallax relation that explicitly accounts for metallicity over a wide range of colors.

Such a relation has been derived by Ivezić et al. (2008). The shape of their color-magnitude relation, $M_{r}^{0}(g-i)$, is constrained by simultaneously fitting SDSS photometry data for five globular clusters, normalized to the same arbitrary magnitude scale by requiring the same median magnitude $(r=0)$ for stars in the color range $0.5<g-i<0.7$. By assuming that this shape depends only on color, and not metallicity, and its normalization depends only on metallicity, and not color, the absolute magnitude offset of each cluster from the mean relation can be expressed as a function of metallicity. The absolute magnitude of a star is then calculated as

$$
M_{r}(g-i,[\mathrm{Fe} / \mathrm{H}])=M_{r}^{0}(g-i)+\Delta M_{r}([\mathrm{Fe} / \mathrm{H}]) .
$$

With distances adopted from Harris (1996) and six additional open and globular cluster data from Vandenberg \& Clem (2003), they derive the following absolute magnitude correction:

$$
\Delta M_{r}([\mathrm{Fe} / \mathrm{H}])=4.50-1.11[\mathrm{Fe} / \mathrm{H}]-0.18[\mathrm{Fe} / \mathrm{H}]^{2} .
$$

The correction (Equation (2)) suggests an offset from the mean relation of 4.5 for solar metallicity stars, due to the scaling to $r=0$ described above. Ivezić et al. (2008) further expanded the mean photometric parallax relation to the color range $0.2<g-i<4$.0 by using constraints from trigonometric parallaxes given in Bochanski et al. (2008), additional cluster data observed in the SDSS from Clem et al. (2008), and an age correction for turnoff stars. The result is a fifth-order polynomial:

$$
\begin{aligned}
M_{r}^{0}(g-i)= & -5.06+14.32(g-i)-12.97(g-i)^{2} \\
& +6.127(g-i)^{3}-1.267(g-i)^{4}+0.0967(g-i)^{5},
\end{aligned}
$$

which, together with Equations (1) and (2), is our adopted photometric parallax relation.

We test the validity of this relation for our sample using different approaches, as described below. 


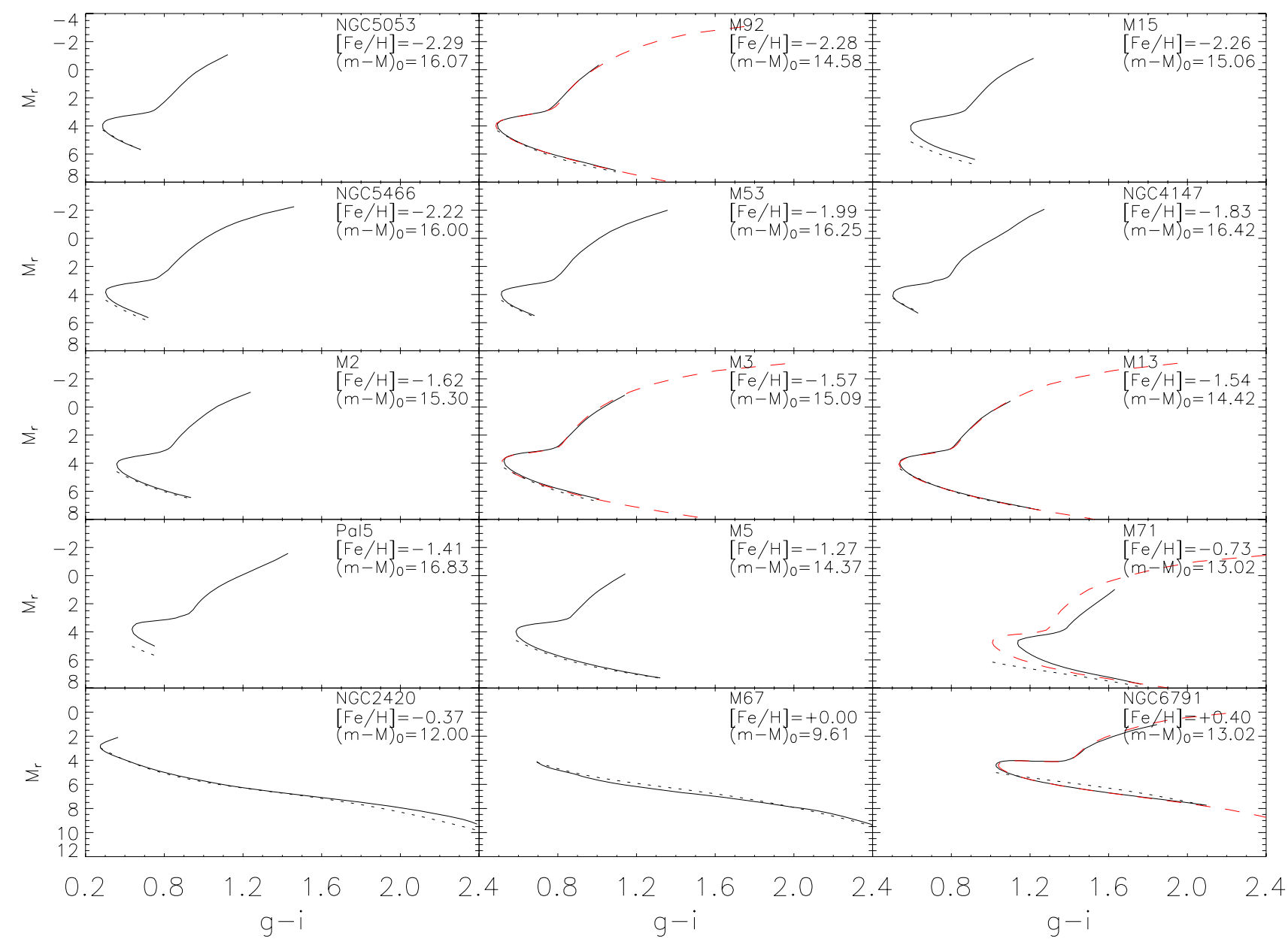

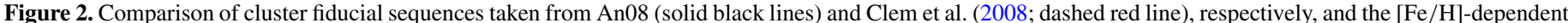

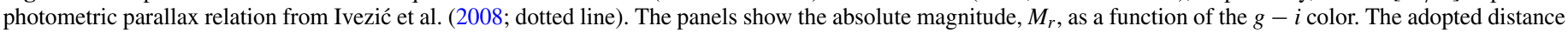
moduli and metallicities are taken from various sources in the literature (see An08, Section 2, for all references).

(A color version of this figure is available in the online journal.)

\subsubsection{Comparison of the Photometric Parallax Relation with Cluster Fiducial Sequences}

An et al. (2008, hereafter An08) have used crowded-field photometry techniques to analyze SDSS/SEGUE imaging data for 17 globular and three open clusters, and determined fiducial sequences from their color-magnitude diagrams (CMDs). These sequences give the $r$-band magnitude as a function of either $u-g, g-r, g-i$, or $g-z$ color. This is the first time that cluster fiducial sequences have been evaluated in the native SDSS ugriz system, allowing for tests of the photometric parallax relation from Ivezić et al. (2008) without the need to rely on color transformations from other systems.

We use 15 of the cluster fiducials and compare them to the sequences derived from Equations (1)-(3). In addition, we consider the fiducial sequences from Clem et al. (2008) for five clusters, after transforming them onto the ugriz system using the transformations given by Tucker et al. (2006). These sequences have been shown to match the An08 fiducials within the errors of the photometric zero points. Because they were obtained from observations with various integration times, the Clem et al. sequences extended over a broader magnitude range than the An08 sequences. We calculated the absolute magnitude, $M_{r}$, for each cluster by adopting the distance moduli and metallicities as given in An08. The results are shown in Figure 2, sorted by decreasing cluster metallicity.
For most of the clusters, the photometric parallax relation of Ivezić et al. (2008) fits the fiducial main sequences remarkably well. In the case of nearly all the metal-poor clusters with $[\mathrm{Fe} / \mathrm{H}]<-1.0$, the difference between the absolute magnitude predicted by the photometric parallax relation, $M_{r \text {,phot }}$, and the absolute magnitude given by the cluster fiducial sequences, $M_{r \text {, cluster }}$, stays below $\sim 0.2 \mathrm{mag}$ for $g-i \gtrsim 0.4$. There are three exceptions to this trend. One is M15, where the turnoff is slightly redward of $g-i=0.4$ and the absolute magnitude offset drops roughly from $M_{r, \text { phot }}-M_{r \text {,cluster }}=0.48$ mag at $g-i=0.51$ to $0.36 \mathrm{mag}$ at $g-i=0.77$. For Palomar 5 , the discrepancy is more than 0.7 mag across the main sequence. However, this cluster is known to be in the process of tidal disruption (Odenkirchen et al. 2001; Grillmair \& Dionatos 2006) and is sparsely populated in the observations; contamination by foreground and background stars is possible. Also, the color range spanned by the main sequence is very small compared with the giant branch, and it may not extend far enough from the turnoff for the photometric parallax relation to be valid. The An08 fiducial sequence for M71 has to be taken with caution, because according to these authors the zero points for the M71 photometry were very uncertain, and there was a strong contamination by likely background stars. The uncertain fiducial sequence could thus account for the offsets.

We find it more descriptive to express the systematic differences between the photometric parallax relation and the cluster 


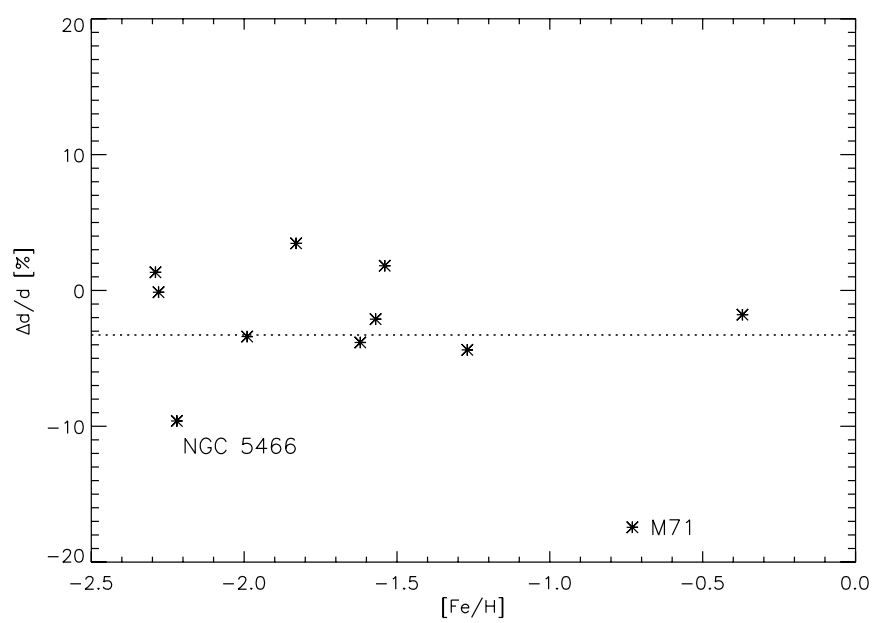

Figure 3. Relative distance errors, $\left.\Delta d / d=\left(d_{\text {phot }}-d_{\text {cluster }}\right) / d_{\text {cluster }}\right)$, vs. metallicity, $[\mathrm{Fe} / \mathrm{H}]$, derived from 11 of the 13 clusters that lie in the metallicity range $-2.0<[\mathrm{Fe} / \mathrm{H}]<-0.3$. The errors of each cluster have been averaged over the color range $(g-i)_{\text {TO }}+0.05<g-i<1.5$. The two clusters M15 and Pal5 have not been considered, while M71 has been kept, because it has a sequence measured by Clem et al. (2008) with deep photometry. The dotted line indicates the distance offset averaged over all 11 clusters (including M71), which we adopt as the systematic distance error for the Ivezić et al. (2008) photometric parallax relation.

fiducial sequences through a distance offset rather than a magnitude offset. To derive an estimate of the systematic distance error of the photometric parallax relation, we average over the differences between the distance that is predicted by the relation, $d_{\text {phot }}$, and the distance given by the distance modulus of each cluster, $d_{\text {cluster }}$ To this end, we concentrate on the color range $(g-i)_{\text {TO }}+0.05<g-i<1.6$, where $(g-i)_{\text {Tо }}$ denotes the turnoff color, that is, the color at which a cluster's main sequence runs vertically. ${ }^{12}$ The color $g-i=1.6$ corresponds approximately to the reddest color in our sample. If available, we prefer the cluster sequences of Clem et al. (2008) for calculating $\Delta d / d$. After elimination of the two outliers mentioned above (M15 and Pal5), Figure 3 shows relative distance errors $\left.\Delta d / d=\left(d_{\text {phot }}-d_{\text {cluster }}\right) / d_{\text {cluster }}\right)$ over the range in metallicity $-2.0<[\mathrm{Fe} / \mathrm{H}]<-0.3$. Nine of the 11 cluster sequences considered suggest very small systematic errors, while the distances to NGC5466 and M71 are underestimated by $9.61 \%$ and $17.44 \%$, respectively. Averaging over all clusters, the systematic distance error is

$$
\left\langle\frac{\Delta d}{d}\right\rangle= \begin{cases}-3.28 \% \pm 1.78 \% & \text { including M71 } \\ -1.86 \% \pm 1.19 \% & \text { excluding M71 }\end{cases}
$$

We found no indication in Clem et al. (2008) that their sequence for M71 might be unreliable, so we will use the more conservative value of $-3.28 \%$ when considering the effects of velocity errors on our results. This value is indicated by the dotted line in Figure 3.

The photometric parallax relation of Ivezić et al. (2008) is constructed from stars that are redder than the main-sequence turnoff. Although the authors included a correction for age effects, and stated that their formula is valid over the color range $0.2<g-i<4.0$, we see from Figure 2 that the application of the relationship breaks down near the turnoff, the color of which depends on both metallicity and cluster age. Metal-poor F-type stars in the disk, for example, have a lifetime

$\overline{12}$ This is the bluest color for which a value of the fiducial sequence exists. considerably shorter than the age of the thin disk and are already in the turnoff phase. Having turnoff stars in our sample can result in additional systematic distance (and hence velocity) errors, which could lead to false stream detections. Theoretical isochrones could be used to determine the color at which stars of a given metallicity and age are in the turnoff phase. For example, the Girardi et at. (2004) ugriz isochrones predict a turnoff color of $g-i \approx 0.36(0.22)$ for a 13.5 Gyr old stellar population with $[\mathrm{Fe} / \mathrm{H}]=-1.0(-2.0)$. However, An08 have shown that the theoretical isochrones of Girardi et at. (2000) are not consistent with their fiducial sequences; the model colors for the main sequence are $2 \%-5 \%$ too blue. Therefore, we apply a color cut to our sample that is based on the location of the turnoff and the behavior of the distance errors in Figure 2 rather than on theoretical models.

We apply a stringent color cut in order to remove turnoff stars. We choose the color cuts depending on the metallicity of the stars as follows:

$$
g-i \geqslant\left\{\begin{array}{lll}
0.55 & \text { if } & {[\mathrm{Fe} / \mathrm{H}]>-1.0} \\
0.50 & \text { if } \quad-1.5<[\mathrm{Fe} / \mathrm{H}] \leqslant-1.0 \\
0.45 & \text { if }-2.0<[\mathrm{Fe} / \mathrm{H}] \leqslant-1.5 \\
0.40 & \text { if } \quad[\mathrm{Fe} / \mathrm{H}] \leqslant-2.0
\end{array}\right.
$$

These color cuts ensure that we select stars that are at least 0.05 mag redward of the cluster turnoffs in the corresponding metallicity bins. We do not use stars from the most metal-rich bin for our stream search, so we do not divide this bin further. The color cut, however, should be valid for stars up to solar metallicity. For reference, the Sun (a G2 star) has a $g-i$ color of $0.57 \pm 0.02$ (as measured from about 50 solar analogs; Holmberg et al. 2006).

To summarize, the photometric parallax relation from Ivezić et al. (2008) performs very well in fitting the main sequences for clusters with metallicities of $[\mathrm{Fe} / \mathrm{H}] \lesssim-0.3$. On average, it predicts distances that are incorrect by $-3.28 \% \pm 1.78 \%$. We thus adopt it to determine distances to all the dwarfs and subdwarfs $(\log g>3.5)$ in our sample. For more metalrich clusters $([\mathrm{Fe} / \mathrm{H}] \gtrsim-0.3)$ the uncertainties are generally higher, although they can vary along the main sequence. Further investigations with a larger number of clusters will be needed in order to better determine the accuracy of the relation for metal-rich stars.

\subsubsection{Three-Dimensional Velocities from $v_{\mathrm{rad}}, d_{\mathrm{phot}}$, and $\vec{\mu}$}

From the photometric parallax relation, Equations (1)-(3), and the dereddened $r$-band magnitudes, we calculate the distance to each star via

$$
d(\mathrm{kpc})=\frac{1}{100} \times 10^{0.2\left(r-M_{r}\right)} .
$$

We calculate the statistical distance error from Gaussian error propagation:

$$
\sigma_{d}=\frac{1}{5} d \ln 10 \sqrt{\left(\sigma_{r}\right)^{2}+\left(\sigma_{M_{r}}\right)^{2}}
$$

where $\sigma_{r}$ is given for each star in our sample. The estimated dispersion in the absolute magnitude, $\sigma_{M_{r}}$, follows from Equations (1)-(3), and the listed errors for $[\mathrm{Fe} / \mathrm{H}]$ and $g-i$. The mean statistical relative distance error of our sample is $7.58 \% \pm 0.01 \%$. An intrinsic (systematic) scatter of $3.28 \%$ (Equation (4)) is less than half of this value, and can be neglected relative to the statistical errors (see Section 5). Later, 


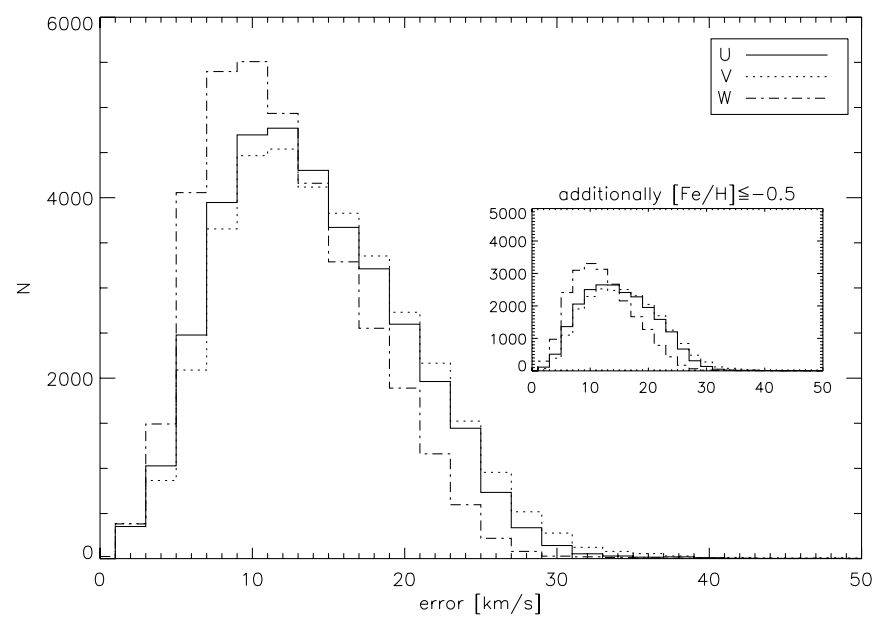

Figure 4. Distribution of errors for the velocity components $U$ (solid), $V$ (dotted), and $W$ (dash-dotted) for all stars with flag = "nnnn," $\log g \geqslant 3.5, d \leqslant 2 \mathrm{kpc}$, and $\frac{\sigma_{d}}{d} \leqslant 15 \%$. The small window shows the same distribution, exclusively for stars with the additional restriction $[\mathrm{Fe} / \mathrm{H}] \leqslant-0.5$.

we restrict our selection to stars with statistical distance errors $\frac{\sigma_{d}}{d} \leqslant 15 \%$ and distances $d \leqslant 2 \mathrm{kpc}$, so in the worst case, when we add statistical and systematic errors, the actual distance could be $2.37 \mathrm{kpc}$ instead of $2 \mathrm{kpc}$ (with a total distance error of $28.28 \%$ ).

Figure 4 shows the statistical velocity error distribution for 37,136 stars satisfying the SSPP flag = "nnnn" (indicating no peculiarities), $\log g \geqslant 3.5, d \leqslant 2 \mathrm{kpc}, \sigma_{d} / d \leqslant 15 \%$, and our color cuts (Equation (5)). Additionally, we show in the small window all 23,512 stars that also fulfill $[\mathrm{Fe} / \mathrm{H}] \leqslant-0.5$, because we cut at this metallicity for our final sample of metal-poor stars. The velocities and their errors have been calculated using the equations given in Paper I, Section 2.

The velocity error distribution rises to a peak around $10 \mathrm{~km}$ $\mathrm{s}^{-1}$ for $W$, and around $12-15 \mathrm{~km} \mathrm{~s}^{-1}$ for $U$ and $V$ and then falls off quickly. If we only select metal-poor stars from the above sample, the error distribution for $U$ and $V$ shifts to a peak around $13-15 \mathrm{~km} \mathrm{~s}^{-1}$. For $W$, the errors increase only slightly. This follows since the more metal-poor stars are on average farther away. We choose to accept errors up to $35 \mathrm{~km} \mathrm{~s}^{-1}$ for all three velocity components.

\subsection{A Sample of Metal-Poor Stars Within 2 kpc with Six-Dimensional Phase-Space Coordinates}

In order to obtain the best available sample of stars, regarding the accuracy of distances and suitability for our stellar stream search, we only keep stars satisfying the following criteria (the number in parentheses indicates the number of stars that are left after each step):

1. $\log g \geqslant 3.5$, in order to only select dwarfs and subdwarfs to which we can apply the photometric parallax relation (141,286);

2. SSPP flag = "nnnn," indicating that there are no suspected problems with derived atmospheric parameters $(118,584)$;

3 . distance $d \leqslant 2 \mathrm{kpc}$, because our search strategy requires nearby stars; also, the proper motions are more accurate for nearby stars $(44,484)$;

4. relative distance errors $\sigma_{d} / d \leqslant 0.15(44,087)$;

5 . total space velocity $v_{\text {total }}<600 \mathrm{~km} \mathrm{~s}^{-1}$, to exclude stars with apparently false proper motion measurements or distance estimates $(44,034)$;
6. velocity errors smaller than $35 \mathrm{~km} \mathrm{~s}^{-1}$ for $U, V$, and $W$ $(43,512)$;

7. $g-i \geqslant(g-i)_{\mathrm{TO}}$, where $(g-i)_{\mathrm{TO}}$ depends on the metallicity of a star according to Equation (5), to exclude turnoff stars $(35,864)$;

8. we restrict ourselves to $[\mathrm{Fe} / \mathrm{H}] \leqslant-0.5$, because we concentrate on thick-disk and halo substructure ${ }^{13}(22,321)$.

A distance cut of $2 \mathrm{kpc}$ is necessary, because our search strategy for streams assumes a constant rotation curve in the solar neighborhood, and that we can approximate the radial and rotational velocities by $U$ and $V$, respectively. In addition, we gain higher accuracy in the velocities, because the proper motions are more accurate for nearby stars. The spatial distribution of our sample is shown in Figure 1 as the red dots. As can be appreciated by inspection of this figure, our sample is distributed over the same region as the full DR-7 sample.

Although our final sample is only $14 \%$ of the original 154,888 stars, we have a sample of nearby metal-poor stars of both unprecedented quantity and quality (compare to, e.g., Helmi et al. 1999; Chiba \& Beers 2000; Arifyanto \& Fuchs 2006; Dettbarn et al. 2007). Figure 5 shows the color, distance, and metallicity distribution of our final sample. The distribution peaks at $g-i=0.7$ (the color of a G star) with a tail extending to $g-i \approx 1.3$ (K6-K7 stars). It is interesting that there remain some possible turnoff stars in the $g-r$ distribution at $g-r \approx 0.3$ for the most metal-rich bin $(-1.0<[\mathrm{Fe} / \mathrm{H}] \leqslant-0.5),{ }^{14}$ although this is not the case in the $g-i$ distribution. The $g-i$ color has (somewhat) better signal-to-noise properties than the $g-r$ color, except in the region of the main-sequence turnoff (Ivezić et al. 2008). In any case, the fraction of possible turnoff stars is so small that the influence of their systematically incorrect distances on our analysis can be neglected.

\section{SEARCH STRATEGY FOR STREAMS}

To search for stellar halo streams in our sample, we effectively look for overdensities in eccentricity-, orbital inclination-, and guiding center radius- (or, equivalently, angular momentum-) space. We adopt a method based on the Keplerian approximation of Dekker (1976) and outlined by Dettbarn et al. (2007). This method is a generalization of the formalism outlined in Paper I to identify stellar streams in RAVE data. There it was assumed that the azimuthal velocity of a star could be approximated by $V+V_{\mathrm{LSR}}$, thereby projecting the orbits into the meridional plane. Halo stars move on random, more eccentric orbits, and we can apply the same formalism if we group them together according to the inclination of their orbital planes.

We assume a spherical potential and neglect any asphericity of the dark-halo potential and flattening of the disk potential. This is justified by the work of Chiba \& Beers (2000), who showed that the distribution of halo stars in the space spanned by isolating integrals of motion in an aspherical Stäkel-type potential can be closely mapped into the integrals of motion space of a spherical potential. Also, even for stars that move in axisymmetric flattened potentials, $L_{\perp}$ is approximately conserved, and the orbits can be thought of as planar orbits precessing slowly around the $z$-axis (Binney \& Tremaine 1987). As an example we refer to Paper I, where it was shown that stellar thick- and

\footnotetext{
13 Also, higher metallicities are unreliable because of a calibration error that has only been fixed recently, and was not corrected in the Catalog Archive Server at the time we selected our stars (T. Beers 2008, private communication).

14 For the more metal-poor bins, the turnoff lies blueward of $g-r=0.3$.
} 


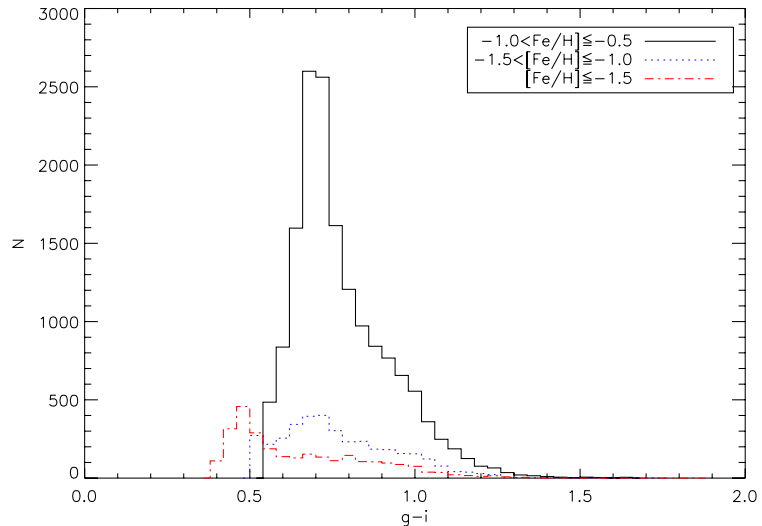

(a) $g-i$

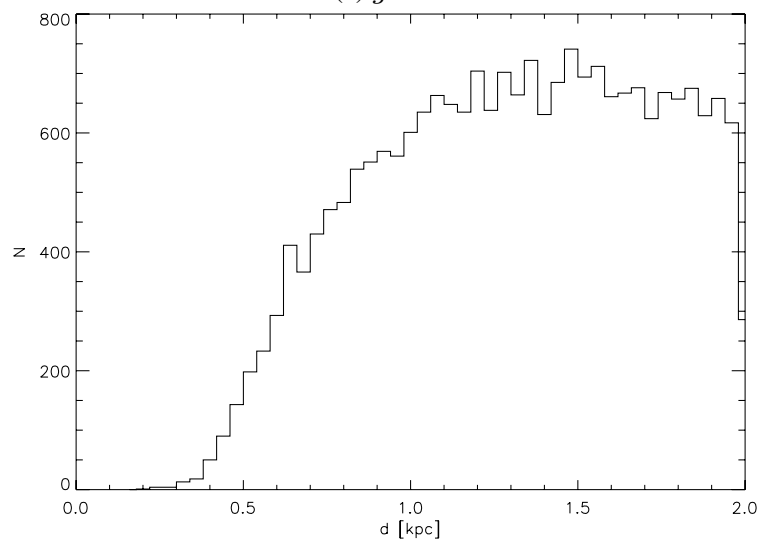

(c) $d$

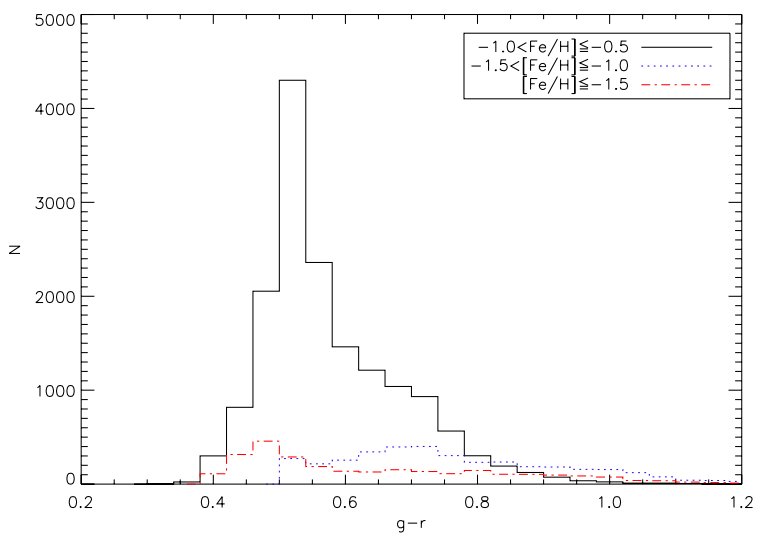

(b) $g-r$

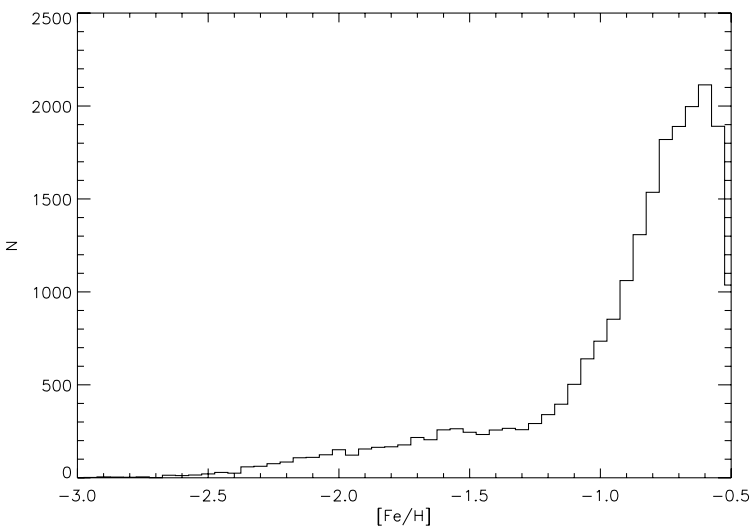

(d) $[\mathrm{Fe} / \mathrm{H}]$

Figure 5. Distribution of stars from our final sample in (a) colors $g-i$, (b) $g-r$, (c) distance $d$, and (d) metallicity [Fe/H]. Because of the applied color cuts, the sample consists of G- and K-type stars. Panels (a) and (b) display the color distribution for three bins in $[\mathrm{Fe} / \mathrm{H}]$. The different panels show that our sample is dominated by stars of the thick-disk population.

(A color version of this figure is available in the online journal.)

thin-disk streams detected in a projection of phase space are also clumped in $\left(L_{z}, L_{\perp}\right)$ space.

In a spherical potential, a star with Cartesian velocity components $(U, V, W)$ moves in a fixed orbital plane that is inclined by an angle $v$ relative to the direction toward the North Galactic Pole. The angle $v$ is given by

$$
v=\arctan \left(\frac{V+V_{\mathrm{LSR}}}{W}\right),
$$

and ranges from $0^{\circ}$ to $180^{\circ}$. Stars with polar angles $v>180^{\circ}$ are treated as moving on retrograde orbits in a plane with polar angle $v-180^{\circ}$. The azimuthal velocity of a star is

$$
V_{\mathrm{az}}=\sqrt{\left(V+V_{\mathrm{LSR}}\right)^{2}+W^{2}},
$$

so if the star is near the Sun we can approximate its total angular momentum by

$$
L=R_{\odot} \cdot V_{\mathrm{az}}=R_{0} \cdot V_{\mathrm{LSR}}
$$

For the last step, we have assumed a constant rotation curve. Finally, we express the eccentricity, $e$, of any stellar orbit as

$$
e=\frac{1}{\sqrt{2} V_{\mathrm{LSR}}} V_{\Delta E}
$$

where we have introduced the quantity

$$
V_{\Delta E}=\sqrt{U^{2}+2\left(V_{\mathrm{LSR}}-V_{\mathrm{az}}\right)^{2}} .
$$

The parameter $V_{\Delta E}$ parametrizes the difference between the energy of a star at the guiding center of its orbit and at the solar radius, and is a measure of its orbital eccentricity. Also, we have shown (B. Fuchs, unpublished) that $V_{\Delta E}$ is related to the radial action integral, and is robust against slow changes in the gravitational potential. ${ }^{15}$ Although the approximation 11 formally breaks down for highly eccentric orbits $(e>0.5$ Dekker 1976), stars on similar orbits will still be projected into the same region of phase space (Klement 2009). Looking for "overdensities" in $\left(V_{\mathrm{az}}, V_{\Delta E}, v\right)$ space is a practical way to find stellar streams, because we do not need to assume any expression for the gravitational potential and any prehistory of the stream. The first successful application of the generalized Keplerian approximation was by Dettbarn et al. (2007), who were able to rediscover the "H99" stream, originally found by Helmi et al. (1999) in the $\left(L_{z}, L_{\perp}\right)$ space.

We use metallicities $[\mathrm{Fe} / \mathrm{H}]$ to discriminate between stellar populations with different origins. We divide our sample into four subsamples (hereafter s1, s2, s3, s4) with decreasing metallicity:

1. s1: $-1.0<[\mathrm{Fe} / \mathrm{H}] \leqslant-0.5(15,856)$,

2. $\mathrm{s} 2:-1.5<[\mathrm{Fe} / \mathrm{H}] \leqslant-1.0(3676)$,

15 Using Dekker's (Dekker 1976) theory of Galactic orbits, the radial action integral can be expressed in the notation of Arifyanto \& Fuchs (2006) as

$$
J_{R}=-\sqrt{2} \pi R_{0}^{2} \kappa_{0}+\frac{\pi R_{0}^{3} \kappa_{0}^{2}}{\sqrt{E_{0}-E+\frac{1}{2} R_{0}^{2} \kappa_{0}^{2}}} \approx \frac{\pi R_{\odot}}{2 V_{\mathrm{LSR}}} V_{\Delta E}^{2} .
$$




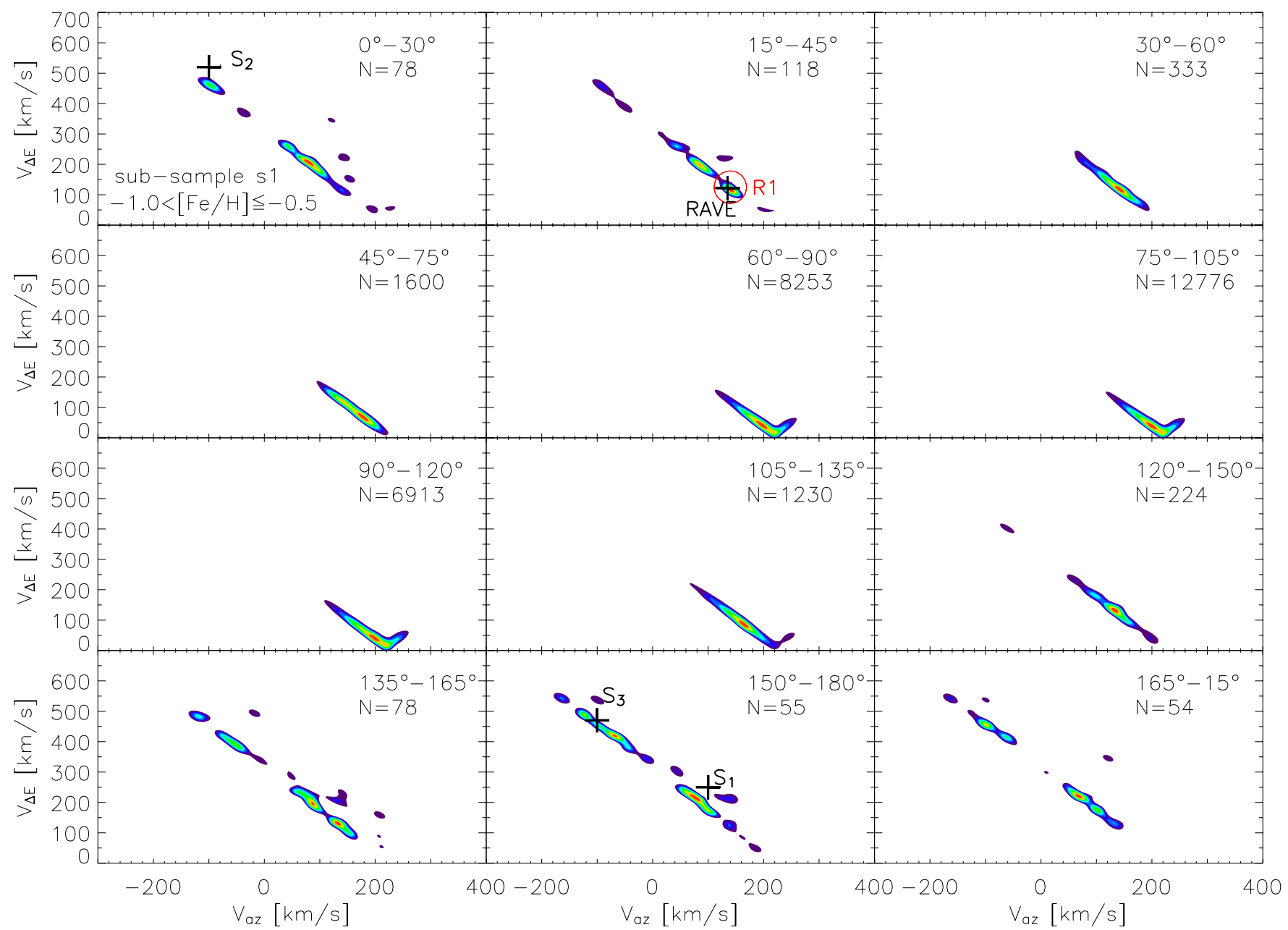

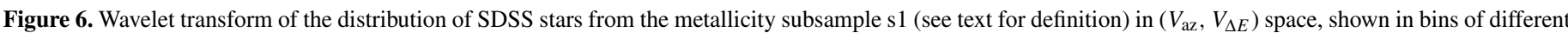

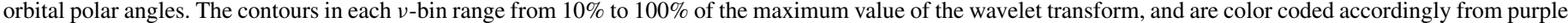

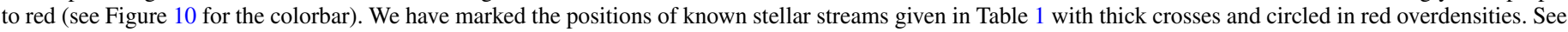
Section 7 for discussion.

3. $\mathrm{s} 3:-2.0<[\mathrm{Fe} / \mathrm{H}] \leqslant-1.5(1931)$,

4. s4: $[\mathrm{Fe} / \mathrm{H}] \leqslant-2.0(858)$.

The number in parenthesis is the number of stars in the corresponding subsample. We note that the typical metallicity estimates are uncertain to (at best) $\sim 0.25$ dex, making adjacent subsamples not entirely independent. For each $[\mathrm{Fe} / \mathrm{H}]$ bin, we collect the stars with similar orbital polar angles in small $v$-slices. We bin the polar angles into $30^{\circ}$ wide bins that overlap by $15^{\circ}$, thereby reducing bin-boundary effects on the results. We conduct the search for stellar streams within each $v$-slice in the space spanned by angular momentum and eccentricity or $\left(V_{\mathrm{az}}, V_{\Delta E}\right)$. To amplify the overdensities, we use a wavelet transform technique with a skewed Mexican-hat-shaped analyzing wavelet (see Paper I for more details). We set the scale parameter of the wavelet to $a=12 \mathrm{~km} \mathrm{~s}^{-1}$, comparable to the velocity errors, set the elongation parameter to $q=\sqrt{3}$, and employ cells in the $\left(V_{\mathrm{az}}, V_{\Delta E}\right)$ space of $3 \mathrm{~km} \mathrm{~s}^{-1}$ width on each side. The resulting contours of the wavelet transform are shown in Figures 6-9.

\section{PLACING SOLAR NEIGHBORHOOD STREAMS IN THE $\left(V_{\mathrm{az}}, V_{\Delta E}\right)$-PLANE}

As a first step in the analysis of the SDSS data, we explore whether we can find evidence in these data of the known streams believed to be of a tidal origin. We have taken the velocities of known halo streams from the literature and evaluated their velocities $V_{\mathrm{az}}, V_{\Delta E}$, and orbital inclinations, $v$. The results are given in Table 1 and marked with thick crosses in Figures 6-9. The feature marked ' $S_{2}$,' found by Dettbarn et al. (2007) as an overdensity of stars in the $\left(V_{\mathrm{az}}, V_{\Delta E}, v\right)$ space, has almost exactly the same properties as the " $\omega$ Cen"-stream; in fact, the signal of " $\mathrm{S}_{2}$ " in Figure 3 of Dettbarn et al. (2007) is extended across the $v$ range from 0 to $\sim 20^{\circ}$. Therefore, it is likely that " $\mathrm{S}_{2}$ " is in fact the " $\omega$ Cen"-stream, and we only label " $\omega$ Cen" in the plots.

We find strong evidence of the "H99," "S $\mathrm{S}_{3}$," and "RAVE" streams, which we discuss more in Section 7, but little or only minor hints for the existence of the other known streams.

\section{THE EFFECTS OF SYSTEMATIC DISTANCE ERRORS AND UNRESOLVED BINARIES}

To test for the possible effects of systematic distance errors on our results, we have added the $-3.28 \%$ systematic distance error that we found through comparison of the photometric parallax relation to cluster fiducial sequences in Section 2.1.1 to each star and then recalculated their velocities and the wavelet transform for a subset of stars. As expected, the effect of the additional $-3.28 \%$ errors is to slightly change the shape and 


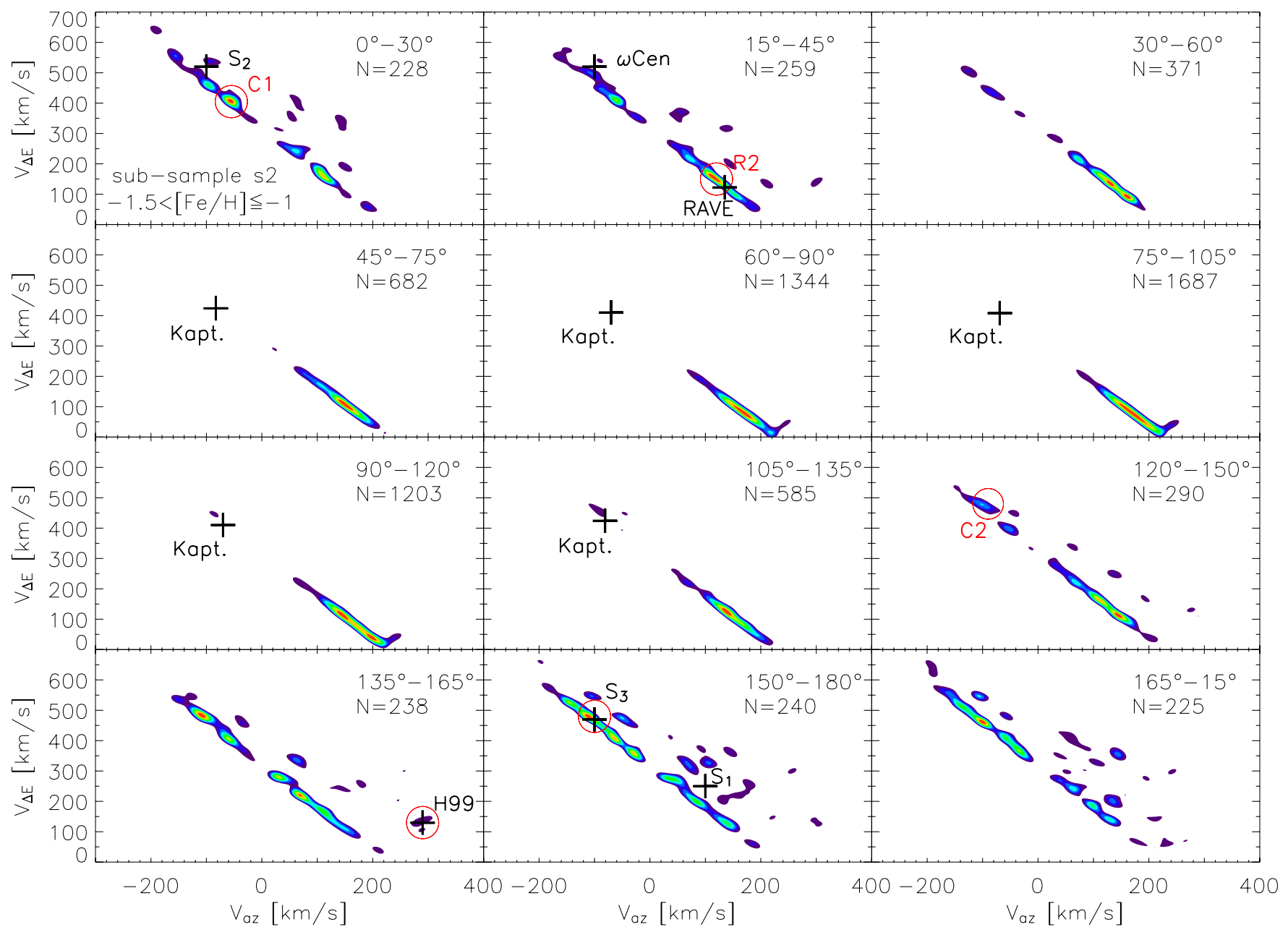

Figure 7. Same as Figure 6, but now for stars in the metallicity subsample s2.

relative "height" of some features, but the location of the overdensities does not change much. However, we also want to test if a much higher systematic error-say, $-10 \%$ - would effect the location of the overdensities. While we have shown in Section 2.1 .1 that a $-10 \%$ error is very unlikely at the lowmetallicty end of the photometric parallax relation, the error at the high-metallicity end $([\mathrm{Fe} / \mathrm{H}] \gtrsim-1.0)$ is much less constrained and a possibility for systematic errors on that level exists. This in turn may cause an overdensity that has a range of metallicities to be detected at varying positions in the $\left(V_{\mathrm{az}}\right.$, $V_{\Delta E}$ )-plane in different metallicity subsamples and be misinterpreted as multiple, adjacent, streams. As an example, Figure 10 shows contours of the wavelet transform for the distribution of stars from subsample s1 in the $v$-slice $15^{\circ}-45^{\circ}$ (a) without and (b) with additional $-10 \%$ distance errors. While the features remain close to their locations in $\left(V_{\mathrm{az}}, V_{\Delta E}\right)$, they change their shape and relative "height." We have investigated other subsamples and $v$-slices by adding similar distance errors (from $7 \%$ to $10 \%$ ) and found that the change in relative height of the overdensities is more prominent for stars on retrograde orbits. However, such stars are more represented in the lower metallicity bins, where we expect the systematic distance errors to be less severe. Given that the positions of the peaks roughly remain where they are in all examples we investigated, we can assume that the location of stellar streams in the $\left(V_{\mathrm{az}}, V_{\Delta E}\right)$-plane is not very sensitive to the expected systematic distance errors.
However, problems can arise when we want to distinguish adjacent streams that span more than one metallicity bin. Because the systematic errors can change with metallicity, the signal of a single stream can too and in this way produce apparently distinct, but adjacent, peaks.

Unresolved binary stars may affect our analysis through their effect on the photometric parallax relation and hence the distance determination. The number of multiple stellar systems expected in our sample of mainly $\mathrm{G}$ and $\mathrm{K}$ stars might be taken to be around $57 \%$ (probably lower) according to recent determinations (Lada 2006, and references therein). ${ }^{16}$ Unresolved multiple stars taken as single stars have too high observed luminosities and hence their distances are underestimated. Another effect arises, if the multiple stellar systems consists of stars of different masses, which leads to a shift in colors. To get a feeling of how the distances change when we mistake a binary system as a single star, we calculate by how much the apparent magnitude, $m$, changes. In the case of equal-mass binaries, the observed flux, $F$, would be only half as high for a single star.

$$
m=-2.5 \log \frac{F}{F_{0}}
$$

\footnotetext{
16 We note, however, that in our case of metal-poor stars the expected fraction of multiple stars drops further, because these stars tend to be older; hence, the systems had more time to disperse.
} 


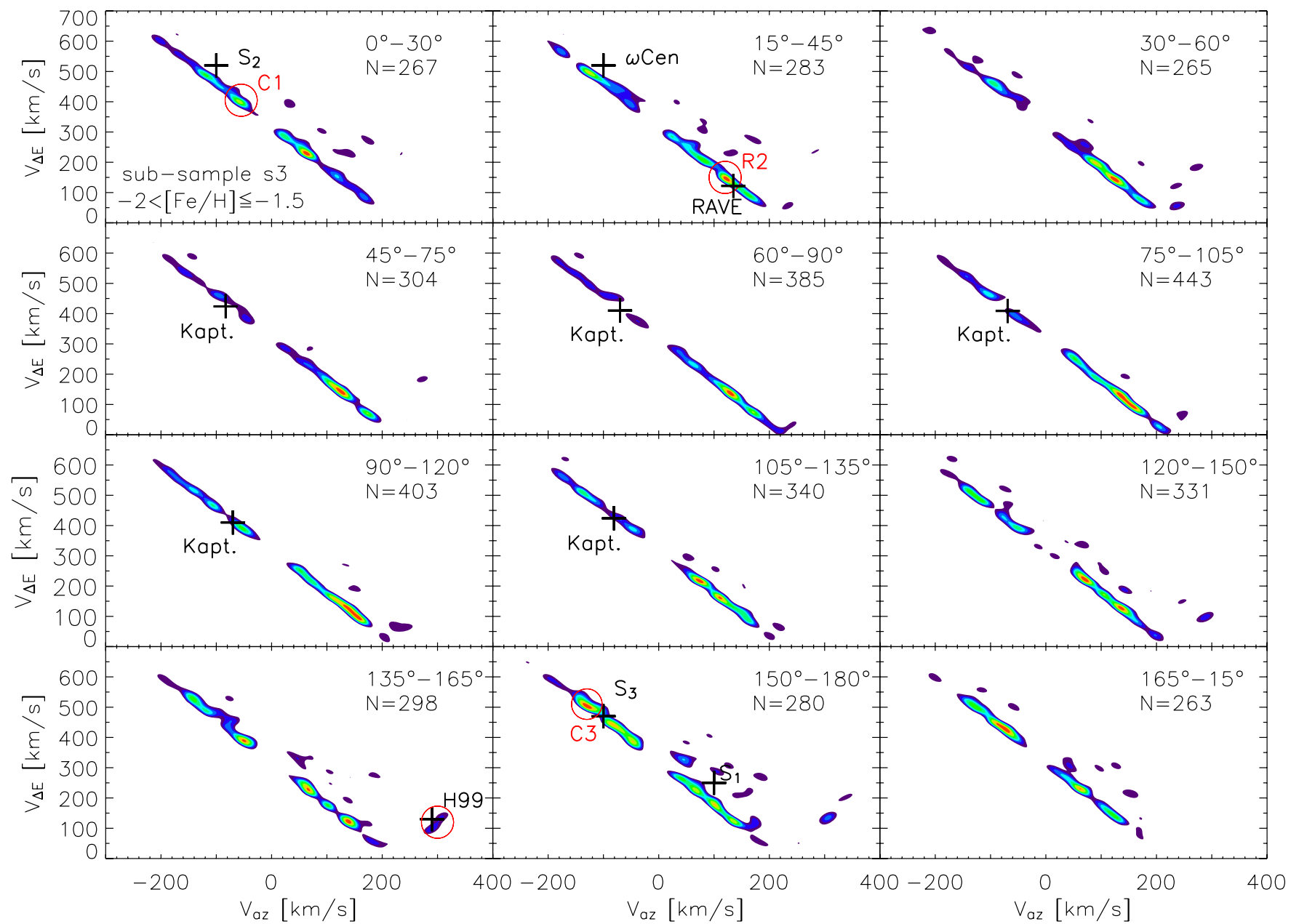

Figure 8. Same as Figure 6, but now for stars in the metallicity subsample s3.

is the definition of the apparent magnitude, where $F_{0}$ is some constant flux which gives $m=0$. It follows that the apparent magnitude that would be assigned to only the single star from the binary system, $m^{\prime}$, is given by

$$
m^{\prime}=-2.5 \log \frac{0.5 F}{F_{0}} \simeq m+0.75 \text {. }
$$

Together with Equation (6), it follows that in the worst case (equal-mass binaries) the distances for part of our sample are underestimated by $29 \%$. This is a systematic effect, and results in a change of the shape and relative "height" of the overdensities as discussed above. However, this effect might not be so severe, because (1) the change in distances has the opposite sign to the expected systematic error of the photometric parallax relation, (2) it affects approximately only half of our sample, and (3) most of nearby G-dwarf binary systems possess mass ratios that peak at much less than 1.0 (Duquennoy \& Mayor 1991). In addition to the systematic underestimation of distances, the radial velocities for multiple systems are scattered around the value of the dominant component in a statistical way due to the additional Doppler shift in the spectrum of the bound system. Taken together, these effects are hard to describe quantitatively, but they certainly only dilute the signatures of real streams. Hence, this works to make the numbers we identify as stellar streams a lower limit of what might exist.

\section{ESTIMATING THE SIGNIFICANCE OF OVERDENSITIES}

It is clear that overdensities in $\left(V_{\mathrm{az}}, V_{\Delta E}\right)$ can and will emerge through Poisson noise, especially in regions that are sparsely sampled by the data. As described in Paper I, Section 4.2, we can address this problem by performing Monte Carlo simulations with stars randomly drawn from a smooth distribution. From these we can build the residuals of the wavelet transforms of each individual simulation against the mean value for all wavelet transforms, which represents a smooth distribution over our search space. For each cell in $\left(V_{\mathrm{az}}, V_{\Delta E}\right)$, we can calculate the variance and use it to obtain a significance map of the overdensities. ${ }^{17}$

For the smooth halo and thick-disk components, we adopt the results of Chiba \& Beers (2000), who characterized the halo through a mean rotational velocity at $\langle\theta\rangle \approx 30 \mathrm{~km} \mathrm{~s}^{-1}$, with a radially elongated velocity ellipsoid $\left(\sigma_{U}, \sigma_{V}, \sigma_{W}\right)=$ $(141 \pm 11,106 \pm 9,94 \pm 8) \mathrm{km} \mathrm{s}^{-1}$. We further use $\langle\theta\rangle=$ $190 \mathrm{~km} \mathrm{~s}^{-1}$ and $\left(\sigma_{U}, \sigma_{V}, \sigma_{W}\right)=(46 \pm 4,50 \pm 4,35 \pm 3)$ for the thick disk, and adopt their estimated fraction of thick-disk stars in our subsample s1 as $80 \%$, in s2 as $30 \%$, and in s3 as $10 \%$. We are aware that the azimuthal drift for the thick-disk stars

\footnotetext{
17 Artificially high significance levels can emerge if we divide the residuals by a standard deviation which is less than 1 . For this reason, we set the variance to 1 in each cell where it is below this level.
} 


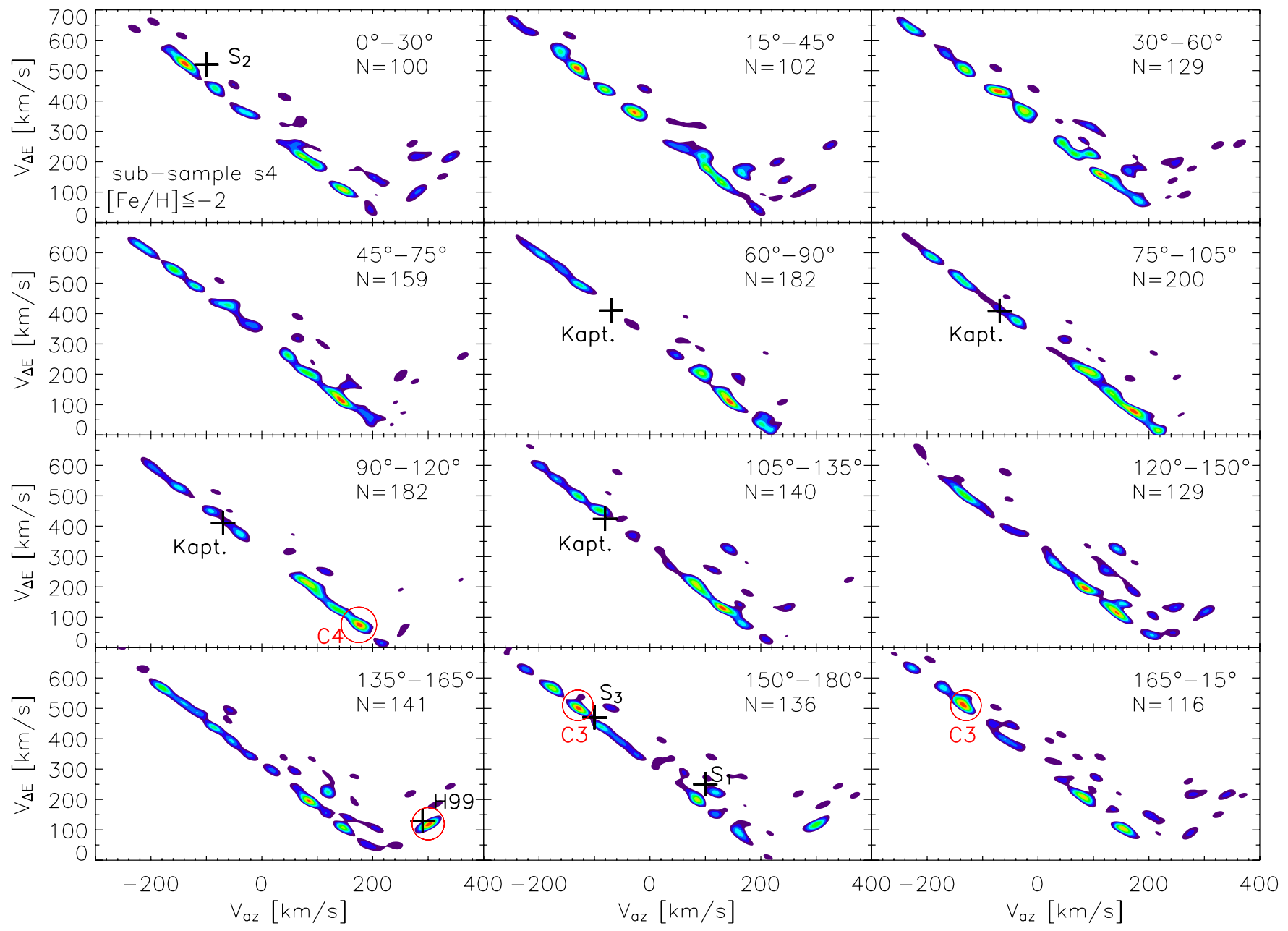

Figure 9. Same as Figure 6, but now for stars in the metallicity subsample s4.

Table 1

Velocities and Derived Effective Integrals of Motion for Known Solar Neighborhood Streams

\begin{tabular}{|c|c|c|c|c|c|c|c|c|}
\hline Parameter & Kapteyn $^{\mathrm{a}}$ & H99 ${ }^{b}$ & $\omega \mathrm{Cen}^{\mathrm{c}}$ & RHLS $^{\mathrm{d}}$ & $\mathrm{S}_{1}{ }^{\mathrm{e}}$ & $\mathrm{S}_{2}{ }^{\mathrm{e}}$ & $\mathrm{S}_{3}{ }^{\mathrm{e}}$ & RAVE $^{\mathrm{f}}$ \\
\hline$\langle|U|\rangle$ & 63 & 84 & $\ldots$ & 294 & $\ldots$ & $\ldots$ & $\ldots$ & 24 \\
\hline$\sigma_{|U|}$ & 54 & 65 & $\ldots$ & 6 & $\ldots$ & $\cdots$ & $\cdots$ & 15 \\
\hline$\langle V\rangle+V_{\mathrm{LSR}}$ & -69 & 130 & $\ldots$ & 99 & $\ldots$ & $\ldots$ & $\ldots$ & 61 \\
\hline$\sigma_{V}$ & 6 & 22 & $\ldots$ & 25 & $\ldots$ & $\ldots$ & $\ldots$ & 5 \\
\hline$\langle W\rangle$ & -16 & -240 & $\cdots$ & 239 & $\ldots$ & $\cdots$ & $\cdots$ & 121 \\
\hline$\sigma_{\mathrm{W}}$ & 67 & 24 & $\ldots$ & 24 & $\ldots$ & $\ldots$ & $\ldots$ & 30 \\
\hline$\left\langle V_{\mathrm{az}}\right\rangle$ & -71 & 273 & -100 & 259 & 100 & -100 & -100 & 135 \\
\hline$\left\langle V_{\Delta E}\right\rangle$ & 416 & 128 & 520 & 302 & 250 & 520 & 470 & 122 \\
\hline$\langle v\rangle$ & 77 & 152 & 25 & 22 & 165 & 6 & 170 & 27 \\
\hline Expected in subsample & $\mathrm{s} 1, \mathrm{~s} 2, \mathrm{~s} 3$ & $\mathrm{~s} 2, \mathrm{~s} 3, \mathrm{~s} 4$ & $\mathrm{~s} 2, \mathrm{~s} 3$ & $\mathrm{~s} 3, \mathrm{~s} 4$ & unknown & unknown & unknown & unknown \\
\hline
\end{tabular}

Notes. All velocities are given in $\mathrm{km} \mathrm{s}^{-1}$ and the $\nu$-angles in ${ }^{\circ}$. Note that " $\mathrm{S}_{2}$ " is probably the " $\omega$ Cen" stream.

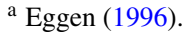

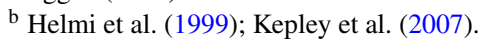

${ }^{\mathrm{c}}$ Dinescu (2002); Brook et al. (2004).

${ }^{\mathrm{d}}$ Re Fiorentin et al. (2005).

e Dettbarn et al. (2007).

${ }^{\mathrm{f}}$ Paper I.

we adopt is at the lower end of values given in the literature; for example, Soubiran et al. (2003) found $\langle\theta\rangle=159 \pm 5 \mathrm{~km}$ $\mathrm{s}^{-1}$ from spectroscopic and kinematical analyses of nearly 400 , mostly clump-giant, stars. However, their sample was limited to stars with abundances $[\mathrm{Fe} / \mathrm{H}]>-0.65$ and distances $d \lesssim$ $800 \mathrm{pc}$, so the Chiba \& Beers (2000) data more closely resemble our own data. It should also be noted, for the purpose of this exercise, that we have not explicitly included the possible presence of stars from an outer-halo component. As Carollo et al. (2007) have argued, such stars only begin to dominate 15$20 \mathrm{kpc}$ from the Galactic center and are not likely to comprise a major component in our solar neighborhood sample. 


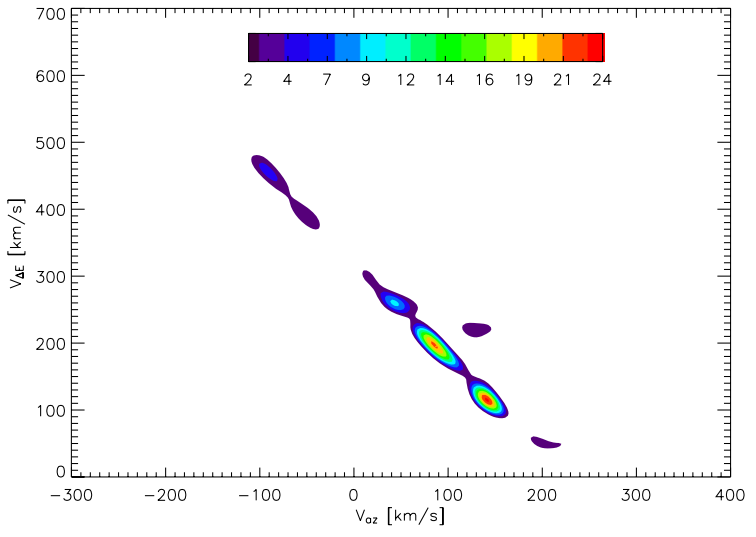

(a) without additional distance errors

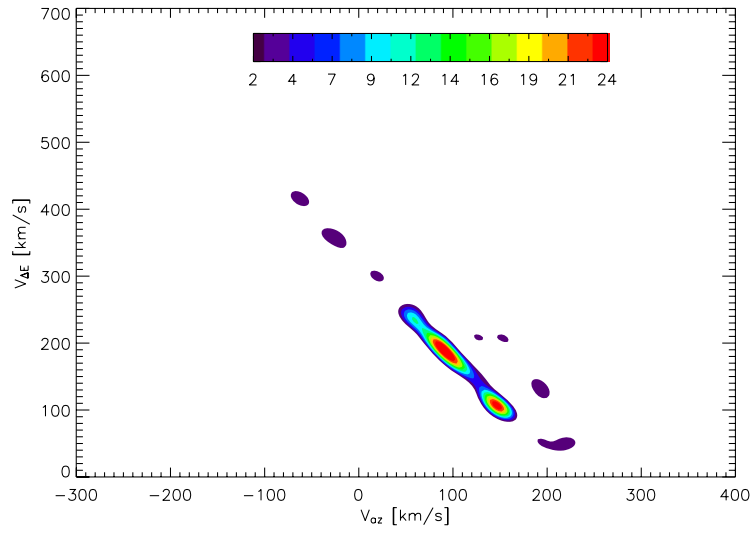

(b) with additional $-10 \%$ distance errors

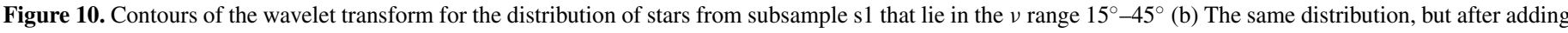

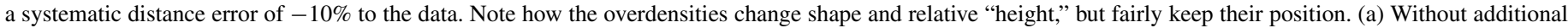
distance errors and (b) with additional $-10 \%$ distance errors.

To each velocity drawn from the smooth kinematic models (with Gaussian velocity ellipsoids) we assigned as an additional velocity error the mean velocity error of our data, that is, $(\langle\Delta U\rangle,\langle\Delta V\rangle,\langle\Delta W\rangle)=(14.9,15.7,12.2) \mathrm{km} \mathrm{s}^{-1}$. For each metallicity subsample we built 30 Monte Carlo realizations with (by construction) smooth velocity distributions, consisting of the same number of stars as the subsample. The Monte Carlo samples were then analyzed in the same way as the real data, that is, by collecting the stars in different $v$-bins and performing the wavelet analyses in these bins. Because the number of stars in each $v$-bin is small, we expect a considerable amount of shot noise. Also, because the velocity dispersions are very large, small deviations from our choice of the velocity ellipsoid would probably result in large changes of the significance levels. In addition, a stellar stream populating a certain part of phase space with stars will increase the level of Poissonian fluctuations, which are proportional to $\sqrt{N}$. When we divide the residuals between our smooth model and these fluctuations by the expected sigma for the smooth model, it may lead to the appearance of apparently highly significant multiple, adjacent, peaks (while in fact they are a part of the same structure). We therefore treat the significances with care and keep in mind that one single stream can produce multiple close features. Figure 11 shows, as an example, the significance map for subsample s3, where we have only displayed areas with $\sigma \geqslant 2$. We inspected the significance maps for the other subsamples as well and derived significance levels for all putative stellar streams (for more details, see Klement 2009). Individual results are discussed in the following section.

\section{THE RESULTS: STELLAR HALO STREAMS}

Here we explore the best "slicing" in orbital parameter- and metallicity-space by using $\left(V_{\mathrm{az}}, V_{\Delta E}, \nu,[\mathrm{Fe} / \mathrm{H}]\right)$. Metallicity is an additional constraint to distinguish tidal and dynamical streams or different streams that occupy the same region in $\left(V_{\mathrm{az}}, V_{\Delta E}, v\right)$ space. Tidal debris still carries the chemical information of its progenitor, while dynamical streams are composed of stars that lack a common origin. In addition, the latter will contribute to our sample to a lesser extent as the metallicity decreases, because dynamical streams are dominated by disk stars. In the following, we will discuss all the "phasespace overdensities" that we identified either as previously known streams or likely candidates for new stellar halo streams.
Table 2

Main Characteristics of the Stellar Streams Considered Real

\begin{tabular}{|c|c|c|c|c|c|c|c|c|}
\hline Stream & References & $\begin{array}{c}\mathrm{V}_{\mathrm{az}} \\
\left(\mathrm{km} \mathrm{s}^{-1}\right)\end{array}$ & $\begin{array}{l}\mathrm{V}_{\Delta E} \\
\left.\mathrm{~km} \mathrm{~s}^{-1}\right)\end{array}$ & $\begin{array}{c}v \\
\left(^{\circ}\right)\end{array}$ & $\sigma$ & \multicolumn{2}{|c|}{$N\langle[\mathrm{Fe} / \mathrm{H}]\rangle$} & $\sigma_{[\mathrm{Fe} / \mathrm{H}]}$ \\
\hline H99 & Helmi et al. (1999) & 300 & 120 & 150 & 12.0 & 21 & -1.8 & 0.4 \\
\hline RAVE & Klement et al. (2008) & 120 & 150 & 30 & 3.0 & 19 & -1.4 & 0.3 \\
\hline $\mathrm{S}_{3}$ & Dettbarn et al. (2007) & -100 & 470 & 155 & 4.5 & 33 & -1.6 & 0.4 \\
\hline $\mathrm{C} 1$ & new & -60 & 410 & 15 & 2.9 & 32 & -1.5 & 0.2 \\
\hline $\mathrm{C} 2$ & new & -100 & 470 & 135 & 3.4 & 53 & -1.6 & 0.4 \\
\hline $\mathrm{C} 3$ & new & -130 & 510 & 170 & 3.7 & 44 & -1.7 & 0.4 \\
\hline $\mathrm{C} 4$ & new & 175 & 75 & 100 & 4.8 & 20 & -2.3 & 0.3 \\
\hline
\end{tabular}

Notes. The parameter $\sigma$ denotes the significance of the stream obtained as described in Section 6, $N$ is the number of putative stream members, $\langle[\mathrm{Fe} / \mathrm{H}]\rangle$ is the mean metallicity of the stream, and $\sigma_{[\mathrm{Fe} / \mathrm{H}]}$ is the standard deviation.

The preconditions for selecting these stream candidates included a sufficiently large number of stars $(N>15)$, a significance level $\sigma \geqslant 2$, and a metallicity distribution consistent with a tidal origin. Table 2 summarizes the main properties of the identified streams. Lists of the putative stream members, their stellar parameters, and their derived distances and kinematic parameters are provided in the appendix. We first consider any smooth components in the phase-space distribution and then concentrate on putative halo stars on non-disk-like orbits.

\subsection{The Smooth Thick-Disk Component}

We now discuss the phase-space distribution of the stars, starting with the highest metallicity bin, s1 (Figure 6). Stars in this metallicity range, between $[\mathrm{Fe} / \mathrm{H}]=-0.5$ and -1.0 , should be dominated by members of the thick disk; this is clearly discernible as the smooth distribution of stars with polar angles around $90^{\circ}$ that rotate with $\left\langle V_{\mathrm{az}}\right\rangle \approx 200 \mathrm{~km} \mathrm{~s}^{-1}$ around the Galactic center. In the significance map, we have also found a hint of the "Hercules" stream at $\left(V_{\mathrm{az}}, V_{\Delta E}\right) \approx(169,73) \mathrm{km} \mathrm{s}^{-1}$.

For more highly inclined orbits, the distribution of stars peaks at lower azimuthal velocities (see Figure 6), indicating that the fraction of halo stars increases. In addition, at angles of $\left|v-90^{\circ}\right| \gtrsim 45^{\circ}$, overdensities of stars on retrograde orbits begin to emerge. There is no longer a smooth stellar component on such orbits. At these orbital inclinations, a few stars are sufficient to create an artificially high signal, because the variances of the residuals between the single Monte Carlo realizations and their 


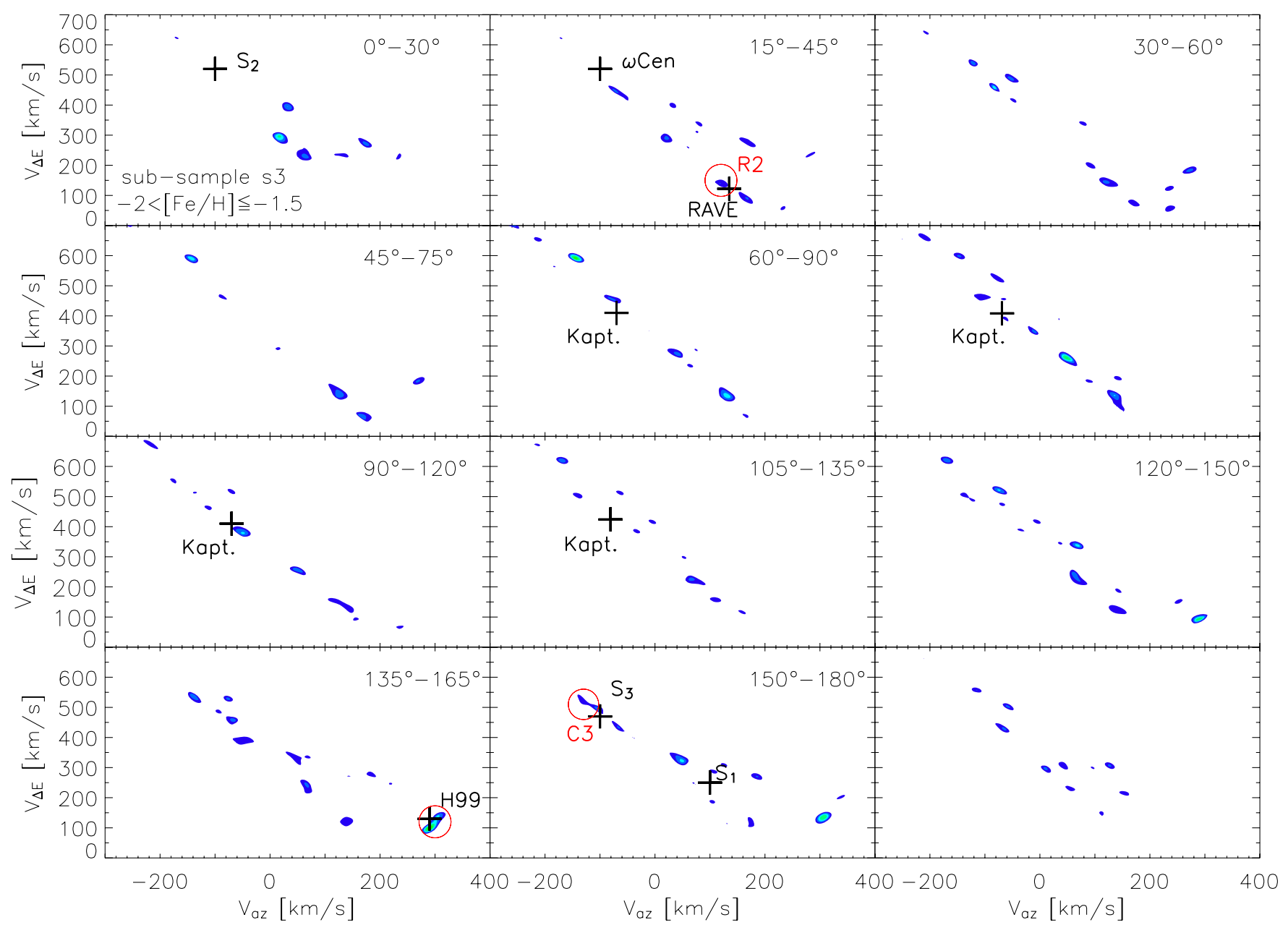

Figure 11. Significance map of the overdensities from Figure 8. Only areas with $\sigma \geqslant 2$ are shown. The contours range from 2 (blue) to 10 (red). Note the abundance of stars on disklike orbits. The crosses mark the expected position of already known stellar streams. Kapteyn's stream appears more than once, because it is well defined in $(U, V)$, but not $W$, so it spans a broader range of orbital inclinations (because $v=\arctan \frac{V}{W}$ ).

smooth superposition are nominally very small. Therefore, we question whether such overdensities in s1 are real, unless they also show up in the other metallicity subsamples.

The amount of substructure increases for the subsample s2, which consists of stars in the metallicity range $-1.5<$ $[\mathrm{Fe} / \mathrm{H}] \leqslant-1.0$ (Figure 7). There are still a large numbers of stars on disklike orbits, with $46 \%$ of the stars having orbital inclinations between $75^{\circ}$ and $105^{\circ}$. The mean rotational lag with respect to the LSR increases further. According to Chiba \& Beers (2000), $\langle\theta\rangle$ decreases linearly with $[\mathrm{Fe} / \mathrm{H}]$ for $[\mathrm{Fe} / \mathrm{H}]$ $\gtrsim-1.7$ and stays approximately constant below $[\mathrm{Fe} / \mathrm{H}]=$ -1.7 .

It is hard to make out substructure among the stars on disklike orbits, but we think we see a hint of the stream "AF06" detected by Arifyanto \& Fuchs (2006) and Helmi et al. (2006). Its stars have orbital inclinations ranging from slightly below to slightly above the Galactic plane, depending on whether we take stars with positive or negative $W$ velocities from the list of member stars given in Arifyanto \& Fuchs (2006). We detect a signal of this stream at $\left(V_{\mathrm{az}}, V_{\Delta E}\right) \approx(140,110) \mathrm{km} \mathrm{s}^{-1}$ in the $v$-slice $90^{\circ}-120^{\circ}$, maybe even extending toward $105^{\circ}-135^{\circ}$.

In the subsample $\mathrm{s} 3$, at metallicities in the range $-2.0<$ $[\mathrm{Fe} / \mathrm{H}] \leqslant-1.5$ (Figure 8 ), the fraction of halo stars dominates over thick-disk stars. In the regions of the dynamical streams "Hercules" and "AF06," we detect signals that are significant above $\sigma \geqslant 2$. These signals remain even for the subsample s4 (with $[\mathrm{Fe} / \mathrm{H}]<-2.0$ ), where the fraction of canonical thickdisk stars should be negligible, showing that still some (metalweak) thick-disk stars are present.

\subsection{Confirming the Discovery of the RAVE DR-1 Stream}

We now consider the location of the stream discovered in the RAVE DR-1 data discussed in Paper I. This stream is centered at a mean of $\left(V_{\mathrm{az}}, V_{\Delta E}\right) \approx(135,122) \mathrm{km} \mathrm{s}^{-1}$ and $v \approx 30^{\circ} .{ }^{18}$ The stars of this stream possess high vertical and low radial velocity components, $\langle W\rangle=121 \pm 2 \mathrm{~km} \mathrm{~s}^{-1}$ and $\langle U\rangle=24 \pm 2$ $\mathrm{km} \mathrm{s}^{-1}$ (Paper I, Section 6). Their $V$-velocities range from $-180 \mathrm{~km} \mathrm{~s}^{-1} \lesssim V \lesssim-140 \mathrm{~km} \mathrm{~s}^{-1}$, so their orbital plane is inclined at an angle of $v \approx 30^{\circ}$. Indeed, in the $v$-range $15^{\circ}-$ $45^{\circ}$ there exist overdensities very close to the predicted position of the "RAVE" stream: one in subsample s1, at $V_{\mathrm{az}} \approx 140 \mathrm{~km}$ $\mathrm{s}^{-1}$, which we labeled as "R 1 " (Figure 6), and one in subsamples s2 and s3, at lower azimuthal velocities of $V_{\mathrm{az}} \approx 120 \mathrm{~km} \mathrm{~s}^{-1}$, which we labeled as "R2" (Figures 7 and 8). The fact that "R2" is located at the same position in s2 and s3 makes it unlikely that it

\footnotetext{
18 In the analysis of the RAVE stars, Klement et al. (2009) projected their azimuthal motions onto the Galactic plane by adopting a cylindrical coordinate system and setting $V_{\mathrm{az}}=V, V_{\Delta E}=\sqrt{U^{2}+2\left(V_{\mathrm{az}}-V_{\mathrm{LSR}}\right)^{2}}$. This worked because the RAVE sample did not contain many halo stars. The elongation of the stream in the RAVE sample, however, could be a hint that actually more than one stream on different orbital planes has been projected onto the Galactic midplane.
} 


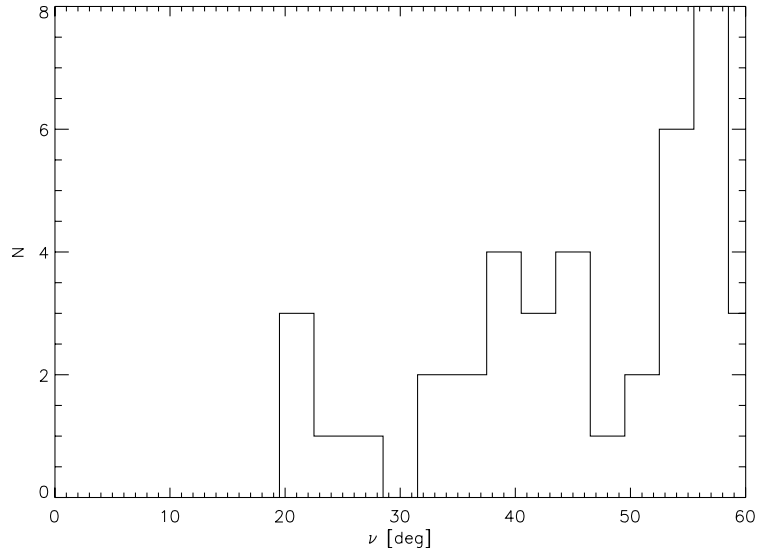

(a) 'R1'

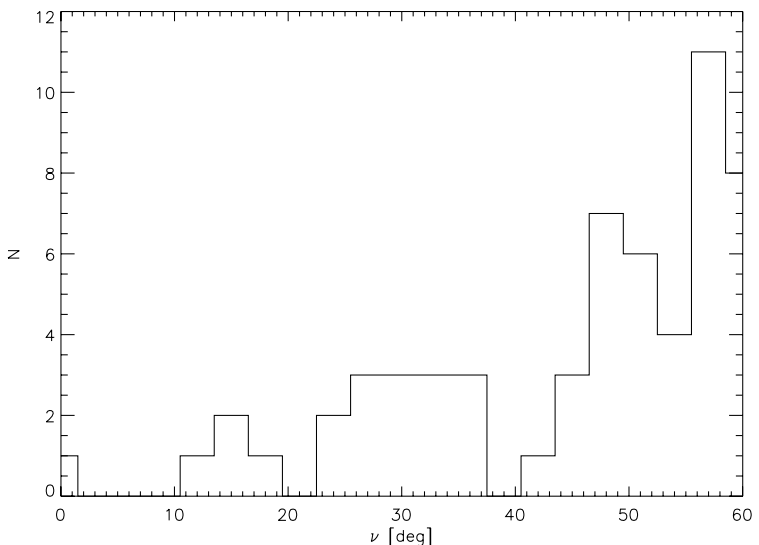

(b) 'R2'

Figure 12. Distribution of orbital inclinations $v$ of stars that lie at the same $\left(V_{\mathrm{az}}, V_{\Delta E}\right)$-position as the red contoured peaks of the overdensities "R1" in Figure 6(a) and "R2" in Figure 7(b). The distribution for "R1" is not clearly peaked around a distinct orbital polar angle, while "R2" is centered at $v \approx 30^{\circ}$. The $v$ range of $0^{\circ}-60^{\circ}$ is part of the preliminary condition for putative member stars of "R 1 " and "R2."

arises from Poisson noise. The possibility exists that "R1" and "R2," which possess slightly different angular momenta, are causally connected, perhaps resembling two distinct streams from a disrupted satellite.

To test this hypothesis, we proceed as follows. We first examine the distribution of $v$-angles for all stars that lie at the positions of "R1" and "R2" in $\left(V_{\mathrm{az}}, V_{\Delta E}\right)$ space, in order to check which orbital inclinations can be assigned to the overdensities, and if they differ for "R1" and "R2." Then we can pick stream member stars according to their positions in $V_{\mathrm{az}}, V_{\Delta E}$, and $v$, and look at their metallicity distributions.

We select the $\left(V_{\mathrm{az}}, V_{\Delta E}\right)$ position of the two streams in the following manner. For the stream at $V_{\mathrm{az}} \approx 140 \mathrm{~km} \mathrm{~s}^{-1}$, "R1," we require $\left|\left(V_{\mathrm{az}}, V_{\Delta E}\right)-(140,120)\right| \leqslant(30,30) \mathrm{km}$ $\mathrm{s}^{-1},\left|v-30^{\circ}\right| \leqslant 30^{\circ}$, and a value of the wavelet transform in s1 of at least $90 \%$ of its maximum value in that $v$ range (which corresponds to the red-colored contours in Figures 69). We also add stars from subsamples s2 and s3 that lie in this region of $\left(V_{\mathrm{az}}, V_{\Delta E}\right)$ space as possible stream members at lower metallicities, although their signature is not visible in the significance maps. For the stream "R2," at $V_{\mathrm{az}} \approx 120 \mathrm{~km}$ $\mathrm{s}^{-1}$, we require $\left|\left(V_{\mathrm{az}}, V_{\Delta E}\right)-(120,150)\right| \leqslant(30,30) \mathrm{km} \mathrm{s}^{-1}$, $\left|v-30^{\circ}\right| \leqslant 30^{\circ}$, and a value of the wavelet transform in $\mathrm{s} 2$ of at least $90 \%$ of its maximum value in that $v$ range. We also add stars from $\mathrm{s} 1$ that lie at this position as possible stream members of higher metallicity. The distribution of $v$-angles is shown in Figure 12

From inspection of Figure 12, we cannot clearly assign an orbital polar angle to the stream "R1"; stars distributed around both $v \approx 25^{\circ}$ and $v \approx 40^{\circ}$ contribute to the signal in the wavelet transform in Figure 6. In contrast, at the position of "R2" there is a group of stars that clump around $v=30^{\circ}$, which is the polar angle of the "RAVE" stream. Assuming that "R1" and "R2" stem from the same progenitor, it is possible that the difference in the $v$-distributions is caused by accelerations and decelerations of the stream stars by their precursor object's potential (Choi et al. 2007). However, in this case they still should exhibit the same metallicity distributions. The fact that "R 1 " is mostly present in the subsample s1, while "R2" shows up in s2 and s3, suggests that the $[\mathrm{Fe} / \mathrm{H}]$ distributions are different. Figure 13 compares the $[\mathrm{Fe} / \mathrm{H}]$ distributions, where we now confine the putative stream members into the $v$ range $\left|v-30^{\circ}\right| \leqslant 15^{\circ}$. In the large panels, the stars are further selected to lie at the position where the wavelet transform takes on at least $90 \%$ of its maximum value in this $v$ range, while we lessen this requirement to $75 \%$ in the small panels to obtain more stars.

The two $[\mathrm{Fe} / \mathrm{H}]$ distributions are not compatible with the hypothesis that both streams originate from the same precursor object. The $[\mathrm{Fe} / \mathrm{H}]$ distribution of "R2" peaks at metallicities between -1.2 and -1.8 , and the stream does not seem to contain stars more metal poor than $[\mathrm{Fe} / \mathrm{H}]=-0.5$. On the other hand, "R1" has one peak at $[\mathrm{Fe} / \mathrm{H}] \approx-1.6$, and a broad plateau possibly continuing beyond $[\mathrm{Fe} / \mathrm{H}]=-0.5$. We have checked for a correlation between the double-peaked $v$ distribution and the $[\mathrm{Fe} / \mathrm{H}]$ distribution of "R1," but it does not exist. We conclude from the $v$ - and $[\mathrm{Fe} / \mathrm{H}]$ distributions that a tidal origin of "R1" is ruled out. "R1" and "R2" are not correlated, in the sense that they originate from a single progenitor, and the high significance of "R1" $(\sigma \approx 8)$ could be a result of the small variance of our smooth reference model in this region of $\left(V_{\mathrm{az}}, V_{\Delta E}, v\right)$ space.

We retain "R2" as a likely tidal stream candidate and show its $(U, V, W)$ distribution in Figure 14. Here, light blue dots represent stars in the metallicity range $-1.0<\mathrm{Fe} / \mathrm{H} \leqslant-0.5$, blue dots for stars with $-1.5<\mathrm{Fe} / \mathrm{H} \leqslant-1.0$, while green dots correspond to stars in the range $-2.0<\mathrm{Fe} / \mathrm{H} \leqslant-1.5$. The small black dots are stars in the range $-2.0<\mathrm{Fe} / \mathrm{H} \leqslant-0.5$ and $\left|v-30^{\circ}\right| \leqslant 15^{\circ}$, and are displayed as the background population. The stars approximately show a "banana" shaped distribution in $(U, V)$, which is typical for tidal streams near their apocenters (Helmi et al. 2006). The banana shape results from the condition $V_{\Delta E}=$ constant, which describes an ellipse in $\left(U, V_{\mathrm{az}}\right)$, that is, in the radial and azimuthal velocities in the orbital plane of the stream. If the distribution in $W$ is sufficiently narrow, this shape also appears in the $(U, V)$ distribution.

The $U, V$, and $W$ velocities are consistent with those found in the RAVE sample: $(\langle U\rangle,\langle V\rangle,\langle W\rangle)=(-5 \pm 13,59 \pm$ $5,98 \pm 3) \mathrm{km} \mathrm{s}^{-1}$. We have good reason to believe that we have rediscovered their proposed new stream. The metallicity distribution, shown in Figure 13(b), suggests that the stream consists of stars mainly in the range $-1.8 \lesssim[\mathrm{Fe} / \mathrm{H}] \lesssim-1.2$.

\subsection{A New Stream Candidate}

Centered at even lower orbital polar angles than the "RAVE" stream in subsample s2 $(-1.5<[\mathrm{Fe} / \mathrm{H}]<-1.0)$ (Figure 7), 


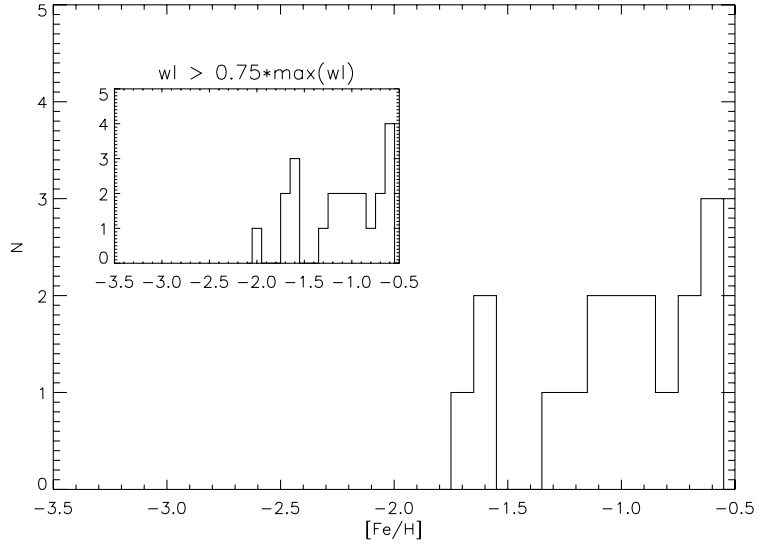

(a) 'R1'

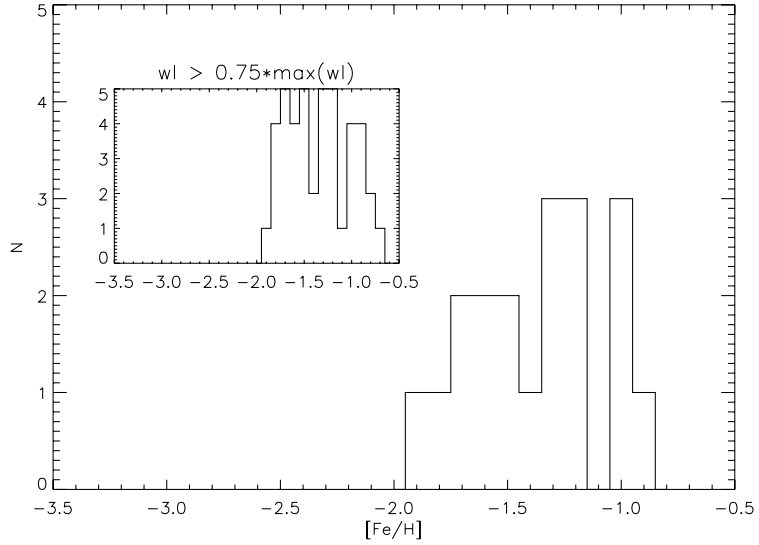

(b) 'R2'

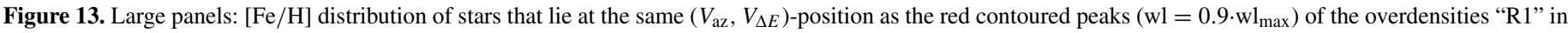

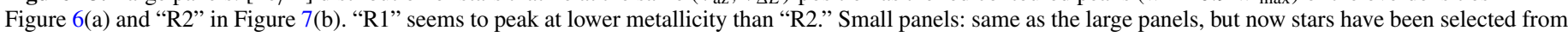
a larger region confined through $\mathrm{wl}=0.75 \cdot \mathrm{wl}_{\max }$. The difference between both $[\mathrm{Fe} / \mathrm{H}]$ distributions is more pronounced.

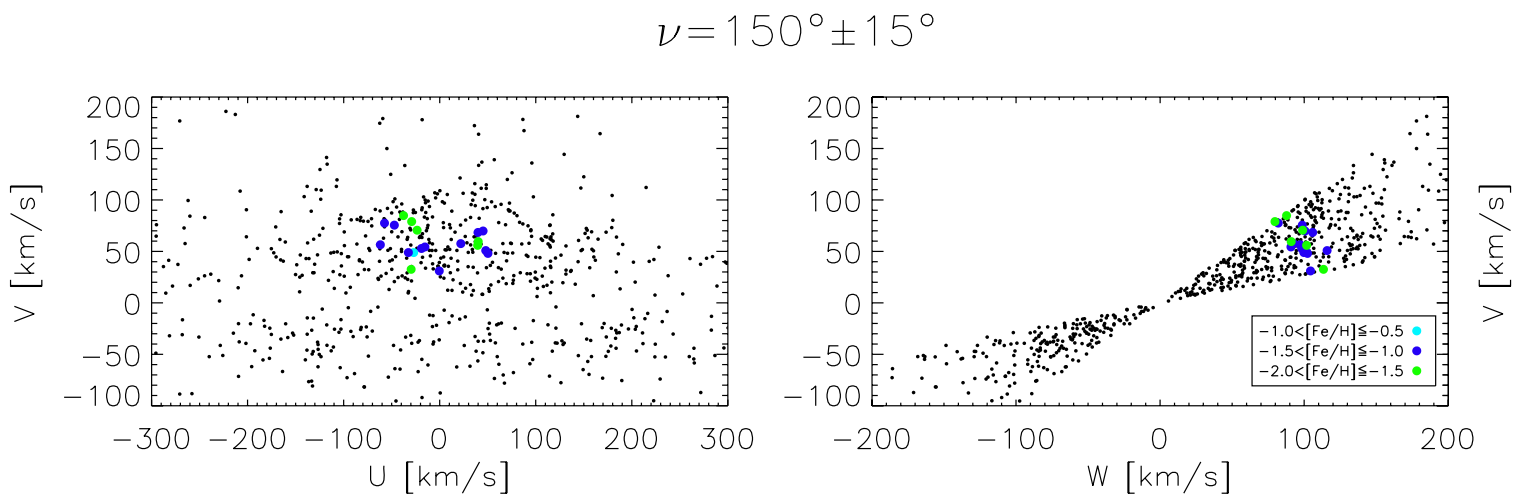

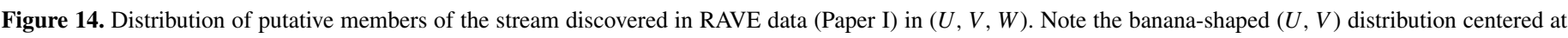
$U=0$, indicating that the stream stars are near their orbital apocenters.

(A color version of this figure is available in the online journal.)

we find an overdensity of stars around $\left(V_{\mathrm{az}}, V_{\Delta E}\right) \approx(-60,410)$. This overdensity extends toward the subsample s3, so it is unlikely to be caused by Poisson noise. Because we do not know of any stream in the literature with such kinematics, we have labeled this overdensity " $\mathrm{C} 1$ " for our first new stream candidate. The significance of this feature is $\sigma \approx 2.9$. We analyze its $[\mathrm{Fe} / \mathrm{H}]$, angular momentum, and velocity distribution in Figure 15.

Because the velocity distribution is symmetric around $U=0$, these stars must be moving toward and away from their apocenters. The typical banana shape is indicated in the $(U, V)$ distribution, but not perfectly so. Because of the high polar angle of the orbit, the banana shape in $\left(U, V_{\mathrm{az}}\right)$ (which is predicted by the condition $V_{\Delta E}=$ constant) does not perfectly translate into $(U, V)$. The metallicity distribution is roughly symmetric around $[\mathrm{Fe} / \mathrm{H}]=-1.5$ and hints toward the distribution of tidal debris from a single metal-poor progenitor. We propose $\mathrm{C} 1$ to be a newly discovered halo stream passing through the solar vicinity.

\subsection{Two Related Streams?}

The retrograde stream labeled " $\mathrm{S}_{3}$ " was discovered by Dettbarn et al. (2007) as an overdensity centered at $\left(V_{\mathrm{az}}, V_{\Delta E}, \nu\right)=\left(-100 \mathrm{~km} \mathrm{~s}^{-1}, 470 \mathrm{~km} \mathrm{~s}^{-1}, 170^{\circ}\right)$. We also find an overdensity of stars at this position in subsample s2 $(-1.5<[\mathrm{Fe} / \mathrm{H}]<-1.0)$, but peaked at $v \approx 155^{\circ}$. Never- theless, we identify this overdensity with " $\mathrm{S}_{3}$," because from Figure 3 in Dettbarn et al. (2007) we can see that the wavelet contours of their feature seem to extend toward $v \approx 155^{\circ}$.

In the $v$-slice $120^{\circ}-150^{\circ}$, we detect another overdensity at nearly the same $\left(V_{\mathrm{az}}, V_{\Delta E}\right)$ values as " $\mathrm{S}_{3}$," to which we assign the name " $\mathrm{C} 2$." This corresponds to a second peak in the $v$ distribution of stars in the $\left(V_{\mathrm{az}}, V_{\Delta E}\right)$ range centered at $\approx(-105,480) \mathrm{km} \mathrm{s}^{-1}$. We suspect that both "C2" and " $\mathrm{S}_{3}$ " contribute to the high signal of the wavelet transform in the $v$-slice $135^{\circ}-165^{\circ}$ in Figure 7 . This is confirmed in Figure 16, where we have plotted the $v$ distribution of all stars that are located at the same $\left(V_{\mathrm{az}}, V_{\Delta E}\right)$ position as the overdense region in this $v$-slice. The two peaks at $v \approx 155^{\circ}$ and $v \approx 135^{\circ}$ correspond to the streams " $\mathrm{S}_{3}$ " and " $\mathrm{C} 2$," respectively.

Figure 16 seems to suggest that " $\mathrm{S}_{3}$ " and " $\mathrm{C} 2$ " are two distinct streams that move with nearly the same orbital inclinations and eccentricities, but on different orbital planes that differ by only $\sim 20^{\circ}$. Such a double-peaked $v$ distribution could hint toward two tidal streams lost at different times from a progenitor whose orbital plane has precessed slightly during many orbits in the Milky Way. However, on a more careful look at Figure 16 we see that only a handful of stars are in each distinct peak. The apparent bimodality already becomes much less prominent when we increase the binsize of the histogram from $5^{\circ}$ to $7^{\circ}$. If we consider all sources of errors and noise $([\mathrm{Fe} / \mathrm{H}]$-determination, possible systematic distance errors, proper motion errors, unresolved 


$$
\nu=15^{\circ} \pm 15^{\circ}
$$
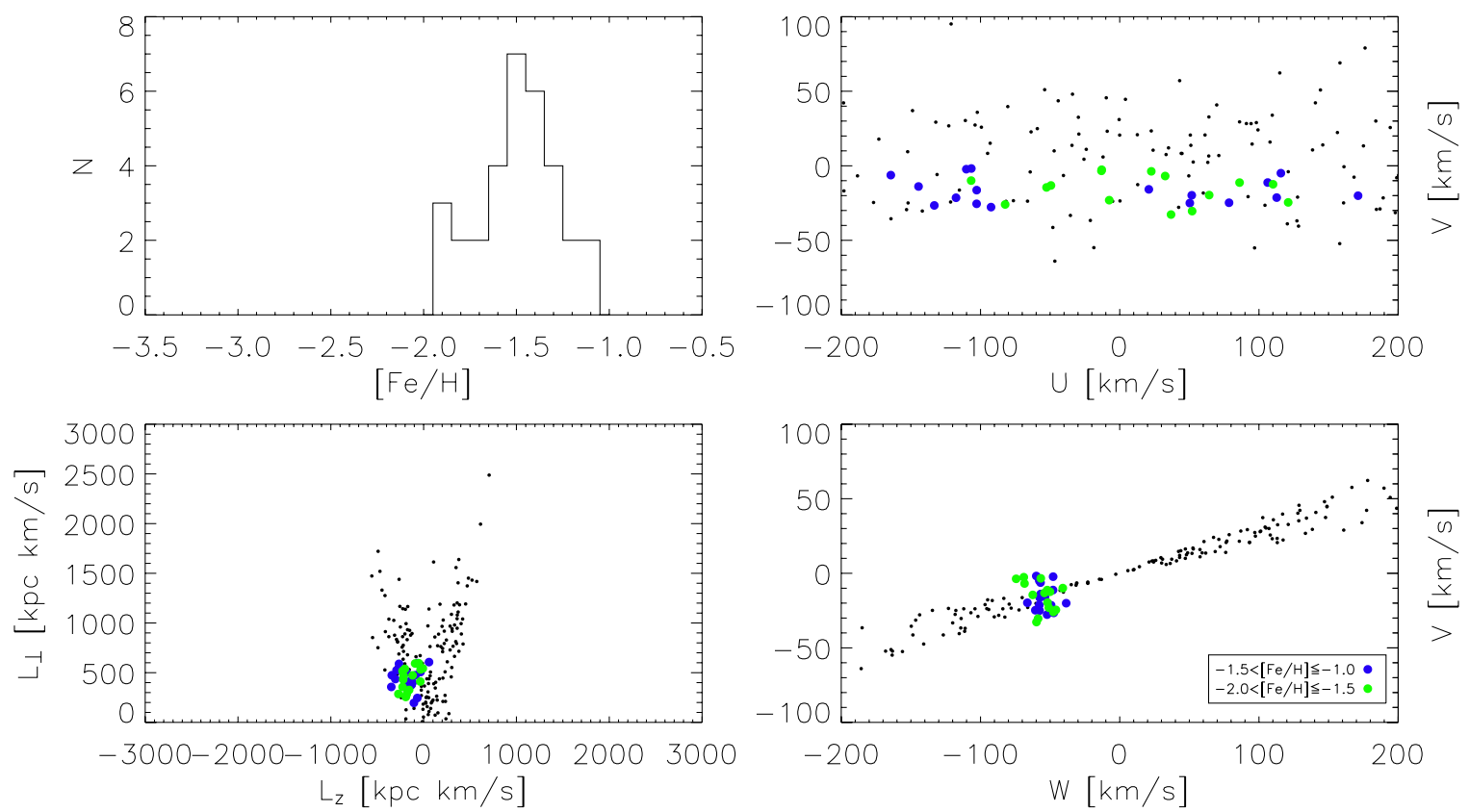

Figure 15. Distribution of members of the stream candidate " $\mathrm{C} 1$ " in metallicity $[\mathrm{Fe} / \mathrm{H}]$, angular momentum $\left(L_{z}, L_{\perp}\right)$ and $(U, V, W)$. Blue dots show stars in the range $-1.5<\mathrm{Fe} / \mathrm{H} \leqslant-1.0$, while green dots stars represent stars with $-2.0<\mathrm{Fe} / \mathrm{H} \leqslant-1.5$. The small black dots are all stars in our sample with $-2.0<\mathrm{Fe} / \mathrm{H} \leqslant-1.0$ and $\left|v-15^{\circ}\right| \leqslant 15^{\circ}$. The $(U, V)$ distribution is symmetric around $U=0$, indicating that the stream stars are well mixed and near their orbital apocenters.

(A color version of this figure is available in the online journal.)

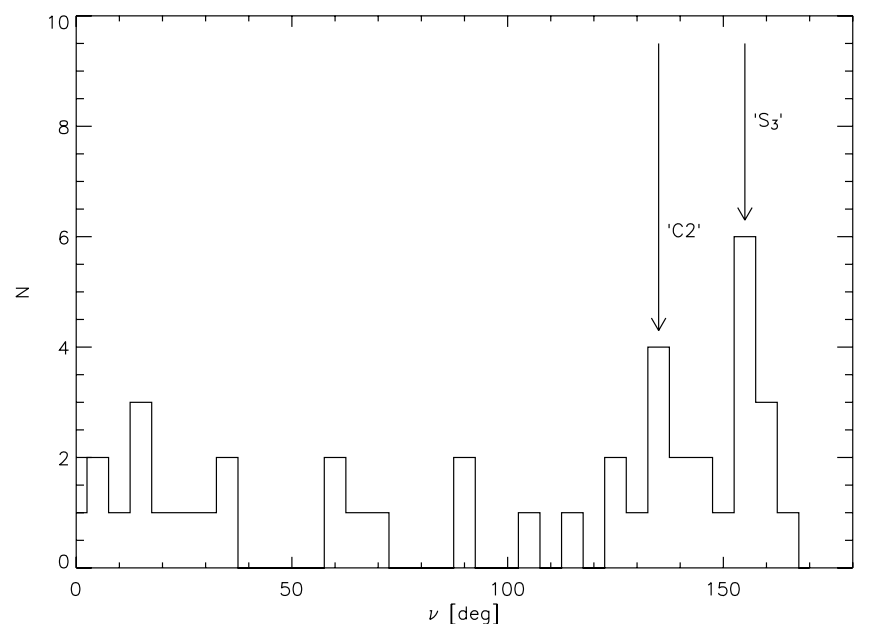

Figure 16. Distribution of orbital inclinations $v$ for all stars in subsample s2 that occupy the same region in $\left(V_{\mathrm{az}}, V_{\Delta E}\right)$ space as the overdensity labeled " $\mathrm{S}_{3}$ " in Figure $7, v$-slice $135^{\circ}-165^{\circ}$. The location of the overdensity is selected from the appropriate ranges in $V_{\mathrm{az}}$ and $V_{\Delta E}$ and the condition that the wavelet transform has at least $50 \%$ of its maximum value (to select the green-framed "bump.")

binaries, Poisson noise), could it be that we are looking at a single stream that has just been smeared out in the $v$ space by the errors?

We test this hypothesis by analyzing the kinematical and chemical properties of both features.

Figures 17 and 18 show the $[\mathrm{Fe} / \mathrm{H}],\left(L_{z}, L_{\perp}\right)$, and $(U, V, W)$ distributions for "C2" and " $\mathrm{S}_{3}$," respectively. Although it seems that "C2" does not contain stars in the range $-2.0<[\mathrm{Fe} / \mathrm{H}] \leqslant$ -1.5 from the "gap" in the wavelet transform contours in Figure 8, we include stars from subsamples s3 and s4 that lie in the same region as the putative stream members from $\mathrm{s} 2$. This is justified from the $[\mathrm{Fe} / \mathrm{H}]$ distribution shown in
Figure 17, which peaks at $[\mathrm{Fe} / \mathrm{H}] \approx-1.5$ and falls off along a tail toward lower metallicities. It resembles the distribution of a coeval tidally disrupted stellar population. We do the same for "S3," and include all stars in the range $[\mathrm{Fe} / \mathrm{H}] \leqslant-0.5$ and $\left|v-155^{\circ}\right|<15^{\circ}$. This stream also peaks around $[\mathrm{Fe} / \mathrm{H}] \approx-1.5$ (Figure 18). We note that through these selection criteria, nine stars appear in both " $\mathrm{C} 2$ " and " $\mathrm{S}_{3}$."

The banana-shaped $(U, V)$ distribution for the stream " $\mathrm{C} 2$ " indicates that its stars are near their orbital apocenters. The velocity distribution of " $\mathrm{S}_{3}$ " is only slightly different. The stars appear to be not as close to their apocenters as the "C2" stars. Both the $(U, V, W)$ and $[\mathrm{Fe} / \mathrm{H}]$ as well as the similar $L_{z}$ distributions seem to support the hypothesis that " $\mathrm{C} 2$ " and " $\mathrm{S}_{3}$ " are in fact one single stellar stream. Taking into account the typical statistical velocity errors discussed in Section 2.1.1, we find that stars at such velocities as those of "C2" could well be misplaced by $\pm 5^{\circ}$ in $v$ and $\pm 5 \mathrm{~km} \mathrm{~s}^{-1}$ in $V_{\mathrm{az}}$ and $V_{\Delta E}$, which would lead to a smearing out of one single stream in $v$ space (Figure 5.8(b); see also Klement 2009).

We conclude that there exists evidence from the $(U, V, W)$ and $[\mathrm{Fe} / \mathrm{H}]$ distributions that " $\mathrm{C} 2$ " and " $\mathrm{S}_{3}$ " belong to one single stream which might has been smeared out in $v$ space and-to a lesser degree-in $\left(V_{\mathrm{az}}, V_{\Delta E}\right)$ space. In addition, the $L_{z}$ distributions are similar enough to support this hypothesis.

\subsection{The Helmi Stream}

We again examine the metallicity range of subsample s2, $-1.5<\mathrm{Fe} / \mathrm{H} \leqslant-1.0$, and find an overdensity of stars on a highly prograde orbit inclined at $v \approx 150^{\circ}$. This is the stream discovered by Helmi et al. (1999), located at $\left(V_{\mathrm{az}}, V_{\Delta E}, v\right)=$ $\left(300,120,150^{\circ}\right)$, in very good agreement with the signal of this stream in the data set of Dettbarn et al. (2007) (see Table 1). The stream, labeled "H99," was originally discovered 
$\nu=135^{\circ} \pm 15^{\circ}$
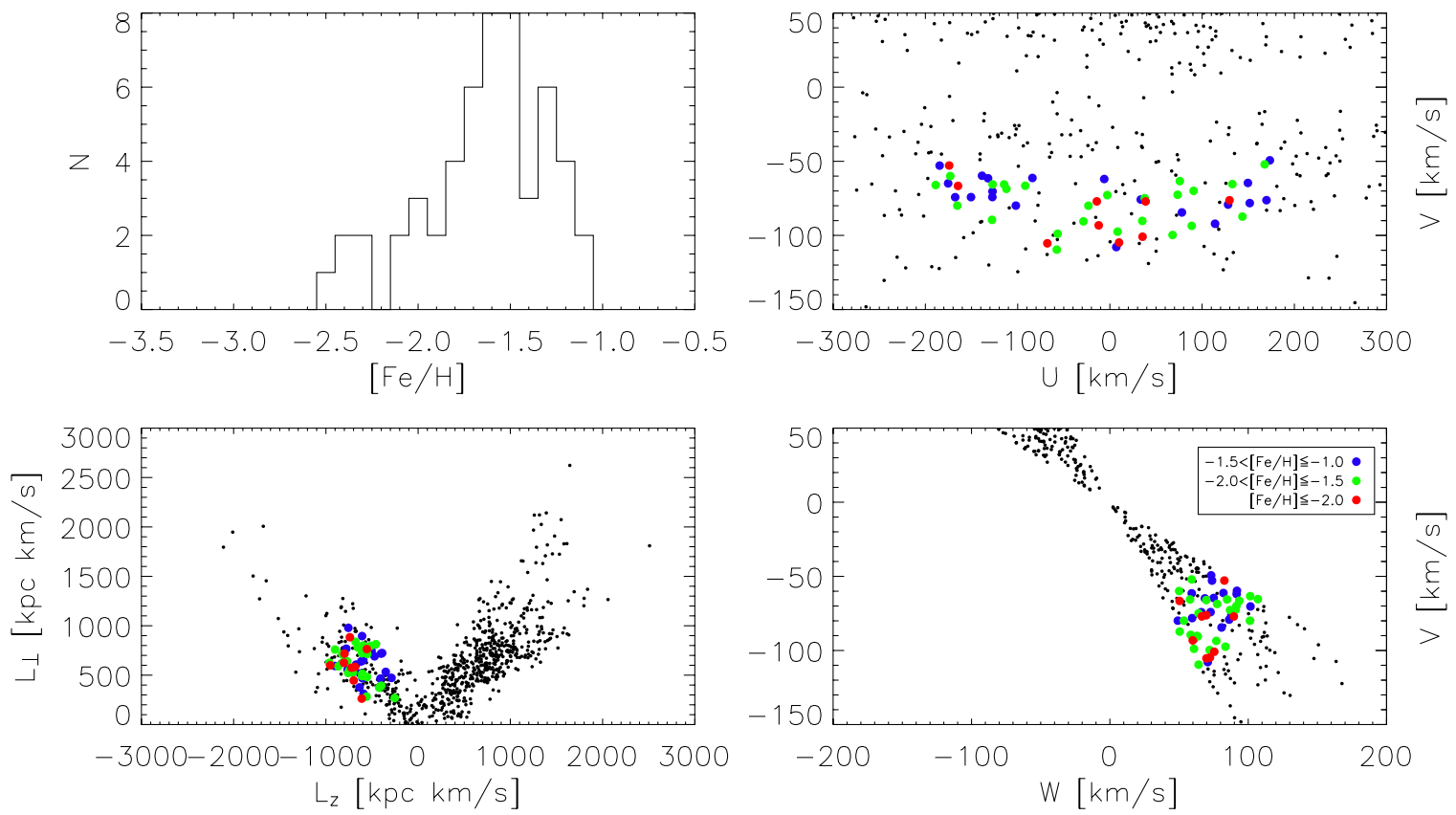

Figure 17. $[\mathrm{Fe} / \mathrm{H}],\left(L_{z}, L_{\perp}\right)$, and $(U, V, W)$ distributions for stars belonging to the stream candidate " $\mathrm{C} 2$." Light blue dots show stars in the metallicity range $-1.0<\mathrm{Fe} / \mathrm{H} \leqslant-0.5$, dark blue dots in the range $-1.5<\mathrm{Fe} / \mathrm{H} \leqslant-1.0$, and green dots show stars with $-2.0<\mathrm{Fe} / \mathrm{H} \leqslant-1.5$. The small black dots are all stars in our sample that occupy the same $[\mathrm{Fe} / \mathrm{H}]$ and $v$ ranges as the member stars of " $\mathrm{C} 2$. ."

(A color version of this figure is available in the online journal.)

$\nu=155^{\circ} \pm 15^{\circ}$
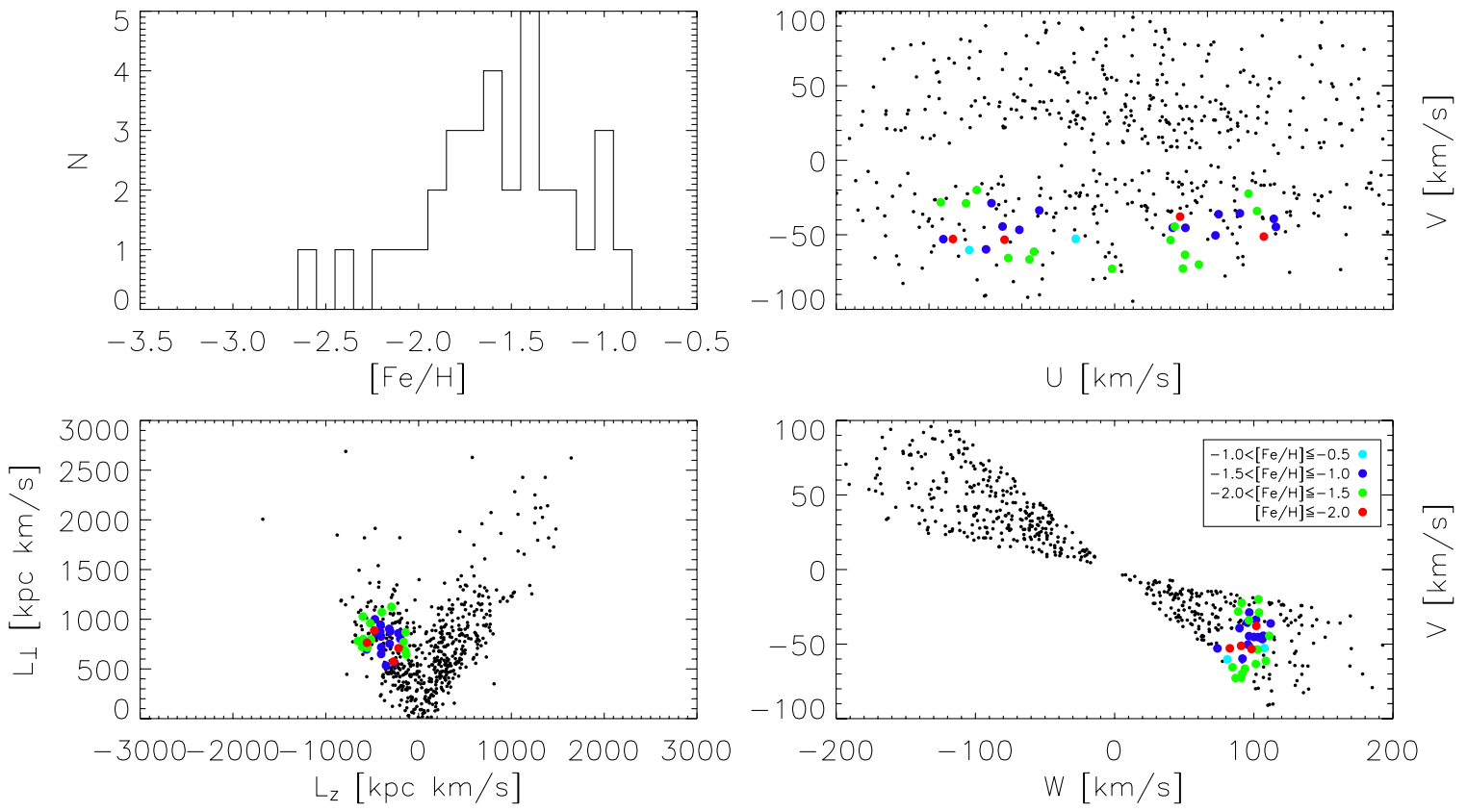

Figure 18. $[\mathrm{Fe} / \mathrm{H}],\left(L_{z}, L_{\perp}\right)$, and $(U, V, W)$ distributions for stars belonging to the stream " $\mathrm{S}_{3}$." The colors have the same meaning as in Figure 17.

(A color version of this figure is available in the online journal.)

as an overdensity of stars in the angular momentum space. Later, Chiba \& Beers (2000) confirmed the existence of this stream in their own data set and identified a possible extension toward higher azimuthal rotation. Although their data set was approximately thrice the size of the sample used by Helmi et al.
(1999), the number of stream stars $(N=10)$ stayed constant. They hypothesized that the "H99" stream could be related to the "trail" extension, if this "trail" gained angular momentum from the interaction of the progenitor with the Milky Way's gravitational potential. 


$$
\nu=150^{\circ} \pm 15^{\circ}
$$
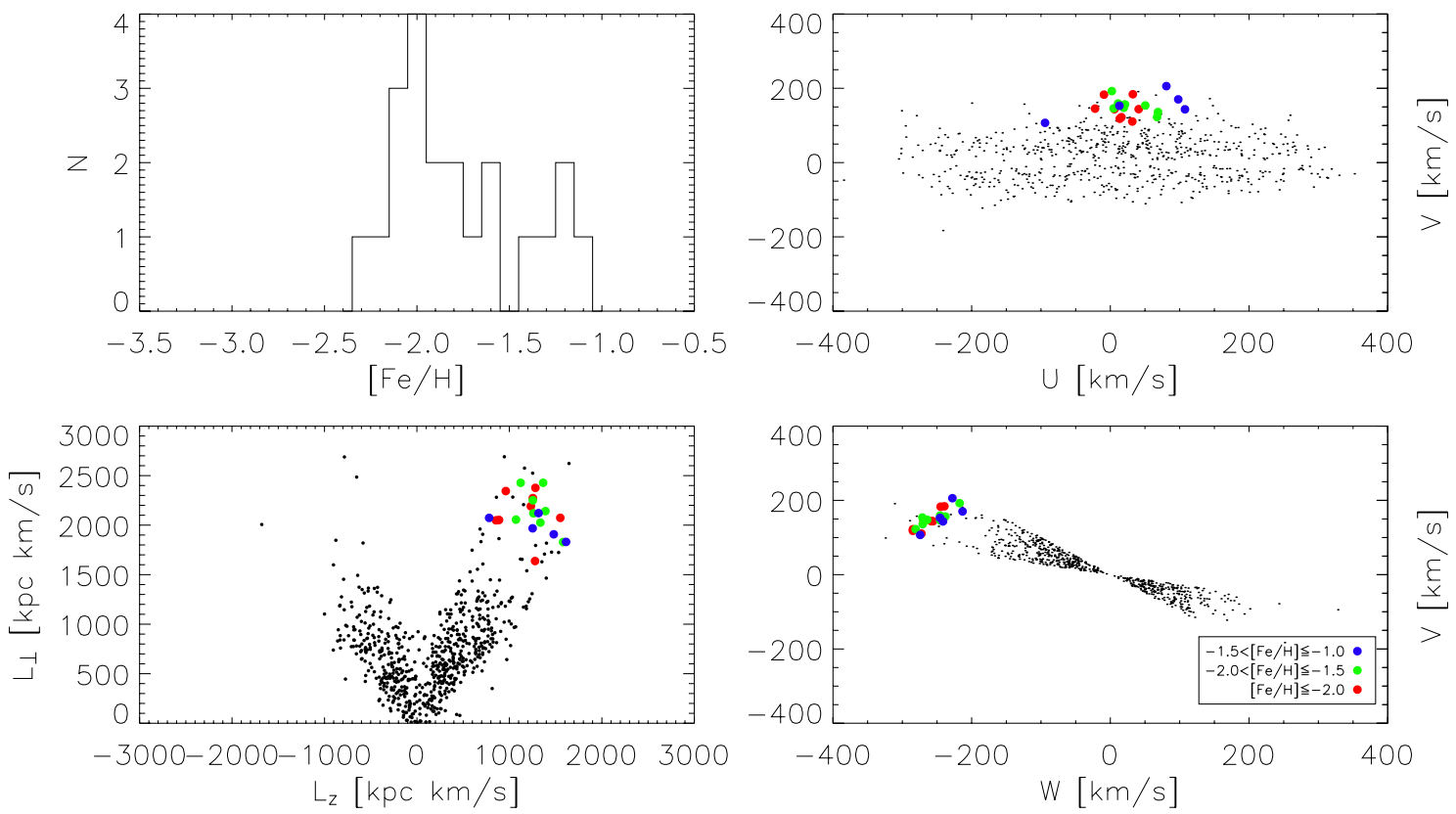

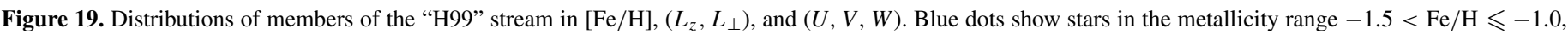

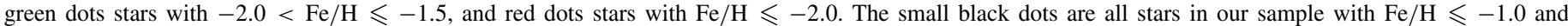
$\left|v-150^{\circ}\right| \leqslant 15^{\circ}$. The $(U, V, W)$ distribution is in very good agreement to that shown by Helmi et al. (1999, their Figure 2).

(A color version of this figure is available in the online journal.)

$$
\nu=170^{\circ} \pm 15^{\circ}
$$
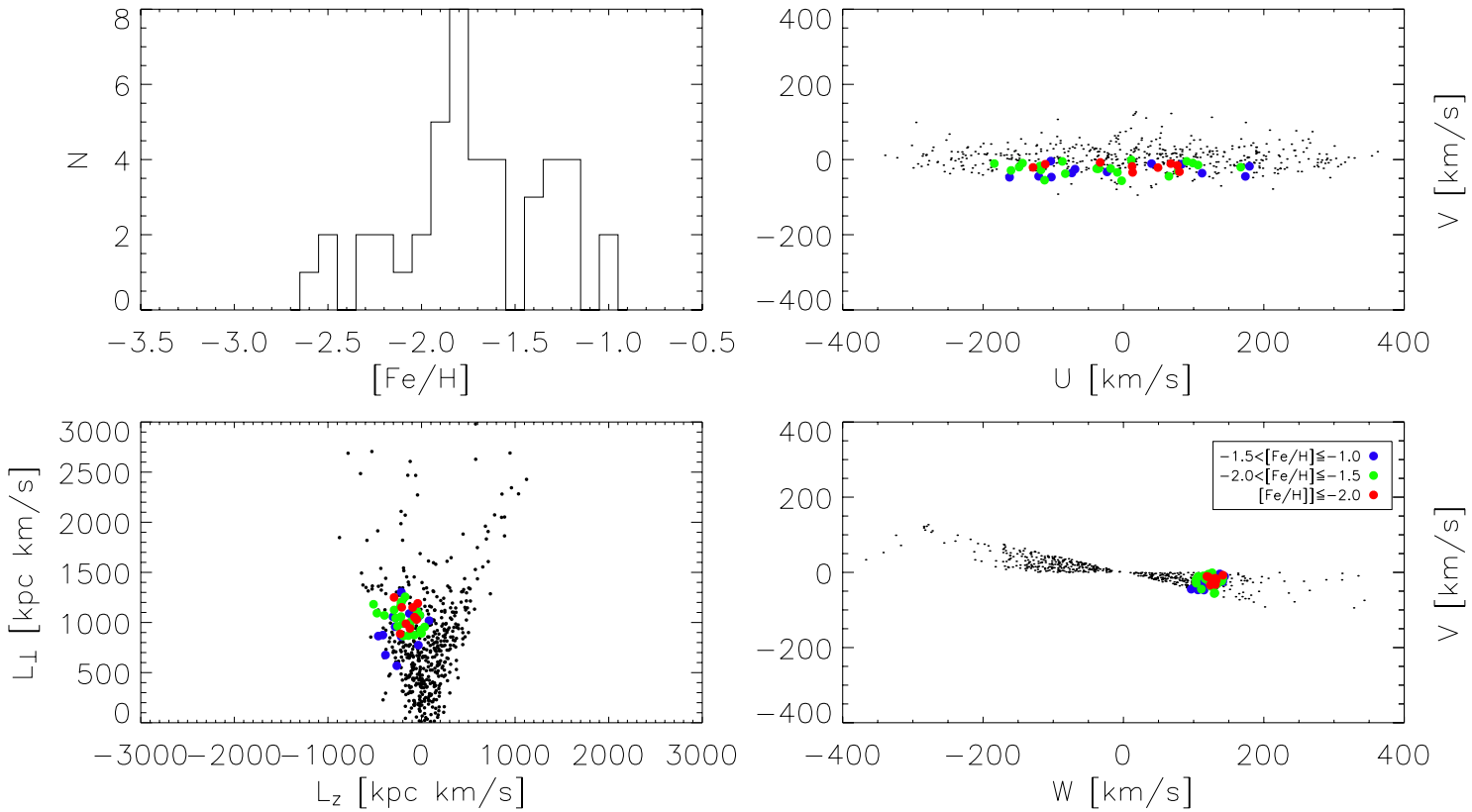

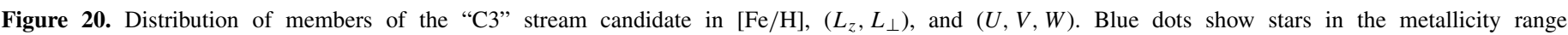

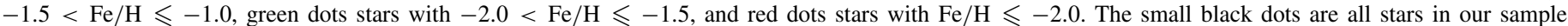
with $\mathrm{Fe} / \mathrm{H} \leqslant-1.0$ and $\left|v-170^{\circ}\right| \leqslant 15^{\circ}$.

(A color version of this figure is available in the online journal.)

Figure 19 shows the $[\mathrm{Fe} / \mathrm{H}],\left(L_{z}, L_{\perp}\right)$, and $(U, V, W)$ distributions of all stars that we assign to the "H99" stream. The stream extends toward lower metallicities and is highly significant in the subsample s4. The $(U, V, W)$ and $\left(L_{z}, L_{\perp}\right)$ distributions agree very well with those in the original work of Helmi et al. (1999, their Figure 2). The $[\mathrm{Fe} / \mathrm{H}]$ distribution peaks at $[\mathrm{Fe} / \mathrm{H}] \approx-2.0$ and does not extend beyond $[\mathrm{Fe} / \mathrm{H}] \approx-2.3$.
The range of $[\mathrm{Fe} / \mathrm{H}]$ values that we find for the "H99" stream agrees well with the $[\mathrm{Fe} / \mathrm{H}]$ values of the "H99" stream members given by Kepley et al. (2007), although they report two stream members with $[\mathrm{Fe} / \mathrm{H}]<-2.3$. The number of stars in our sample that we identify as "H99" members is $N=21$, approximately double that of previous studies. However, the number is too small to account for as much as one-tenth of the 

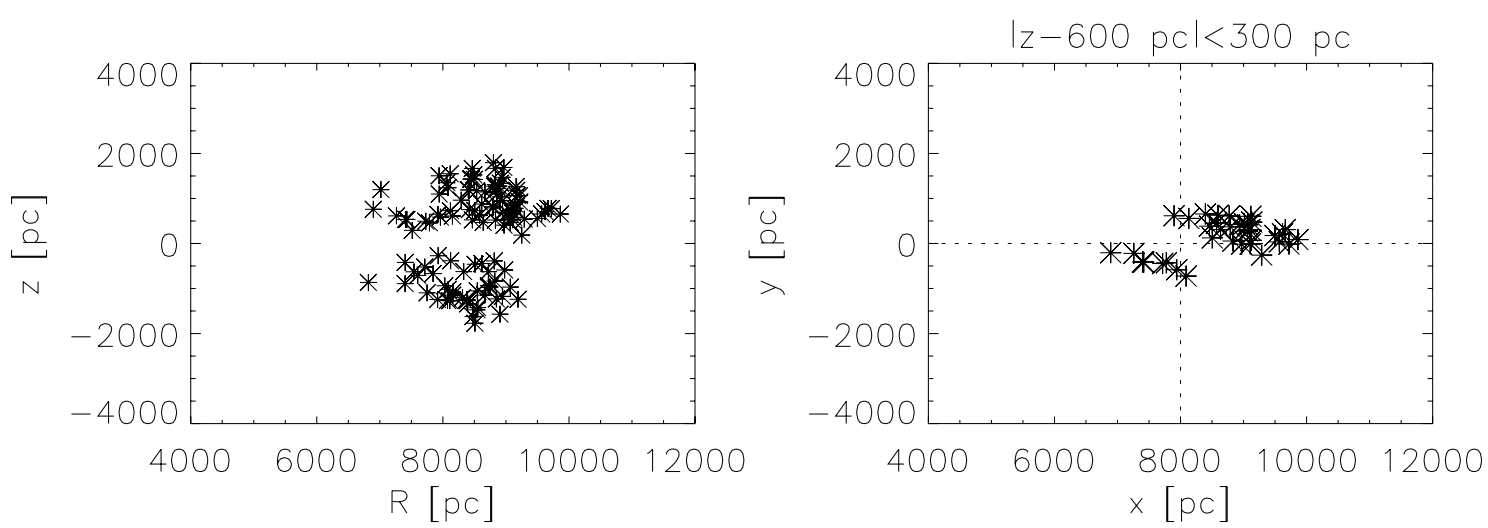

Figure 21. Spatial distribution of stars which we identify as members of the composite stream " $\mathrm{S}_{3} / \mathrm{C} 2 / \mathrm{C} 3$." In the left panel we show all 97 stars in the $(R-z)$ plane, while in the right panel we concentrate on a slice at $z=600 \mathrm{pc}$, analog to Figure 27 in Juric et al. (2008). Note the very similar location of the main overdensity in the $z=600 \mathrm{pc}$ slice.

$$
\nu=100^{\circ} \pm 15^{\circ}
$$
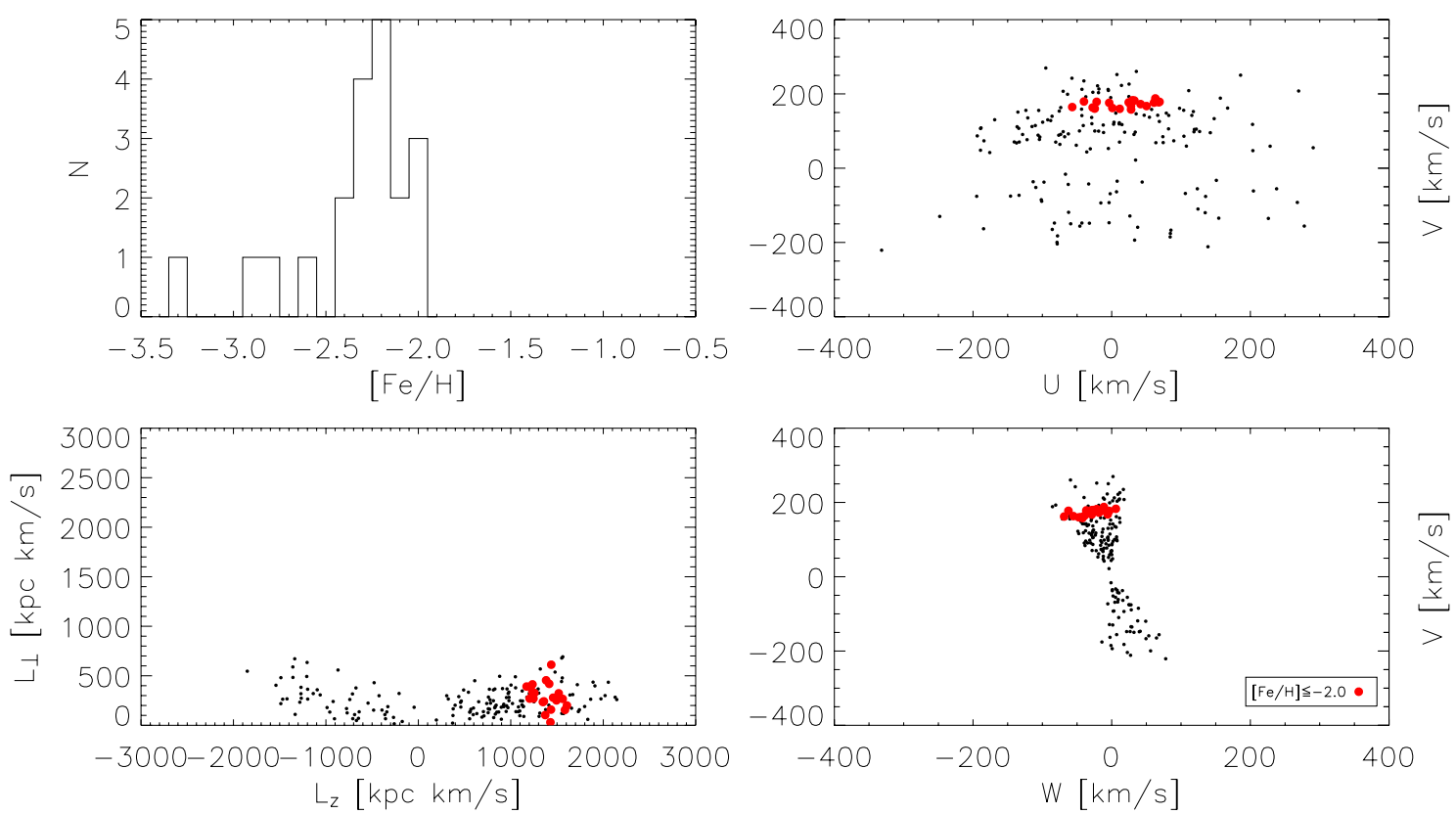

Figure 22. Distribution of members of the " $\mathrm{C} 4$ " stream candidate in metallicity $[\mathrm{Fe} / \mathrm{H}],\left(L_{z}, L_{\perp}\right)$, and $(U, V, W)$. The small black dots are all stars in our sample with $\mathrm{Fe} / \mathrm{H} \leqslant-2.0$ and $\left|v-100^{\circ}\right| \leqslant 10^{\circ}$

(A color version of this figure is available in the online journal.)

halo stars that are currently present in the solar neighborhood, as suggested by Helmi et al. (1999).

\subsection{More Substructure at Very Low Metallicities}

In the subsamples $\mathrm{s} 3(-2.0<[\mathrm{Fe} / \mathrm{H}]<-1.5)$ and $\mathrm{s} 4$ $([\mathrm{Fe} / \mathrm{H}]<-2.0)$, the amount of substructure increases further. We expect a contribution of only $\sim 10 \%$ thick-disk stars in s3 and 100\% halo stars in s4 (Chiba \& Beers 2000). While about $46 \%$ of the stars in subsample s2 move on disklike orbits $\left(75^{\circ} \leqslant v<105^{\circ}\right.$ ), this number drops to $23 \%$ for both s3 and s4. It seems that also for stars more metal poor than $[\mathrm{Fe} / \mathrm{H}]=$ -2 , a small fraction of stars with (metal-weak) thick-disk-like kinematics remains constant.

Besides the already discussed streams that are also visible in s3, "RAVE," "H99," and "C1," we find an overdensity centered at $\left(V_{\mathrm{az}}, V_{\Delta E}, v\right)=\left(-130 \mathrm{~km} \mathrm{~s}^{-1}, 510 \mathrm{~km} \mathrm{~s}^{-1}, 170^{\circ}\right)$, which we label as "C3." It is located near the stream " $\mathrm{S}_{3}$ " and-like " $\mathrm{C} 2$ ' - may be related to it. The peak in the $v$ distribution of stars in this region of $\left(V_{\mathrm{az}}, V_{\Delta E}\right)$ space at $v=170$ would fit exactly to the $v$ value originally assigned to " $\mathrm{S}_{3}$ " by Dettbarn et al. (2007). The $[\mathrm{Fe} / \mathrm{H}]$ distribution peaks at $[\mathrm{Fe} / \mathrm{H}] \approx-1.8$, and is consistent with that of " $\mathrm{S}_{3}$ " or " $\mathrm{C} 2$ " (Figure 20). The velocity and $L_{z}$ distribution of "C3" seem to differ slightly from those of the " $\mathrm{S}_{3} / \mathrm{C} 2$ " stream. However, like in the case of " $\mathrm{C} 2$ " and " $\mathrm{S}_{3}$," if we consider all possible error sources the possibility exists that one single stream manifests itself in several adjacent features. In particular, we refer to Section 5, where we have shown that a systematic distance error of $10 \%$ alone is able to cause such an effect by changing the relative "heights" of overdensities. In addition, as already noted in Section 6, the confidence levels for substructures the way we compute them depend on the priors we choose for the Monte Carlo simulations (smooth halo). But given the case that the "S $\mathrm{S}_{3} / \mathrm{C} 2$ " stream is there, its existence increases the number of stars in that region of phase space, which increases the level of Poissonian fluctuations (as they are proportional to $\sqrt{N}$ ). When we divide the residuals between a smooth background and these fluctuations by the expected 
sigma for the smooth background, it may lead to the appearance of apparently highly significant multiple, adjacent, peaks (while in fact they are a part of the same structure).

We gain confidence that " $\mathrm{C} 3$ " is a not a feature created from Poisson noise from the fact that the wavelet transform at "C3's" position in the $\left(V_{\mathrm{az}}, V_{\Delta E}\right)$ space has values greater than $90 \%$ of its maximum in both subsamples s3 and s4 (Figures 8 and 9). Furthermore, "C 3 " appears in the significance map of subsample s3 (Figure 11) at a significance level greater than 2.

Given its proximity to the " $\mathrm{S}_{3} / \mathrm{C} 2$ " stream and the uncertainties in our variables (in particular $[\mathrm{Fe} / \mathrm{H}]$, systematic distance errors, and Poisson noise), we think that "C3" is part of that same stream. Taken together, the " $\mathrm{S}_{3} / \mathrm{C} 2 / \mathrm{C} 3$ " stream consists of 97 stars whose spatial distribution shows similarities with the thick-disk overdensity found by Juric et al. (2008) at $(R, z) \approx(9.5,0.8) \mathrm{kpc}$. We show this in Figure 21, where we have adopted the coordinate system used by Juric et al. (with the $x$-axis pointing toward the Sun). A comparison to their Figure 27 (left and right panels) reveals an intriguingly similar distribution in the Northern hemisphere plus a counterpart in the Southern hemisphere which-although less prominent-is also mapped by Juric et al. in their Figure 26 (second row, right panel). The $(x, y)$-distribution in the $z=600 \mathrm{pc}$ slice reveals a main clump, whose position matches the rectangular region in the right panel of Juric et al.'s Figure 27. This is to our knowledge the first time that a six-dimensional phase-space overdensity has been first identified by its kinematics. If the similarity with the Juric et al. overdensity is not a coincidence, this would confirm this finding by an independent method. Further, the kinematics of the feature would rule out a ringlike feature of (thick) disk stars, instead supporting either a "localized clumpy overdensity" (Juric et al. 2008) or a spatially coherent stellar stream passing the plane in the northern direction. However, our survey volume is not large enough to distinguish between these two possibilities.

We note that the " $\mathrm{S}_{3} / \mathrm{C} 2 / \mathrm{C} 3$ " stream is the only stream for which we have found signs of a spatial coherence.

In the most metal-poor subsample $s 4([\mathrm{Fe} / \mathrm{H}]<-2.0)$, where the fraction of halo stars is close to $100 \%$, there exists a substantial amount of substructure; it is difficult to identify any smooth component. However, many of the overdensities in Figure 9 consist of only a few stars, and their reality is thus in doubt. We concentrate on a highly significant $(\sigma=4.8)$ feature located at $\left(V_{\mathrm{az}}, V_{\Delta E}, v\right)=\left(175,75,100^{\circ}\right)$ and labeled with "C4." In this region of $\left(V_{\mathrm{az}}, V_{\Delta E}, v,[\mathrm{Fe} / \mathrm{H}]\right)$ space, a density enhancement of stars is not expected. The " $\mathrm{C} 4$ " candidate stream is clumped around $(\nu,[\mathrm{Fe} / \mathrm{H}]) \approx\left(100^{\circ},-2.2\right)$, which clearly distinguishes it from a smooth feature. Figure 22 shows the metallicity, angular momentum and $(U, V, W)$ distribution of "C4."

The very low eccentricity, $e=V_{\Delta E} / \sqrt{2} V_{\mathrm{LSR}} \simeq 0.2$, of "C4" suggests that it belongs to a metal-weak thick disk. However, according to Chiba \& Beers (2000) even the metal-weak tail of the thick disk should not contain stars as metal poor as $[\mathrm{Fe} / \mathrm{H}]$ $\lesssim-2.2$, where the $[\mathrm{Fe} / \mathrm{H}]$ distribution of " $\mathrm{C} 4$ " peaks. These authors argued that the fraction of low-eccentricity stars with $[\mathrm{Fe} / \mathrm{H}] \leqslant-2.2$ remains the same regardless of their height $|z|$, implying that they purely belong to the halo. In addition, stars in the clump "C4" are not distributed symmetrically around $v=90^{\circ}$, but are centered at an orbital polar angle of roughly $100^{\circ}$, with a longer tail toward higher inclinations.

On the other hand, the formation of the stellar halo and thick disk might underlie a common cause-the accretion of satellite galaxies. It has been shown that the metal-weak tail of the thick disk could consist of tidal debris stemming from a progenitor on a planar orbit that has been circularized prior to disruption through dynamical friction (Quinn et al. 1986, 1993; Abadi et al. 2003). The very low abundances of the "C4" stars favor this scenario. Even if these stars do not belong to a single progenitor exclusively, we have found evidence that tidal debris exists on disklike orbits, giving further support for the hierarchical buildup of the Milky Way and making "C4" the most intriguing of our newly detected streams.

\section{CONCLUSIONS}

We have used SDSS/SEGUE data from the seventh SDSS public data release to search for halo streams in a $2 \mathrm{kpc}$ sphere centered on the Sun. Using the cataloged values for $\log \mathrm{g}, g-i$ color, and $[\mathrm{Fe} / \mathrm{H}]$, together with distance estimates based on the photometric parallax relation from Ivezić et al. (2008), we assembled a sample of 22,321 subdwarfs with $[\mathrm{Fe} / \mathrm{H}] \leqslant-0.5$, excluding main-sequence turnoff stars. A comparison with fiducial sequences for 12 globular clusters from An et al. (2008) suggests that our distances are accurate to within systematic errors of less than 5\%, although they are much less constrained on the high-metallicity end of the photometric parallax relation.

We divided our sample into four subsamples, equally spaced by 0.5 dex in metallicity. Assuming a spherical potential, we searched for stellar streams in each subsample in a space spanned by the quantities $V_{\mathrm{az}}=\sqrt{V^{2}+W^{2}}, V_{\Delta E}=$ $\sqrt{U^{2}+2\left(V_{\mathrm{az}}-V_{\mathrm{LSR}}\right)^{2}}$, and $v=\arctan \frac{V+V_{\mathrm{LSR}}}{W}$. These quantities are approximations for a star's azimuthal velocity or angular momentum, eccentricity, and orbital angle with respect to the positive $z$-axis.

Our basic results can be summarized as follows.

1. Our sample is dominated by stars on disklike orbits; the fraction of these stars with orbital inclinations between $75^{\circ}$ and $105^{\circ}$ is $81 \%(80 \%)$ for stars with $-1.0 \leqslant[\mathrm{Fe} / \mathrm{H}]<$ $-0.5,46 \%(44 \%)$ for stars with $-1.0 \leqslant[\mathrm{Fe} / \mathrm{H}]<-0.5$, and remains constant at $23 \%(16 \%-17 \%)$ for all stars more metal poor than $[\mathrm{Fe} / \mathrm{H}]=-1.5$ (the number in parenthesis gives the fraction of stars on prograde orbits). This implies that beyond $[\mathrm{Fe} / \mathrm{H}] \simeq-1.5$, the fraction of thick-disk stars remains constant.

2. In the metallicity range $-1.0 \leqslant[\mathrm{Fe} / \mathrm{H}]<-0.5$, it is difficult to identify substructure among the thick-disk stars, because the smooth component dominates. As the fraction of thick-disk stars decreases, we detect signals of the stream first described by Arifyanto \& Fuchs (2006) and Helmi et al. (2006).

3. We find an overdensity of stars moving with disklike kinematics, but too metal poor to belong to the classical metal-weak thick disk, which should not extend beyond $[\mathrm{Fe} / \mathrm{H}] \simeq-2.2$ (Chiba \& Beers 2000). We interpret this clump, named "C4," as a tidal stream accreted on an orbit in the plane of the protodisk. Halo streams on such orbits are predicted from both numerical simulations of spiral galaxy formation (Quinn et al. 1986, 1993; Abadi et al. 2003 ) and the considerable (20\%) fraction of halo stars on low-eccentricity orbits (Chiba \& Beers 2000).

4. We confirm the existence of previously detected halo streams: the "RAVE" stream, which was discovered in data from the first RAVE data release (Paper I), the stream " $\mathrm{S}_{3}$ " found by Dettbarn et al. (2007), which crosses the solar 
neighborhood from the direction of the South Galactic Pole on a diagonally retrograde orbit, and the "H99" stream discovered by Helmi et al. (1999) at high angular momentum. The latter is the most significant stream in our sample ( $\sigma \approx 12.0$ ), which explains why Helmi et al. (1999) found it in a sample of only 275 stars. However, even with our much larger sample size the number of stars that belong to this stream is only approximately doubled. This speaks against the conclusion of Helmi et al. (1999) that as much as $10 \%$ of the nearby halo stars originated from a single progenitor.

The " $\mathrm{S}_{3}$ " stream lies very close to two other features, which we labeled "C2" and "C 3 ," in $\left(V_{\mathrm{az}}, V_{\Delta E}, v\right)$. Both possess similar kinematics as " $\mathrm{S}_{3}$," and the small differences could well be explained by the effects of statistical and systematic distance errors and by the way we compute statistical significances, which may lead to adjacent peaks as a stellar stream enhances the number of stars in a certain region of the phase space. The $[\mathrm{Fe} / \mathrm{H}]$ distributions of " $\mathrm{S}_{3}$," "C2 ," and "C 3 " give strong support for the hypotheses that all three features are one single stream. The composite " $\mathrm{S}_{3} / \mathrm{C} 2 / \mathrm{C} 3$ " stream shows intriguing similarities in its spatial extent to an overdensity found by Juric et al. (2008) at $(R, z) \approx$ $(9.5,0.8) \mathrm{kpc}$. If this connection is real, this would be the first time that a six-dimensional coherent phase-space overdensity has been identified by its kinematics alone. Further, because the $W$ velocities are too high for thickdisk stars, we could rule out a ringlike feature in the thick disk as its origin.

The fact that both the "RAVE" and the "S $\mathrm{S}_{3} / \mathrm{C} 2 / \mathrm{C} 3$ " streams have been found in two independent samples makes it very unlikely that they are "false positives." The latter is significant at a level of $\sigma \gtrsim 2.8$ in our data (the " $\mathrm{S}_{3}$ " peak; Figure 11) and in the data from Dettbarn et al. (2007), corresponding to a confidence level of $99.5 \%$. In other words, the probability that this stream is created by chance in both samples is $(0.005)^{2}=0.0025 \%$. The significance levels of the "RAVE" stream in the RAVE data and in the SDSS/SEGUE data are $\sigma \gtrsim 3$ (Paper I; Figure 10) and $\sigma \approx 3.0$ (Figure 11), respectively, which also makes it very unlikely that both streams are created through Poisson noise.

5. Besides the already known features, we find evidence of a large amount of substructure, especially in the most metalpoor bins. In particular, we identify one candidate for a genuine halo stream that has not yet been described in the literature. This stream, "C1," moves on highly inclined orbits nearly in the direction toward the north Galactic Pole.

6. We can roughly estimate the fraction of halo stars contained within the streams we detected. To obtain an upper limit, we simply treat all stars as halo stars that lie outside the range $75^{\circ}<v<90^{\circ}$ and outside of $V_{\mathrm{az}}>0,[\mathrm{Fe} / \mathrm{H}]$ $>-1.0$. This results in 4388 stars, of which $53(1.2 \%)$ account for the most well populated peak in our sample, "C2." The "H99" stream, with 21 members, only represents a $0.48 \%$ maximum fraction of halo stars. These results are fully consistent with statistical arguments made by Gould (2003) considering the fraction of halo stars in a single stream. He puts an upper limit on the granularity of the halo, concluding that if the halo would be fully composed of kinematically cold stellar streams, then at $95 \%$ confidence no single stream could contain more than a $5 \%$ fraction of the halo stars.
7. Metallicities are very helpful when it comes to deciding about the origin of a moving group. Our stream candidates exhibit a $[\mathrm{Fe} / \mathrm{H}]$ distribution with a single peak, indicating that their progenitor had a well defined star-forming epoch.

This study shows the power of current and future large-scale surveys to probe substructure in the solar neighborhood and the Milky Way in general. Extensions of this technique to include additional chemical information, for example, $[\alpha / \mathrm{Fe}]$ and $[\mathrm{C} / \mathrm{Fe}]$ ratios, are being pursued at present.

We thank the anonymous referee for his very thorough report which highly improved our manuscript and contributed to some of our major findings.

Funding for the SDSS and SDSS-II has been provided by the Alfred P. Sloan Foundation, the Participating Institutions, the National Science Foundation, the U.S. Department of Energy, the National Aeronautics and Space Administration, the Japanese Monbukagakusho, the Max Planck Society, and the Higher Education Funding Council for England. The SDSS Web site is http://www.sdss.org/.

The SDSS is managed by the Astrophysical Research Consortium for the Participating Institutions. The Participating Institutions are the American Museum of Natural History, Astrophysical Institute Potsdam, University of Basel, University of Cambridge, Case Western Reserve University, University of Chicago, Drexel University, Fermilab, the Institute for Advanced Study, the Japan Participation Group, Johns Hopkins University, the Joint Institute for Nuclear Astrophysics, the Kavli Institute for Particle Astrophysics and Cosmology, the Korean Scientist Group, the Chinese Academy of Sciences (LAMOST), Los Alamos National Laboratory, the Max-PlanckInstitute for Astronomy (MPIA), the Max-Planck-Institute for Astrophysics (MPA), New Mexico State University, Ohio State University, University of Pittsburgh, University of Portsmouth, Princeton University, the United States Naval Observatory, and the University of Washington.

T.C.B. and Y.S.L. acknowledge partial support from grants PHY 02-16783 and PHY 08-22648; Physics Frontier Center/Joint Institute for Nuclear Astrophysics (JINA). P.R.F. acknowledges support through the Marie Curie Research Training Network ELSA (European Leadership in Space Astrometry) under contract MRTN-CT-2006-033481. P.R.F. acknowledges support through the Marie Curie Research Training Network ELSA (European Leadership in Space Astrometry) under contract MRTN-CT-2006-033481.

\section{APPENDIX}

\section{PROPERTIES OF STREAM MEMBERS}

Table 3 lists the identifications and stellar atmospheric parameters from the SSPP for stars that we have assigned to individual streams. The errors on $[\mathrm{Fe} / \mathrm{H}], T_{\text {eff }}$, and $\log g$ are internal estimates, obtained from averaging of multiple techniques. In the cases of independently observed stars, the results have been averaged. Note that in the current version of the SSPP, the astrophysical parameters have been updated to slightly different values with smaller intrinsic errors than those used in this study which are listed below.

Table 4 lists the SDSS photometry, distance estimates and errors, heliocentric radial velocities, and derived $U, V, W$ used in this study. 
Table 3

Putative Members of the Identified Streams: Astrophysical Parameters

\begin{tabular}{|c|c|c|c|c|c|c|c|c|c|c|}
\hline STREAM & IAU NAME & PLATE-MJD-FIBER & $\begin{array}{c}\alpha \\
\left({ }^{\circ}\right)\end{array}$ & $\begin{array}{c}\delta \\
\left(^{\circ}\right)\end{array}$ & {$[\mathrm{Fe} / \mathrm{H}]$} & $\sigma_{[\mathrm{Fe} / \mathrm{H}]}$ & $\begin{array}{l}T_{\text {eff }} \\
(\mathrm{K})\end{array}$ & $\begin{array}{c}\sigma_{\mathrm{T}_{\text {eff }}} \\
(\mathrm{K})\end{array}$ & $\log g$ & $\sigma_{\log g}$ \\
\hline $\mathrm{C} 1$ & SDSS J003929.56+143550.1 & $1896-53242-317$ & 9.8731813 & 14.5972557 & -1.532 & 0.032 & 5626 & 37 & 4.155 & 0.156 \\
\hline $\mathrm{C} 1$ & SDSS J004044.06+143713.5 & $1896-53242-275$ & 10.1835690 & 14.6204119 & -1.821 & 0.008 & 4618 & 89 & 4.572 & 0.294 \\
\hline $\mathrm{C} 1$ & SDSS J004209.94+004735.8 & 1905-53613-379 & 10.5414352 & 0.7932640 & -1.643 & 0.008 & 4886 & 136 & 3.570 & 0.330 \\
\hline $\mathrm{C} 1$ & SDSS J004209.94+004735.8 & $1905-53706-372$ & 10.5414352 & 0.7932640 & -1.888 & 0.078 & 4749 & 43 & 3.935 & 0.363 \\
\hline $\mathrm{C} 1$ & SDSS J011202.72+244140.1 & 2040-53384-296 & 18.0113449 & 24.6944771 & -1.457 & 0.054 & 5585 & 32 & 3.965 & 0.180 \\
\hline $\mathrm{C} 1$ & SDSS J012737.07+385706.2 & $2042-53378-555$ & 21.9044685 & 38.9517097 & -1.209 & 0.082 & 5479 & 75 & 4.489 & 0.069 \\
\hline $\mathrm{C} 1$ & SDSS J012737.07+385706.2 & 043-53351-114 & 21.9044685 & 38.9517097 & -1.345 & 0.071 & 5471 & 69 & 4.501 & 0.083 \\
\hline $\mathrm{C} 1$ & SDSS J012823.46+375742.5 & 062-53381-002 & 22.0977440 & 37.9618034 & -1.417 & 0.061 & 5121 & 50 & 4.431 & 0.151 \\
\hline $\mathrm{C} 1$ & SDSS J013010.48+144140.6 & $425-51884-467$ & 22.5436459 & 14.6946182 & -1.435 & 0.029 & 5966 & 53 & 4.209 & 0.076 \\
\hline $\mathrm{C} 1$ & SDSS J013010.48+144140.6 & $425-51898-463$ & 22.5436459 & 14.6946182 & -1.572 & 0.034 & 5978 & 48 & 4.241 & 0.092 \\
\hline $\mathrm{C} 1$ & SDSS J013249.36-091356.1 & & 23.2056828 & -9.2322512 & & & 5727 & 43 & 4.571 & 0.031 \\
\hline $\mathrm{C} 1$ & SDSS J013249.36-091356.1 & $0662-52178-426$ & 23.2056828 & -9.2322512 & -1.207 & 0.029 & 5610 & 50 & 4.425 & 0.075 \\
\hline $\mathrm{C} 1$ & SDSS J014657.63-002829.4 & $907-53265-101$ & 26.7401257 & -0.4748370 & -1.128 & 0.075 & 4841 & 92 & 4.310 & 0.215 \\
\hline $\mathrm{C} 1$ & SDSS J014657.63-002829.4 & & 26.7401257 & -0.4748370 & -1.417 & & 4744 & 16 & 4.539 & 0.068 \\
\hline $\mathrm{C} 1$ & SDSS J021054.90+224058.0 & $2046-53327-559$ & 32.7287369 & 22.6827908 & -1.465 & 0.055 & 5111 & 81 & 4.361 & 0.115 \\
\hline $\mathrm{C} 1$ & SDSS J023454.42+285631.8 & & 38.7267342 & & & & & 29 & & 0.125 \\
\hline $\mathrm{C} 1$ & SDSS J023736.38+261237.9 & $2399-53764-626$ & 39.4015694 & 26.2105236 & -1.513 & & & 63 & 4.392 & 0.053 \\
\hline $\mathrm{C} 1$ & SDSS J024458.22-003204.3 & $1664-52965-230$ & 41.2425804 & -0.5345300 & -1.523 & 0.111 & 5475 & 48 & 3.884 & 0.286 \\
\hline $\mathrm{C} 1$ & SDSS J0244 & 230 & 41.2425804 & -0.534 & -1.774 & 059 & 5465 & 41 & 3.809 & 0.270 \\
\hline $\mathrm{C} 1$ & SDSS J0257 & & 44.46 & 5.618 & -1.851 & & 5235 & 118 & 3.474 & 0.348 \\
\hline $\mathrm{C} 1$ & SDSS J0257 & & 44.46 & & -1.7 & & 5177 & 86 & 4.401 & 0.120 \\
\hline $\mathrm{C} 1$ & SDSS J032649 & & 51.70 & & & & & 53 & 4.149 & 0.072 \\
\hline $\mathrm{C} 1$ & SDSS J032649.09+054858.9 & & 51.704 & & & & & 59 & 3.708 & 0.267 \\
\hline $\mathrm{C} 1$ & SDSS J060816.47+652237.7 & 711-417 & 92.068 & & & & 5442 & 22 & 4.213 & 0.135 \\
\hline $\mathrm{C} 1$ & $51.60+010153.2$ & & 124.715 & 1.0314490 & -0.992 & & & 105 & 4.358 & 0.146 \\
\hline $\mathrm{C} 1$ & SDSS J084558.30+542552.5 & $2316-53757-628$ & 131.4929047 & 54.4312553 & -1.616 & 0.0 & 5956 & 28 & 4.330 & 0.070 \\
\hline $\mathrm{C} 1$ & SDSS J085520.23+105431.9 & $2671-54141-594$ & 133.8342743 & 10.9088564 & -1.569 & 0.085 & 5189 & 50 & 4.349 & 0.107 \\
\hline $\mathrm{C} 1$ & SDSS J094333.81+614255.3 & & 145.890 & 61.7153 & -1.6 & & & 52 & 4.591 & 0.037 \\
\hline $\mathrm{C} 1$ & SDSS J102023.87+484211.4 & $4-497$ & 155.099 & 48.703 & -1.260 & 0.031 & 580 & 39 & 4.101 & 0.106 \\
\hline $\mathrm{C} 1$ & SDSS J102023.87+484211.4 & & 155.099 & 1708 & -1.218 & & 578 & 23 & 4.081 & 0.134 \\
\hline $\mathrm{C} 1$ & SDS & & & & -1 . & & 47 & 78 & 4.408 & 0.115 \\
\hline $\mathrm{C} 1$ & SDS & & & 91 & -1 & & 58 & 24 & 4.206 & 0.118 \\
\hline $\mathrm{C} 1$ & SDSS J1246 & & & & & & 5772 & 40 & 4.234 & 0.107 \\
\hline $\mathrm{C} 1$ & SDSS J124656.06+305644.2 & & & & & & 5672 & 39 & 3.947 & 0.235 \\
\hline $\mathrm{C} 1$ & SDSS J144844.46+254959.5 & & & & & & & 27 & 3.948 & 0.157 \\
\hline $\mathrm{C} 1$ & SDSS J150940.61+005724.1 & $5-409$ & 227.4191895 & 0.9567060 & -1.183 & & 5881 & 3 & 4.448 & 0.109 \\
\hline $\mathrm{C} 1$ & SDSS J160819.86+522800.9 & $2176-54243-500$ & 242.0827789 & 52.4669037 & -1.217 & 0.057 & 5532 & 42 & 4.218 & 0.156 \\
\hline $\mathrm{C} 1$ & SDSS J162753.45+480813.1 & $0625-52145-132$ & 246.9727173 & 48.1369705 & -1.973 & & 5987 & 28 & 4.282 & 0.070 \\
\hline $\mathrm{C} 1$ & SDSS J164228.88+371457.9 & $2174-53521-623$ & 250.6203156 & 37.2494125 & -1.626 & 0.035 & 6002 & 58 & 3.569 & 0.163 \\
\hline $\mathrm{C} 1$ & SDSS J164800.02+224725.9 & & & & -1.948 & & 6050 & 29 & 4.070 & 0.068 \\
\hline $\mathrm{C} 1$ & S J174211.76+245806.5 & & 265.549 & 24.96847 & -1.526 & & 55 & 57 & 4.230 & 0.105 \\
\hline $\mathrm{C} 1$ & SDSS J233936.27+001812.8 & 11-391 & 354.901 & 0.3035480 & -1.552 & 0.071 & 5263 & 46 & 4.558 & 0.053 \\
\hline $\mathrm{C} 1$ & & & & 9000 & & & 51 & 61 & 3.592 & 0.447 \\
\hline $\mathrm{C} 1$ & $1+371115.7$ & $1880-53262-469$ & 357.639 & 37.187 & -1.209 & 0.014 & 5856 & 19 & 4.189 & 0.084 \\
\hline $\mathrm{C} 2$ & SDSS J003315.18+083239.6 & $2312-53709-367$ & 8.3132315 & 8.5443439 & -1.530 & 0.069 & 5794 & 61 & 4.237 & 0.103 \\
\hline $\mathrm{C} 2$ & SDSS J003335.38+064204.8 & $2327-53710-260$ & 8.3974247 & 6.7013278 & -1.218 & 0.044 & 5902 & 26 & 4.333 & 0.098 \\
\hline $\mathrm{C} 2$ & SDSS J003951.37+241507.1 & $2038-53327-228$ & 9.9640341 & 24.2519588 & -1.960 & 0.021 & 5921 & 35 & 4.067 & 0.066 \\
\hline $\mathrm{C} 2$ & SDSS J014003.43+224737.3 & 2044-53327-087 & & 22.7936897 & -1.808 & & 5811 & 38 & 3.907 & 0.232 \\
\hline $\mathrm{C} 2$ & SDSS J024606.36-011303.6 & $1664-52965-211$ & 41.5265083 & -1.2176800 & -1.587 & 0.059 & 4992 & 101 & 4.396 & 0.128 \\
\hline $\mathrm{C} 2$ & SDSS J024606.36-011303.6 & $1664-52973-203$ & 41.5265083 & -1.2176800 & -1.428 & 0.120 & 5027 & 75 & 4.399 & 0.125 \\
\hline $\mathrm{C} 2$ & SDSS J024606.36-011303.6 & $1511-52946-207$ & 41.5265121 & -1.2176770 & -1.753 & 0.089 & 4958 & 102 & 4.291 & 0.215 \\
\hline $\mathrm{C} 2$ & & & & & & & 56 & 35 & 3.503 & 0.262 \\
\hline $\mathrm{C} 2$ & SDSS J072805.35+363536.8 & & 112.0222778 & & & & 6076 & 41 & 3.742 & 0.142 \\
\hline $\mathrm{C} 2$ & SDSS J072957.64+404615.9 & $1734-53034-425$ & 112.4901810 & 40.7710724 & -1.362 & 0.042 & 5683 & 28 & 4.378 & 0.081 \\
\hline $\mathrm{C} 2$ & SDSS J073626.44+215322.5 & $2078-53378-352$ & 114.1101532 & 21.8895817 & -1.333 & 0.101 & 5242 & 62 & 4.517 & 0.056 \\
\hline $\mathrm{C} 2$ & SDSS J074829.13+234423.8 & $0927-52577-590$ & 117.1213913 & 23.7399406 & -1.296 & 0.053 & 5465 & 40 & 4.192 & 0.205 \\
\hline $\mathrm{C} 2$ & SDSS J075411.14+344532.4 & $0756-52577-538$ & 118.5464325 & 34.7590065 & -2.245 & 0.067 & 5907 & 43 & 4.233 & 0.124 \\
\hline $\mathrm{C} 2$ & SDSS J083939.81+064948.7 & $1298-52964-283$ & 129.9158630 & 6.8301821 & -2.080 & 0.013 & 6033 & 37 & 4.080 & 0.037 \\
\hline $\mathrm{C} 2$ & SDSS J084737.08+070118.2 & $2317-54152-362$ & 131.9044952 & 7.0217328 & -1.267 & 0.088 & 5003 & 93 & 4.546 & 0.040 \\
\hline $\mathrm{C} 2$ & DSS J090533.11+432639.5 & 0831-52294-447 & 136.3879395 & 43.4443016 & -1.729 & 0.049 & 5938 & 28 & 4.319 & 0.081 \\
\hline $\mathrm{C} 2$ & SDSS J091720.56+020254.3 & $0473-51929-423$ & 139.3356781 & 2.0484250 & -1.605 & 0.009 & 5971 & 20 & 4.300 & 0.127 \\
\hline $\mathrm{C} 2$ & SDSS J092013.59+284917.4 & $1937-53388-574$ & 140.0566254 & 28.8214874 & -2.243 & 0.025 & 6119 & 53 & 4.083 & 0.066 \\
\hline $\mathrm{C} 2$ & SDSS J092213.39+225504.1 & $2304-53762-573$ & 140.5557861 & 22.9178085 & -1.191 & 0.087 & 4964 & 85 & 4.553 & 0.050 \\
\hline $\mathrm{C} 2$ & SDSS J093632.83+473706.4 & 0834-52316-487 & 144.1368103 & 47.6184464 & -1.724 & 0.024 & 5859 & 42 & 3.894 & 0.056 \\
\hline
\end{tabular}


Table 3

(Continued)

\begin{tabular}{|c|c|c|c|c|c|c|c|c|c|c|}
\hline STREAM & IAU NAME & PLATE-MJD-FIBER & $\begin{array}{c}\alpha \\
\left(^{\circ}\right) \\
\end{array}$ & $\begin{array}{c}\delta \\
\left({ }^{\circ}\right) \\
\end{array}$ & {$[\mathrm{Fe} / \mathrm{H}]$} & $\sigma_{[\mathrm{Fe} / \mathrm{H}]}$ & $\begin{array}{l}T_{\text {eff }} \\
(K)\end{array}$ & $\begin{array}{c}\sigma_{\mathrm{T}_{\text {eff }}} \\
(\mathrm{K})\end{array}$ & $\log g$ & $\sigma_{\log g}$ \\
\hline $\mathrm{C} 2$ & SDSS J094018.33-003356.5 & 0476-52314-010 & 145.0763855 & -0.5657040 & -1.917 & 0.039 & 5883 & 52 & 4.319 & 0.092 \\
\hline $\mathrm{C} 2$ & SDSS J095506.81+114300.7 & & 148.7783813 & 11.7168503 & -1.357 & 0.050 & 5959 & 38 & 4.349 & 0.114 \\
\hline $\mathrm{C} 2$ & SDSS J095908.86+005645.0 & 0500-51994-087 & 149.7869110 & 0.9458360 & -1.706 & 0.083 & 5584 & 77 & 4.303 & .102 \\
\hline $\mathrm{C} 2$ & SDSS J101455.05+253710.0 & 2386-54064-034 & 153.7293854 & 25.6194496 & -0.989 & 0.114 & 5263 & 92 & 4.419 & 0.084 \\
\hline $\mathrm{C} 2$ & SDSS J104621.93+004321.8 & $2559-54208-362$ & 161.5913849 & 0.7227150 & -1.512 & 0.053 & 5425 & 54 & 4.594 & 0.043 \\
\hline $\mathrm{C} 2$ & SDSS J105039.60-004524.8 & $2559-54208-050$ & 162.6649933 & -0.7568800 & -1.520 & 0.106 & 4812 & 31 & 4.492 & .073 \\
\hline $\mathrm{C} 2$ & SDSS J105338.83+481716.5 & 2390-54094-469 & 163.4117889 & 48.2879257 & & 0.062 & 5077 & 65 & 3.734 & 0.422 \\
\hline $\mathrm{C} 2$ & SDSS J105338.83+481716.5 & $2410-54087-474$ & 163.4117889 & 48.2879257 & -2.261 & 0.038 & 5086 & 70 & 3.614 & 0.344 \\
\hline $\mathrm{C} 2$ & SDSS J105649.28+014446.2 & 0507-52353-029 & 164.2053528 & 1.7461530 & -1.713 & 0.049 & 5637 & 35 & 3.709 & 0.303 \\
\hline $\mathrm{C} 2$ & SDSS J111101.99+103838.3 & $2393-54156-322$ & 167.7583160 & 10.6439629 & -1.216 & 0.077 & 5145 & 49 & 4.396 & 0.092 \\
\hline $\mathrm{C} 2$ & SDSS J113811.21+482056.1 & $1444-53054-378$ & 174.5467072 & 48.3489304 & -1.244 & 0.027 & 5748 & 47 & 3.986 & 0.158 \\
\hline $\mathrm{C} 2$ & SDSS J115028.60+015703.2 & & 177.6191711 & & & & & 43 & & 0.102 \\
\hline $\mathrm{C} 2$ & SDSS J122249.57+010247.7 & & & & & & & 68 & & \\
\hline $\mathrm{C} 2$ & SDSS J124601.97+285517.0 & $2239-53726-293$ & 191.5082245 & 28.9213829 & -1.606 & & & 36 & 4.215 & .072 \\
\hline $\mathrm{C} 2$ & SDSS J124601.97+285517.0 & & 191.5082245 & 28.9213829 & -1.738 & & 6002 & 35 & & .093 \\
\hline $\mathrm{C} 2$ & SDSS J15293 & & 32.412 & 735 & -2.367 & & & 91 & & .230 \\
\hline $\mathrm{C} 2$ & SDSS J16114 & & 242.933 & 53.73 & & & 4690 & 82 & 4.609 & .301 \\
\hline $\mathrm{C} 2$ & SDSS J161513.53+070012.5 & & & & & & & 44 & 3.922 & 0.103 \\
\hline $\mathrm{C} 2$ & SDSS J161513.53+070012.5 & & 243.8063660 & & & & 5860 & 60 & 4.030 & 0.147 \\
\hline $\mathrm{C} 2$ & SDSS J163043.32+633133.6 & & 247.6804962 & 63.5260124 & & & & 95 & 4.495 & 0.085 \\
\hline $\mathrm{C} 2$ & SDSS J172425.97+265121.1 & 2193-53888-182 & 261.1082153 & 26.8558598 & & & 4437 & 72 & 4.700 & 0.157 \\
\hline $\mathrm{C} 2$ & SDSS J220031.01+001657.2 & $106-52912-506$ & 330.1292114 & 0.2825420 & -1.313 & & & 64 & 4.536 & 0.109 \\
\hline $\mathrm{C} 2$ & SDSS J223948.41+232244.7 & $252-53565-364$ & 339.9516907 & 23.3790913 & -1.742 & & & 87 & 4.050 & 0.317 \\
\hline $\mathrm{C} 2$ & SDSS J223948.41+232244.7 & $252-53613-382$ & 339.9516907 & 23.3790913 & -1 & & & 79 & & 0.381 \\
\hline $\mathrm{C} 2$ & SDSS J224213.28+222015.7 & 25253565.278 & 340.5553 & 22.3376 & & & & 98 & & 0.237 \\
\hline $\mathrm{C} 2$ & SDSS J224213.28+222015.7 & $2252-53613-251$ & 340.555 & 22.337 & -1 & & & 54 & 3.172 & 0.209 \\
\hline $\mathrm{C} 2$ & SDSS J224514.28-003350.8 & & 341.309 & 130 & & & & 40 & 3.680 & .381 \\
\hline $\mathrm{C} 2$ & SDS & & & & & & & 64 & & .325 \\
\hline $\mathrm{C} 2$ & SDSS & & & & & & 56 & 61 & 3.569 & .073 \\
\hline $\mathrm{C} 2$ & $9.91+000557.3$ & & & & & & & 44 & 3.499 & 0.285 \\
\hline $\mathrm{C} 2$ & SDSS J233835.27+075729.9 & & & & & & & 30 & 4.418 & 0.098 \\
\hline $\mathrm{C} 2$ & SDSS J234345.10+002735.9 & & 355.9379272 & & & & 46 & 103 & 4.631 & 0.217 \\
\hline $\mathrm{C} 3$ & SDSS J003149.73-004244.2 & & 7.9571881 & 2680 & -1 & & & 94 & & 0.110 \\
\hline $\mathrm{C} 3$ & SDSS J003542.54+061441.9 & $2312-53709-123$ & 8.9272423 & 6.2449832 & -1.286 & 0.064 & 5517 & 47 & 4.363 & 0.092 \\
\hline $\mathrm{C} 3$ & SDSS J003609.72-004756.4 & 1135-53024-095 & 9.0404949 & -0.7989910 & -2.043 & 0.065 & & 63 & 4.332 & 0.140 \\
\hline $\mathrm{C} 3$ & SDSS J003947.76+003919.3 & $1904-53682-340$ & & 0.6553600 & -1.758 & & 5626 & 49 & 3.953 & 0.242 \\
\hline $\mathrm{C} 3$ & SDSS J003947.76+003919.3 & & 9.9490156 & 0.6553640 & -1.9 & & & 80 & 3.974 & 0.277 \\
\hline $\mathrm{C} 3$ & 919.3 & & & & & & & 54 & 4.052 & 0.176 \\
\hline $\mathrm{C} 3$ & 2.0 & & 11.6 & & & & 60 & 47 & 3.472 & 0.053 \\
\hline $\mathrm{C} 3$ & 8.9 & & & & & & & 38 & 14 & 0.074 \\
\hline $\mathrm{C} 3$ & $21022.12+214531.8$ & -097 & 1631 & 329 & -1.638 & & 5356 & 31 & 4.030 & 0.149 \\
\hline $\mathrm{C} 3$ & & & & -7.9979420 & & & & 48 & 3.796 & 0.257 \\
\hline $\mathrm{C} 3$ & SDSS J032120.59+060355.3 & & 50.3357887 & 6.0653648 & -2.004 & & 5376 & 34 & 3.724 & 0.271 \\
\hline $\mathrm{C} 3$ & SDSS J032120.59+060355.3 & $334-53730-339$ & 50.3357887 & 6.0653648 & -1.987 & 0.036 & 5318 & 54 & 3.510 & 0.274 \\
\hline $\mathrm{C} 3$ & SDSS J081525.99+362755.8 & & 123.8583069 & 36.4654922 & -1.886 & & 5744 & 63 & 4.269 & 0.056 \\
\hline $\mathrm{C} 3$ & SDSS J082533.57+182230.9 & $271-53726-612$ & 126.3898697 & 18.3752556 & -2.527 & 0.026 & 6229 & 49 & 3.550 & 0.294 \\
\hline $\mathrm{C} 3$ & SDSS J082533.57+182230.9 & $273-53709-479$ & 126.3898697 & 18.3752556 & -2.213 & 0.023 & 6259 & 41 & 3.683 & 0.270 \\
\hline $\mathrm{C} 3$ & SDSS J084043.12+095140.2 & $573-54061-093$ & 130.1796570 & 9.8611660 & -1.895 & 0.007 & 5886 & 40 & 4.445 & 0.108 \\
\hline $\mathrm{C} 3$ & SDSS J092032.03+231836.5 & & & & & & & 35 & 87 & 0.112 \\
\hline $\mathrm{C} 3$ & S J092032.03+231836.5 & $14-124$ & 140.13 & 3.3101311 & -1.5 & 0.009 & 611 & 47 & 4.209 & 0.124 \\
\hline $\mathrm{C} 3$ & $2824.65+064514.5$ & $2382-54169-103$ & 142.1027069 & 6.7540302 & -1.765 & 0.065 & 4894 & 75 & 3.847 & 0.316 \\
\hline $\mathrm{C} 3$ & SDSS J094030.57+381706.9 & $1276-53035-430$ & 145.1273651 & 38.2852554 & -2.276 & 0.029 & 5954 & 28 & 3.983 & 0.082 \\
\hline $\mathrm{C} 3$ & SDSS J094934.58+001711.5 & 0267-51608-359 & 147.3940735 & 0.2865320 & -1.555 & 0.028 & 6165 & 63 & 3.380 & 0.140 \\
\hline $\mathrm{C} 3$ & SDSS J100406.88+060336.0 & 0996-52641-311 & 151.0286560 & 6.0600109 & -2.005 & 0.026 & 5815 & 58 & 4.019 & 0.134 \\
\hline $\mathrm{C} 3$ & SDSS J100908.52+013225.0 & $0502-51957-231$ & 152.2854919 & 1.5402700 & -2.224 & 0.024 & 6035 & 42 & 4.212 & 0.064 \\
\hline $\mathrm{C} 3$ & SDSS J101140.34+354038.8 & $2387-53770-520$ & 152.9180756 & 35.6774483 & -2.218 & 0.107 & 4801 & 83 & 3.693 & 0.340 \\
\hline $\mathrm{C} 3$ & SDSS J101749.41+404601.6 & $1357-53034-557$ & 154.4558868 & 40.7671013 & -2.233 & 0.038 & 5410 & 61 & 3.885 & 0.208 \\
\hline $\mathrm{C} 3$ & SDSS J103459.70+082335.9 & $1240-52734-254$ & 158.7487640 & 8.3933039 & -1.787 & 0.039 & 6014 & 31 & 4.256 & 0.111 \\
\hline $\mathrm{C} 3$ & SDSS J103658.20+163609.3 & $2594-54177-392$ & 159.2425232 & 16.6025944 & -2.659 & 0.036 & 5658 & 154 & 3.029 & 0.441 \\
\hline $\mathrm{C} 3$ & SDSS J104827.81+005524.0 & 2389-54213-444 & & & & & & 65 & 3.442 & 0.115 \\
\hline $\mathrm{C} 3$ & SDSS J104838.61+005839.4 & $2389-54213-443$ & 162.1608887 & 0.9776190 & & 0.022 & 6063 & 36 & 4.351 & 0.140 \\
\hline $\mathrm{C} 3$ & SDSS J104838.61+005839.4 & $2409-54210-508$ & 162.1608887 & 0.9776190 & -1.930 & 0.049 & 6071 & 64 & 4.274 & 0.118 \\
\hline $\mathrm{C} 3$ & SDSS J104838.61+005839.4 & $2559-54208-443$ & 162.1608887 & 0.9776190 & -1.827 & 0.031 & 6038 & 53 & 4.098 & 0.044 \\
\hline
\end{tabular}


Table 3

(Continued)

\begin{tabular}{|c|c|c|c|c|c|c|c|c|c|c|}
\hline STREAM & IAU NAME & PLATE-MJD-FIBER & $\begin{array}{c}\alpha \\
\left(^{\circ}\right) \\
\end{array}$ & $\begin{array}{c}\delta \\
\left(^{\circ}\right)\end{array}$ & {$[\mathrm{Fe} / \mathrm{H}]$} & $\sigma_{[\mathrm{Fe} / \mathrm{H}]}$ & $\begin{array}{l}T_{\text {eff }} \\
(\mathrm{K})\end{array}$ & $\begin{array}{c}\sigma_{\mathrm{T}_{\text {eff }}} \\
(\mathrm{K})\end{array}$ & $\log g$ & $\sigma_{\log g}$ \\
\hline $\mathrm{C} 3$ & SDSS J104838.61+005839.4 & $2569-54234-442$ & 162.1608887 & 0.9776190 & -1.914 & 0.043 & 6099 & 50 & 4.290 & 0.121 \\
\hline $\mathrm{C} 3$ & SDSS J105236.88-004816.8 & 2389-54213-003 & 163.1536865 & -0.8046550 & -0.805 & & 5343 & 109 & 4.614 & \\
\hline $\mathrm{C} 3$ & SDSS J105236.88-004816.8 & 559-54208-002 & & -0.8046550 & & & & 26 & 4.395 & 0.136 \\
\hline $\mathrm{C} 3$ & SDSS J105643.51+480559.6 & $2410-54087-160$ & & 48.0998764 & & & & 104 & 4.340 & \\
\hline $\mathrm{C} 3$ & SDSS J124923.70+291857.5 & $2457-54180-103$ & 192.3487396 & 29.3159676 & -1.395 & 0.032 & 5830 & 42 & 4.329 & 0.083 \\
\hline $\mathrm{C} 3$ & SDSS J160840.68+524221.9 & $2176-54243-487$ & 242.1694946 & 52.7060776 & -1.484 & 0.025 & 5554 & 30 & 4.266 & 0.137 \\
\hline C3 & SDSS J161247.16+194440.3 & 2205-53793-018 & 243.1965179 & 19.7445164 & -2.100 & 0.012 & 5990 & 41 & 4.079 & 0.142 \\
\hline $\mathrm{C} 3$ & DSS J161247.16+194440.3 & $2206-53795-2$ & 243.1965179 & 19.7445164 & -1.982 & & 6062 & 33 & 4.326 & 0.141 \\
\hline C3 & SDSS J171058.39+430326.4 & $2256-53613-338$ & 257.7432861 & 43.0573235 & -1.501 & 0.192 & 4497 & 101 & 4.352 & 0.095 \\
\hline C3 & SDSS J172433.72+260332.6 & 2182-53905-214 & 261.1405029 & 26.0590591 & -2.267 & 0.026 & 4943 & 151 & 3.319 & 0.482 \\
\hline $\mathrm{C} 3$ & SDSS J204435.17-005117.0 & $1908-53239-135$ & 311.1465454 & -0.8547190 & -1.808 & 0.028 & 5453 & 46 & 3.236 & 0.179 \\
\hline $\mathrm{C} 3$ & SDSS J233626.78+100057.5 & $2622-54095-361$ & 354.1115723 & 10.0159616 & -1.274 & & 5416 & 64 & 3.087 & 0.252 \\
\hline $\mathrm{C} 3$ & SDSS J233912.75-004742.7 & & & -0.7952000 & & & & 34 & 4.075 & \\
\hline $\mathrm{C} 3$ & & & & & & & & 36 & & \\
\hline $\mathrm{C} 3$ & & & & & & & & 36 & 4.102 & \\
\hline $\mathrm{C} 4$ & SDSS J011441.01+010914.8 & -414 & 18.670 & 1.1541 & -2.389 & 0.011 & & 45 & 3.691 & 0.114 \\
\hline $\mathrm{C} 4$ & SDSS J023158.11+255029.1 & & 37.992 & 25.8414 & -2.267 & & 5907 & 48 & 4.052 & 0.255 \\
\hline $\mathrm{C} 4$ & 5.9 & & 38.64 & -8.798 & -2 & & 6030 & 40 & 3.326 & 0.179 \\
\hline $\mathrm{C} 4$ & SDSS J024 & & & -0.16 & & 0.030 & 5820 & 135 & 4.280 & 0.215 \\
\hline $\mathrm{C} 4$ & SDSS J024418.33-000947.9 & & & & & & & 34 & 4.376 & \\
\hline $\mathrm{C} 4$ & SDSS J081918.72+390006.5 & & 124.8280106 & 39.0018082 & & & & 34 & 4.516 & 0.070 \\
\hline $\mathrm{C} 4$ & PSS J103649.93+121219.8 & $1600-53090-378$ & 159.2080383 & 12.2055120 & -3.037 & & 5938 & 77 & 3.355 & 0.324 \\
\hline $\mathrm{C} 4$ & DSS J105230.56+314419.2 & -213 & 3.1273499 & 31.7386761 & -2 & & & 51 & 3.852 & 0.121 \\
\hline $\mathrm{C} 4$ & DSS J105424.31+372221.2 & $4-120$ & 63.6012878 & 37.3725 & -2.153 & & 54 & 72 & 3.848 & 0.298 \\
\hline $\mathrm{C} 4$ & DSS J113626.47+020433.3 & $0513-5$ & 7410 & 2.0759 & -2 & & & 78 & 4.116 & \\
\hline $\mathrm{C} 4$ & DSS J124342.45+101708.0 & & & 10.2855 & & & & 46 & 4.232 & \\
\hline $\mathrm{C} 4$ & SDSS J124615.79-090738.0 & & & -9.1272 & -2 & & & 11 & 3.497 & 0.310 \\
\hline $\mathrm{C} 4$ & & & & & & & & 60 & 3.882 & 0.108 \\
\hline $\mathrm{C} 4$ & & & & & & & 56 & 40 & 3.209 & 0.223 \\
\hline $\mathrm{C} 4$ & & & & & -2 . & & 60 & 54 & 3.562 & 0.114 \\
\hline $\mathrm{C} 4$ & $14+135708.8$ & & & & -2 . & 0.0 & 59 & 68 & 3.869 & 0.235 \\
\hline $\mathrm{C} 4$ & DSS J163713.47+632122.9 & & & & & & & 107 & 4.428 & 0.073 \\
\hline $\mathrm{C} 4$ & DSS J174259.25+244823.2 & & & & -2 & & & 40 & 3.009 & 0.300 \\
\hline $\mathrm{C} 4$ & DSS J200336.15+600006.9 & -279 & 300.9006348 & 60.0019035 & -0.441 & 0.09 & 49 & 88 & 3.472 & 0.121 \\
\hline $\mathrm{C} 4$ & SDSS J211011.44+002324.9 & $1918-53240-522$ & 317.5476685 & 0.3902380 & -2.143 & 0.037 & & 41 & 3.077 & 0.275 \\
\hline $\mathrm{C} 4$ & SDSS J215433.88-071000.4 & 0716-52203-323 & 328.6411438 & -7.1667900 & -2.222 & 0.013 & 6025 & 26 & 4.328 & 0.095 \\
\hline $\mathrm{C} 4$ & SDSS J234017.29-003337.6 & & 355.0720215 & -0.5604550 & -1.111 & & & 61 & 4.511 & 0.068 \\
\hline $\mathrm{C} 4$ & & & & & & & & 6 & 4.253 & \\
\hline $\mathrm{C} 4$ & & & & & & & & 12 & 4.235 & 0.118 \\
\hline $\mathrm{C} 4$ & & & & & -0 . & & & 23 & 4.204 & 0.116 \\
\hline H99 & & & & & -2 & & 58 & 49 & 4.353 & 0.070 \\
\hline H99 & 812.0 & -563 & 3792 & 9940 & -1.273 & 0.0 & 5602 & 34 & 4.184 & 0.175 \\
\hline H99 & DSS J004755.30+143355.6 & $242-109$ & 11.9804010 & 14.5654478 & -1.659 & 0.049 & 5194 & 54 & 4.076 & 0.235 \\
\hline H99 & DSS J012316.16-001508.5 & $2209-172$ & 20.8173447 & -0.2523500 & -2.168 & 0.057 & 5423 & 59 & 3.786 & 0.301 \\
\hline H99 & SS J014552.75+134648.5 & & 26.4698048 & 13.7801332 & -2.168 & & 5328 & 73 & 3.801 & 0.229 \\
\hline H99 & DSS J014858.66+001851.5 & $1906-53293-633$ & 7.2444191 & 0.3143000 & -1.913 & & 4952 & 70 & 4.373 & 0.046 \\
\hline H99 & DSS J015218.06-003227.2 & $1076-52914-085$ & 8.0752354 & -0.5408920 & -1.032 & & 5151 & 30 & 4.420 & 0.130 \\
\hline H99 & DSS J021139.03+060824.3 & 306-53726-188 & 2.9126167 & 6.1400919 & -1.736 & 0.061 & 5464 & 52 & 4.049 & 0.205 \\
\hline H99 & & & & 1.2292100 & -1.711 & & 53 & 55 & 3.829 & 0.341 \\
\hline H99 & & & & & & & & & 3.741 & \\
\hline H99 & & & & & & & & 44 & 3.729 & 0.358 \\
\hline H99 & $13+0113452$ & & & 1.2292100 & -1.838 & 0 & 533 & 48 & 3.819 & 0.369 \\
\hline H99 & SDSS J022207.13+011345.2 & 0406-52238-367 & 35.5297279 & 1.2292100 & -1.761 & 0.022 & 5357 & 60 & 3.805 & 0.353 \\
\hline H99 & SDSS J022207.13+011345.2 & $1558-53271-361$ & 35.5297279 & 1.2292100 & -1.729 & 0.028 & 5351 & 51 & 3.684 & 0.366 \\
\hline H99 & SDSS J024809.01-005121.7 & $1664-52965-135$ & 42.0375404 & -0.8560400 & -1.726 & 0.031 & 5813 & 49 & 4.320 & 0.068 \\
\hline H99 & DSS J024809.01-005121.7 & $1664-52973-137$ & 42.0375404 & -0.8560400 & -1.723 & 0.042 & 5846 & 44 & 4.308 & 0.071 \\
\hline H99 & DSS J081654.87+265422.0 & $1266-52709-395$ & 124.2286072 & 26.9061050 & -1.999 & 0.037 & 6038 & 25 & 4.279 & 0.094 \\
\hline H99 & SSS J092940.69+410552.2 & $939-52636-160$ & 142.4195404 & 41.0978432 & -2.217 & 0.032 & 5977 & 67 & 3.877 & 0.088 \\
\hline H99 & DSS J101829.06+650218.9 & 0488-51914-532 & 154.6210632 & 65.0385818 & -1.870 & 0.028 & 5907 & 31 & 3.866 & 0.152 \\
\hline & & & & & & & & 74 & 4.193 & 0.103 \\
\hline H99 & & 0279-51608-349 & 167.8815155 & 0.0599980 & & & & 50 & 4.229 & 0.115 \\
\hline H99 & & & & & & & 5653 & 55 & 4.191 & 0.126 \\
\hline H99 & SDSS J125635.48+403725.3 & 2021-53475-369 & 194.1478271 & 40.6236916 & & 0.032 & 5913 & 51 & 4.225 & 0.078 \\
\hline H99 & SDSS J163516.45+143604.5 & $2209-53907-540$ & 248.8185272 & 14.6012478 & -2.297 & 0.011 & 5744 & 34 & 3.771 & 0.242 \\
\hline
\end{tabular}


Table 3

(Continued)

\begin{tabular}{|c|c|c|c|c|c|c|c|c|c|c|}
\hline STREAM & IAU NAME & PLATE-MJD-FIBER & $\begin{array}{c}\alpha \\
\left(^{\circ}\right) \\
\end{array}$ & $\begin{array}{c}\delta \\
\left(^{\circ}\right)\end{array}$ & {$[\mathrm{Fe} / \mathrm{H}]$} & $\sigma_{[\mathrm{Fe} / \mathrm{H}]}$ & $\begin{array}{l}T_{\text {eff }} \\
(\mathrm{K})\end{array}$ & $\begin{array}{c}\sigma_{\mathrm{T}_{\text {eff }}} \\
(\mathrm{K})\end{array}$ & $\log g$ & $\sigma_{\log g}$ \\
\hline H99 & SDSS J172312.11+275319.5 & 2182-53905-409 & 260.8004761 & 27.8887577 & -1.855 & 0.049 & 5307 & 79 & 3.806 & 0.222 \\
\hline H99 & DSS J211224.90+002453.8 & $1918-53240-565$ & 318.1037598 & 0.4149530 & -2.154 & 0.026 & 6054 & 31 & 4.122 & 0.146 \\
\hline H99 & SDSS J211224.91+002453.8 & 0985-52431-625 & 318.1037903 & 0.4149580 & -2.124 & 0.017 & 6083 & 40 & 3.934 & 0.097 \\
\hline H99 & SDSS J211224.91+002453.8 & 986-52443-418 & 318.1037903 & 0.4149580 & -2.249 & 0.027 & 6060 & 30 & 4.214 & 0.067 \\
\hline H99 & SDSS J211224.91+002453.8 & $1025-53239-622$ & 318.1037903 & 0.4149580 & -2.364 & 0.023 & 6016 & 45 & 4.038 & 0.101 \\
\hline H99 & SDSS J211224.91+002453.8 & $1026-52558-387$ & 318.1037903 & 0.4149580 & -2.100 & 0.028 & 6124 & 37 & 3.977 & 0.185 \\
\hline H99 & SDSS J211224.91+002453.8 & $1112-53180-432$ & 318.1037903 & 0.4149580 & -2.156 & 0.070 & 6134 & 47 & 3.919 & 0.141 \\
\hline H99 & SDSS J211224.91+002453.8 & $1523-52937-427$ & 318.1037903 & 0.4149580 & -2.091 & 0.032 & 6118 & 22 & 3.859 & 0.188 \\
\hline H99 & SDSS J222048.55+132527.1 & $0736-52221-628$ & 335.2022705 & 13.4241829 & -1.477 & 0.020 & 5754 & 18 & 3.956 & 0.216 \\
\hline H99 & SDSS J224938.70-083003.6 & $0723-52201-568$ & 342.4112244 & -8.5010118 & -1.100 & & & 17 & 4.210 & \\
\hline H99 & & $1902-53271-5$ & 356.92703 & 0.0480870 & & 0.027 & & 35 & 4.422 & 0.087 \\
\hline RAVE & SDSS J013839.30-093045.6 & 1914-53729-1 & 24.6637535 & -9.5126638 & -1.371 & 0.068 & 5369 & 29 & 4.423 & 0.106 \\
\hline RAVE & SDSS J013839.30-093045.6 & & 35 & -9.5126 & -1.300 & 122 & & 49 & 4.126 & 0.243 \\
\hline RAVE & SDSS J013839.30-093045.6 & & 35 & -9.51 & -1 . & & & 24 & 4.114 & 0.231 \\
\hline RAVE & SDSS J014203.92-090422.8 & & 31 & -9.07 & -0 . & & & 60 & 4.657 & 0.026 \\
\hline RAVE & SDSS J01420 & & & -9.07 & & & & 72 & 4.579 & 0.036 \\
\hline RAVE & SDSS J014203.92-090422.8 & & & -9.0730104 & & & & 63 & & 0.039 \\
\hline RAVE & SDSS J014710.87-001335.3 & & & -0.2264600 & & & & 58 & 2.972 & 0.187 \\
\hline RAVE & DSS J014710.87-001335.3 & & 26.795 & -0.226 & -1.302 & 0.033 & & 28 & 3.318 & 0.147 \\
\hline RAVE & DSS J014710.87-001335.3 & & 26.79529 & -0.226 & -1.422 & & & 45 & & 0.111 \\
\hline RAVE & DSS J015905.74-003222.3 & & 29.7739 & -0.53 & & & & 41 & & 0.083 \\
\hline RAVE & DSS J015905.74-003222.3 & & 29.773 & & & & & 66 & & \\
\hline RAVE & DSS J033942.37-060709.4 & & & & -1 . & & & 22 & 4.628 & 0.069 \\
\hline RAVE & DSS J082021.79+391100.7 & 2670 & 125.09 & 39.1 & -1 . & 8 & & 40 & 4.115 & 227 \\
\hline RAVE & & & & & & & & 1 & & \\
\hline RAVE & & & & & & & & 39 & & \\
\hline RAVE & & & & & & & & 46 & & 278 \\
\hline RAVE & & & & & & & & 40 & & \\
\hline RAVE & $311.63-164457.4$ & & & 57 & & & & 38 & & 0.075 \\
\hline RAVE & SDSS J111434.17+094641.6 & & & & & & & 52 & & 0.066 \\
\hline RAVE & SS J122210.68+002445.2 & & & 520 & -1 . & & 47 & 59 & & 0.078 \\
\hline RAVE & DSS J123456.17+515326.2 & $84-52374-038$ & 8.7340240 & 51.8906097 & -1.108 & & & 77 & & 0.054 \\
\hline RAVE & DSS J132647.54+295741.2 & & 201.69810 & .9614429 & -2.036 & & & 37 & & 0.100 \\
\hline RAVE & DSS J154838.74+445401.8 & & & .9004936 & -1 . & & & 14 & & 0.103 \\
\hline RAVE & & & 3 & & -1 . & & & 43 & & 0.091 \\
\hline RAVE & & & 340.0 & & -1 . & & & 53 & & 0.176 \\
\hline RAVE & & & & & & 0.0 & 56 & 39 & 4.3 & 087 \\
\hline RAVE & & & & & & 0.0 & 54 & 30 & 3.2 & 0.145 \\
\hline S3 & $83+005338.6$ & & 32 & 0620 & -1 . & 0.047 & 5994 & 32 & 3.828 & 0.106 \\
\hline S3 & & & & & & & & 42 & & 0.082 \\
\hline S3 & DSS J002331.83+005338.6 & & & 0.8940620 & & & 6004 & 46 & & 0.095 \\
\hline S3 & DSS J002331.83+005338.6 & & 5.8826432 & 0.8940620 & -1.406 & 0.02 & 5920 & 47 & 3.697 & 0.092 \\
\hline S3 & DSS J002331.83+005338.6 & & 5.8826432 & 0.8940620 & -1.468 & 0.07 & 5952 & 36 & 3.820 & 0.091 \\
\hline S3 & DSS J002331.83+005338.6 & & 5.8826432 & & -1.436 & & 585 & 50 & 3.670 & 0.130 \\
\hline S3 & DSS J002331.83+005338.6 & & & 0.8940620 & -1.328 & 0.029 & 597 & 34 & 3.940 & 0.151 \\
\hline S3 & DSS J002331.83+005338.6 & & 5.8826432 & 0.8940620 & & 0.01 & 598 & 30 & 3.781 & 0.109 \\
\hline S3 & 20 & & & & & & & 18 & & \\
\hline S3 & & & & & & & 59 & 6 & & 0.099 \\
\hline S3 & 04.1 & & 26000 & 330 & -1.705 & 0.03 & 548 & 54 & 4.111 & 0.214 \\
\hline S3 & DSS J025220.13+335550.4 & $9-193$ & 8547 & 6755 & -1.481 & 0.043 & 5616 & 35 & 4.252 & 0.179 \\
\hline S3 & DSS J035419.29-045941.0 & $2051-53738-334$ & 58.5803757 & -4.9947348 & -1.106 & 0.049 & 5839 & 51 & 4.368 & 0.030 \\
\hline S3 & DSS J035419.29-045941.0 & $2071-53741-365$ & 58.5803757 & -4.9947348 & & 0.040 & 5884 & 49 & 4.410 & 0.102 \\
\hline S3 & DSS J063233.61+273645.5 & $2696-54167-448$ & 98.1400604 & 27.6126442 & -1.603 & 0.162 & 4742 & 88 & 4.172 & 0.164 \\
\hline S3 & DSS J071848.45+313202.6 & 2677-54180-432 & 109.7018814 & 31.5340672 & -1.018 & 0.044 & 5510 & 75 & 4.493 & 0.084 \\
\hline S3 & SS J072100.85+411425.4 & & 110.2535477 & 41.2403831 & -0.924 & 0.038 & 5907 & 24 & 4.475 & 0.090 \\
\hline S3 & SDSS J073614.44+411329.5 & $2683-54153-546$ & 114.0601578 & 41.2248535 & -1.502 & 0.062 & 565 & 55 & 4.340 & 0.096 \\
\hline S3 & SDSS J084702.66+105403.2 & & & 10.9008980 & & 0.010 & 55 & 28 & 4.283 & 0.141 \\
\hline S3 & & & & 39.5526695 & & & 57 & 55 & 4.212 & 0.050 \\
\hline S3 & SDSS J092513.14+071055.3 & & & & & & 5011 & 71 & 4.243 & 0.044 \\
\hline S3 & & & & & & 0.017 & 5264 & 48 & 4.375 & 0.092 \\
\hline S3 & SDSS J170051.55+390528.4 & 2181-53524-143 & 255.2147980 & 39.0912323 & & 0.075 & 5666 & 43 & 4.343 & 0.100 \\
\hline S3 & SDSS J180538.01+241532.1 & $2195-54234-462$ & 271.4083862 & 24.2589111 & -1.902 & 0.068 & 4761 & 50 & 3.880 & 0.332 \\
\hline S3 & SDSS J215703.65+003723.0 & $1474-52933-429$ & 329.2651978 & 0.6230650 & -2.485 & 0.107 & 4843 & 125 & 3.455 & 0.605 \\
\hline S3 & SDSS J224417.24+001749.1 & $1900-53262-487$ & 341.0718384 & 0.2969680 & -1.797 & 0.041 & 4519 & 97 & 4.609 & 0.301 \\
\hline
\end{tabular}


Table 3

(Continued)

\begin{tabular}{|c|c|c|c|c|c|c|c|c|c|c|}
\hline STREAM & IAU NAME & PLATE-MJD-FIBER & $\begin{array}{c}\alpha \\
\left(^{\circ}\right) \\
\end{array}$ & $\begin{array}{c}\delta \\
\left(^{\circ}\right)\end{array}$ & {$[\mathrm{Fe} / \mathrm{H}]$} & $\sigma_{[\mathrm{Fe} / \mathrm{H}]}$ & $\begin{array}{l}T_{\text {eff }} \\
(\mathrm{K})\end{array}$ & $\begin{array}{c}\sigma_{\mathrm{T}_{\text {eff }}} \\
(\mathrm{K})\end{array}$ & $\log g$ & $\sigma_{\log g}$ \\
\hline S3 & SDSS J224650.01+004000.4 & $1900-53262-547$ & 341.7084045 & 0.6667650 & -1.387 & 0.043 & 4753 & 48 & 4.668 & 0.068 \\
\hline S3 & SDSS J230110.77+054925.9 & $2310-53710-098$ & 345.2948608 & 5.8238521 & -2.464 & 0.055 & 5965 & 47 & 3.985 & 0.214 \\
\hline $\mathrm{S} 3 \mathrm{C} 2$ & SDSS J014430.48+010052.3 & $1906-53293-522$ & 26.1270161 & 1.0145240 & -1.662 & & 5002 & 90 & 4.377 & 0.150 \\
\hline $\mathrm{S} 3 \mathrm{C} 2$ & SDSS J021049.15+064131.3 & 2306-53726-232 & 32.7048035 & 6.6920271 & -1.633 & 0.074 & 5531 & 50 & 3.958 & 0.227 \\
\hline $\mathrm{S} 3 \mathrm{C} 2$ & SDSS J024116.14+291410.5 & $2442-54065-565$ & 40.3172493 & 29.2362576 & -1.727 & 0.092 & 4931 & 69 & 4.258 & 0.208 \\
\hline $\mathrm{S} 3 \mathrm{C} 2$ & SDSS J075917.15+191709.9 & $1922-53315-180$ & 119.8214569 & 19.2860889 & -1.652 & 0.027 & 5917 & 39 & 4.309 & 0.102 \\
\hline $\mathrm{S} 3 \mathrm{C} 2$ & SDSS J084011.10+130755.4 & $2426-53795-587$ & 130.0462646 & 13.1320667 & -1.400 & 0.056 & 5398 & 9 & 4.523 & 0.062 \\
\hline $\mathrm{S} 3 \mathrm{C} 2$ & SDSS J085412.39+054042.3 & 2332-54149-069 & 133.5516205 & 5.6784272 & -1.351 & 0.032 & 5005 & 57 & 4.378 & 0.105 \\
\hline $\mathrm{S} 3 \mathrm{C} 2$ & SDSS J111556.02+103009.7 & 2393-54156-531 & 168.9834290 & 10.5026827 & -1.688 & 0.012 & 6044 & 57 & 4.301 & 0.107 \\
\hline $\mathrm{S} 3 \mathrm{C} 2$ & SDSS J204541.61-050231.6 & $1916-53269-520$ & 311.4233704 & -5.0421152 & -1.917 & 0.038 & 5977 & 34 & 4.384 & 0.059 \\
\hline $\mathrm{S} 3 \mathrm{C} 2$ & SDSS J214325.56-001037.6 & 0990-52465-157 & 325.8565063 & -0.1771210 & -2.201 & 0.031 & 6229 & 23 & 3.933 & 0.044 \\
\hline $\mathrm{S} 3 \mathrm{C} 2$ & SDSS J214325.56-001037.6 & $1030-52914-177$ & 325.8565063 & -0.1771210 & -2.592 & 0.084 & 6154 & 56 & 4.049 & 0.120 \\
\hline $\mathrm{S} 3 \mathrm{C} 2$ & SDSS J214325.56-001037.6 & $1108-53227-176$ & 325.8565063 & -0.1771210 & -2.412 & 0.032 & 6167 & 35 & 4.005 & 0.062 \\
\hline $\mathrm{S} 3 \mathrm{C} 3$ & SDSS J003043.47-002056.2 & $1134-52644-284$ & 7.6811280 & -0.3489460 & -1.759 & 0.017 & 6055 & 31 & 4.359 & 0.104 \\
\hline $\mathrm{S} 3 \mathrm{C} 3$ & SDSS J003837.46-001822.8 & $1133-52993-070$ & 9.6560965 & -0.3063290 & -1.077 & & 5585 & 35 & 4.139 & 0.108 \\
\hline $\mathrm{S} 3 \mathrm{C} 3$ & SDSS J010042.57-010319.3 & $0395-51783-001$ & 15.1773758 & -1.0553480 & -1.209 & & 6029 & 45 & 3.793 & 0.096 \\
\hline $\mathrm{S} 3 \mathrm{C} 3$ & SDSS J010042.57-010319.3 & $1083-52520-050$ & 15.177 & -1.05 & -1.225 & & 589 & 26 & 3.718 & 0.077 \\
\hline $\mathrm{S} 3 \mathrm{C} 3$ & SDSS J010042.57-010319.3 & $1497-52886-049$ & 15.1773758 & -1.0553480 & -1.311 & 0.029 & 5889 & 36 & 3.735 & 0.103 \\
\hline $\mathrm{S} 3 \mathrm{C} 3$ & SDSS J080541.88+065800.1 & $2056-53463-483$ & 121.4245224 & 6.9667039 & -1.663 & 0.086 & 5276 & 44 & 4.152 & 0.203 \\
\hline $\mathrm{S} 3 \mathrm{C} 3$ & SDSS J083752.33+422801.9 & $0895-52581-531$ & 129.4680328 & 42.4672012 & -1.163 & 0.054 & 5621 & 31 & 4.316 & 0.074 \\
\hline $\mathrm{S} 3 \mathrm{C} 3$ & SDSS J101409.40+395431.0 & 1357-53034-194 & 153.5391846 & 39.9086037 & -1.739 & 0.020 & 5980 & 45 & 4.249 & 0.095 \\
\hline $\mathrm{S} 3 \mathrm{C} 3$ & SDSS J162108.52+010433.3 & 0346-51693-601 & 245.2855072 & 1.0759110 & -1.337 & 0.051 & 5727 & 46 & 4.432 & 0.061 \\
\hline $\mathrm{S} 3 \mathrm{C} 3$ & SDSS J213146.48+120942.4 & $1960-53289-574$ & 322.9436951 & 12.1617880 & -1.520 & 0.037 & 5616 & 27 & 3.501 & 0.162 \\
\hline
\end{tabular}

Notes. The last two entries in the bottom of the list are stars that appear in both " $\mathrm{S}_{3}$ " and "C2", and both " $\mathrm{S}_{3}$ " and "C3", respectively. The reason is that our selection criteria for "C2", "C3" and "S3" show some overlap. Also, we already noted in Section 7.4 and Section 7.6 that a connection between these streams probably exists.

Table 4

Putative Members of the Identified Streams: Photometry and Velocities

\begin{tabular}{|c|c|c|c|c|c|c|c|c|c|c|c|c|}
\hline STREAM & IAU NAME & $\begin{array}{c}u \\
(\mathrm{mag})\end{array}$ & $\begin{array}{c}g \\
(\mathrm{mag})\end{array}$ & $\begin{array}{c}r \\
(\mathrm{mag})\end{array}$ & $\begin{array}{c}i \\
(\mathrm{mag})\end{array}$ & $\begin{array}{c}z \\
(\mathrm{mag})\end{array}$ & $\begin{array}{c}d \\
(\mathrm{kpc})\end{array}$ & $\begin{array}{c}\sigma_{\mathrm{d}} \\
(\mathrm{kpc})\end{array}$ & $\begin{array}{c}v_{\mathrm{rad}} \\
\left(\mathrm{km} \mathrm{s}^{-1}\right)\end{array}$ & $\begin{array}{c}U \\
\left(\mathrm{~km} \mathrm{~s}^{-1}\right)\end{array}$ & $\begin{array}{c}V \\
\left(\mathrm{~km} \mathrm{~s}^{-1}\right)\end{array}$ & $\begin{array}{c}W \\
\left(\mathrm{~km} \mathrm{~s}^{-1}\right)\end{array}$ \\
\hline $\mathrm{C} 1$ & SDSS J003929.56+143550.1 & 16.845 & 15.809 & 15.329 & 15.132 & 15.116 & 0.708 & 0.044 & -110.1 & 50.3 & -25.0 & -57.6 \\
\hline $\mathrm{C} 1$ & SDSS J004044.06+143713.5 & 19.647 & 17.808 & 16.987 & 16.655 & 16.459 & 0.798 & 0.032 & -112.5 & 52.0 & -30.4 & -58.7 \\
\hline $\mathrm{C} 1$ & SDSS J011202.72+244140.1 & 18.173 & 17.193 & 16.721 & 16.543 & 16.501 & 1.421 & 0.080 & -152.8 & 115.7 & -4.9 & -57.8 \\
\hline $\mathrm{C} 1$ & SDSS J012737.07+385706.2 & 17.568 & 16.406 & 15.943 & 15.733 & 15.684 & 0.966 & 0.056 & -58.15 & -106.6 & -1.8 & -59.9 \\
\hline $\mathrm{C} 1$ & SDSS J012823.46+375742.5 & 20.032 & 18.645 & 18.039 & 17.814 & 17.738 & 1.933 & 0.114 & -95.7 & -52.6 & -14.5 & -62.5 \\
\hline $\mathrm{C} 1$ & SDSS J013249.36-091356.1 & 18.394 & 17.225 & 16.781 & 16.602 & 16.536 & 1.712 & 0.170 & 59.9 & -102.8 & -16.3 & -57.5 \\
\hline $\mathrm{C} 1$ & SDSS J014657.63-002829.4 & 20.847 & 19.088 & 18.343 & 18.044 & 17.879 & 1.854 & 0.107 & 49.2 & -110.2 & -2.3 & -47.8 \\
\hline $\mathrm{C} 1$ & SDSS J021054.90+224058.0 & 19.032 & 17.664 & 17.037 & 16.805 & 16.738 & 1.204 & 0.080 & 37.4 & -144.5 & -13.9 & -56.8 \\
\hline $\mathrm{C} 1$ & SDSS J023454.42+285631.8 & 17.75 & 16.792 & 16.363 & 16.194 & 16.101 & 1.397 & 0.084 & -151 & 106.3 & -11.2 & -47.9 \\
\hline $\mathrm{C} 1$ & SDSS J023736.38+261237.9 & 19.791 & 18.546 & 17.96 & 17.763 & 17.628 & 1.973 & 0.091 & 17.5 & -106.7 & -9.9 & -40.9 \\
\hline $\mathrm{C} 1$ & SDSS J024458.22-003204.3 & 19.081 & 17.962 & 17.467 & 17.276 & 17.189 & 1.763 & 0.124 & -14.35 & 86.1 & -11.3 & -52.1 \\
\hline $\mathrm{C} 1$ & SDSS J032649.09+054858.9 & 18.594 & 17.534 & 17.089 & 16.92 & 16.892 & 1.723 & 0.126 & 45.95 & -7.6 & -23.1 & -50.9 \\
\hline $\mathrm{C} 1$ & SDSS J060816.47+652237.7 & 18.791 & 17.651 & 17.139 & 16.938 & 16.862 & 1.615 & 0.064 & -32.5 & -117.6 & -21.4 & -49.3 \\
\hline $\mathrm{C} 1$ & SDSS J081851.60+010153.2 & 19.076 & 17.915 & 17.525 & 17.243 & 17.247 & 1.997 & 0.055 & 66.8 & 112.7 & -21.3 & -57.8 \\
\hline $\mathrm{C} 1$ & SDSS J084558.30+542552.5 & 17.475 & 16.569 & 16.212 & 16.068 & 16.037 & 1.513 & 0.103 & -112.7 & 32.6 & -6.9 & -68.4 \\
\hline $\mathrm{C} 1$ & SDSS J085520.23+105431.9 & 19.721 & 18.567 & 18.008 & 17.776 & 17.666 & 1.982 & 0.112 & 26.7 & 110.1 & -12.4 & -50.1 \\
\hline $\mathrm{C} 1$ & SDSS J094333.81+614255.3 & 19.271 & 17.862 & 17.207 & 16.94 & 16.808 & 1.195 & 0.075 & -217.1 & 171.2 & -20.1 & -38.5 \\
\hline $\mathrm{C} 1$ & SDSS J102023.87+484211.4 & 17.84 & 16.856 & 16.431 & 16.291 & 16.256 & 1.554 & 0.073 & 5 . & -133.2 & -26.6 & -47.4 \\
\hline $\mathrm{C} 1$ & SDSS J103806.73+452054.3 & 19.208 & 17.488 & 16.697 & 16.422 & 16.306 & 0.835 & 0.069 & 16.9 & -164.4 & -6.2 & -56.9 \\
\hline $\mathrm{C} 1$ & SDSS J113643.00+245755.1 & 18.347 & 17.336 & 16.907 & 16.757 & 16.72 & 1.856 & 0.130 & -18.6 & 20.9 & -15.8 & -53.8 \\
\hline $\mathrm{C} 1$ & SDSS J124629.08+285028.7 & 17.732 & 16.845 & 16.453 & 16.306 & 16.266 & 1.445 & 0.095 & -64.3 & 64.2 & -19.6 & -51.9 \\
\hline $\mathrm{C} 1$ & SDSS J124656.06+305644.2 & 17.078 & 16.096 & 15.66 & 15.504 & 15.415 & 0.946 & 0.058 & -70.1 & 121.0 & -24.5 & -45.9 \\
\hline $\mathrm{C} 1$ & SDSS J144844.46+254959.5 & 19.145 & 17.989 & 17.45 & 17.241 & 17.13 & 1.600 & 0.094 & -140.3 & -49.4 & -13.2 & -54.1 \\
\hline $\mathrm{C} 1$ & SDSS J150940.61+005724.1 & 17.844 & 16.901 & 16.522 & 16.387 & 16.397 & 1.895 & 0.107 & -30.7 & 51.7 & -19.8 & -66.4 \\
\hline $\mathrm{C} 1$ & SDSS J160819.86+522800.9 & 18.303 & 17.178 & 16.669 & 16.512 & 16.438 & 1.374 & 0.112 & -215.5 & 78.5 & -24.8 & -60.6 \\
\hline $\mathrm{C} 1$ & SDSS J162753.45+480813.1 & 17.027 & 16.162 & 15.818 & 15.703 & 15.693 & 1.292 & 0.083 & -222.8 & 36.8 & -32.7 & -59.8 \\
\hline $\mathrm{C} 1$ & SDSS J164228.88+371457.9 & 17.088 & 16.199 & 15.887 & 15.707 & 15.661 & 1.285 & 0.083 & -200.3 & 22.7 & -3.7 & -74.4 \\
\hline $\mathrm{C} 1$ & SDSS J164800.02+224725.9 & 17.822 & 16.941 & 16.589 & 16.468 & 16.415 & 1.777 & 0.120 & -177.2 & -13.2 & -3.4 & -56.7 \\
\hline $\mathrm{C} 1$ & SDSS J174211.76+245806.5 & 18.672 & 17.644 & 17.204 & 17 & 16.931 & 1.778 & 0.116 & -260 & -92.4 & -27.7 & -52.2 \\
\hline
\end{tabular}


Table 4

(Continued)

\begin{tabular}{|c|c|c|c|c|c|c|c|c|c|c|c|c|}
\hline STREAM & IAU NAME & $\begin{array}{c}u \\
(\mathrm{mag})\end{array}$ & $\begin{array}{c}g \\
(\mathrm{mag})\end{array}$ & $\begin{array}{c}r \\
(\mathrm{mag})\end{array}$ & $\begin{array}{c}i \\
(\mathrm{mag})\end{array}$ & $\begin{array}{c}z \\
(\mathrm{mag})\end{array}$ & $\begin{array}{c}d \\
(\mathrm{kpc})\end{array}$ & $\begin{array}{c}\sigma_{\mathrm{d}} \\
(\mathrm{kpc})\end{array}$ & $\begin{array}{c}v_{\text {rad }} \\
\left(\mathrm{km} \mathrm{s}^{-1}\right)\end{array}$ & $\begin{array}{c}U \\
\left(\mathrm{~km} \mathrm{~s}^{-1}\right)\end{array}$ & $\begin{array}{c}V \\
\left(\mathrm{~km} \mathrm{~s}^{-1}\right)\end{array}$ & $\begin{array}{c}W \\
\left(\mathrm{~km} \mathrm{~s}^{-1}\right)\end{array}$ \\
\hline$\overline{\mathrm{C} 1}$ & SDSS J233936.27+001812.8 & 19.056 & 17.801 & 17.24 & 17.039 & 16.911 & 1.476 & 0.102 & -89.3 & -82.3 & -25.9 & -47.6 \\
\hline $\mathrm{C} 1$ & SDSS J234150.16-011412.8 & 20.048 & 18.811 & 18.173 & 17.928 & 17.839 & 1.761 & 0.107 & -51.6 & -12.9 & -2.6 & -68.9 \\
\hline $\mathrm{C} 1$ & SDSS J235033.41+371115.7 & 17.652 & 16.706 & 16.308 & 16.149 & 16.133 & 1.502 & 0.096 & -154.9 & -102.8 & -25.5 & -48.6 \\
\hline $\mathrm{C} 2$ & SDSS J003315.18+083239.6 & 18.101 & 17.158 & 16.805 & 16.613 & 16.59 & 1.759 & 0.182 & -244.7 & & -93.5 & 76.9 \\
\hline $\mathrm{C} 2$ & SDSS J003335.38+064204.8 & 18.054 & 17.061 & 16.662 & 16.508 & 16.488 & 1.779 & 0.127 & -219.7 & 7.0 & -107.9 & 70.7 \\
\hline $\mathrm{C} 2$ & SDSS J003951.37+241507.1 & 18.137 & 17.259 & 16.908 & 16.777 & 16.764 & 1.975 & 0.113 & -248.6 & 37.7 & -74.9 & 64.1 \\
\hline $\mathrm{C} 2$ & SDSS J014003.43+224737.3 & 18.302 & 17.494 & 17.072 & 16.954 & 16.933 & 1.982 & 0.164 & -284.7 & 132.8 & -65.4 & 107.0 \\
\hline $\mathrm{C} 2$ & SDSS J024606.36-011303.6 & 19.279 & 17.883 & 17.227 & 16.982 & 16.849 & 1.222 & 0.066 & -19.95 & -57.6 & -109.6 & 64.1 \\
\hline $\mathrm{C} 2$ & SDSS J024606.36-011303.6 & 19.279 & 17.883 & 17.227 & 16.982 & 16.849 & 1.183 & 0.068 & -17.4 & -56.6 & -98.9 & 60.8 \\
\hline $\mathrm{C} 2$ & SDSS J061510.77+634737.4 & 18.381 & 17.327 & 16.866 & 16.664 & 16.636 & 1.561 & 0.072 & -233.9 & 151.7 & -78.2 & 59.4 \\
\hline $\mathrm{C} 2$ & SDSS J072805.35+363536.8 & 17.637 & 16.843 & 16.542 & 16.393 & 16.367 & 1.701 & 0.107 & 20.6 & 38.7 & -77.1 & 89.6 \\
\hline $\mathrm{C} 2$ & SDSS J072957.64+404615.9 & 18.502 & 17.462 & 17.037 & 16.857 & 16.793 & 1.887 & 0.114 & 111.4 & -84.0 & -61.2 & 82.1 \\
\hline $\mathrm{C} 2$ & SDSS J073626.44+215322.5 & 19.28 & 17.977 & 17.438 & 17.229 & 17.127 & 1.676 & 0.138 & 243 & -127.3 & -70.3 & 101.6 \\
\hline $\mathrm{C} 2$ & SDSS J074829.13+234423.8 & 19.036 & 17.906 & 17.4 & 17.189 & 17.152 & 1.842 & 0.114 & 263.6 & -167.7 & & 66.1 \\
\hline $\mathrm{C} 2$ & SDSS J075411.14+344532.4 & 17.121 & 16.2 & 15.895 & 15.775 & 15.7 & 1.268 & & & & & 69.8 \\
\hline $\mathrm{C} 2$ & SDSS J083939.81+064948.7 & 17.43 & 16.573 & 16.255 & 16.104 & 16.098 & 1.495 & 0.079 & 121.2 & 129.7 & -76.3 & 69.7 \\
\hline $\mathrm{C} 2$ & SDSS J084737.08+070118.2 & 18.739 & 17.19 & 16.569 & 16.277 & 16.147 & 0.964 & 0.063 & 269.8 & -101.9 & -79.9 & 49.1 \\
\hline $\mathrm{C} 2$ & SDSS J090533.11+432639.5 & 17.532 & 16.632 & 16.237 & 16.158 & 16.172 & 1.591 & 0.144 & 159.3 & -173.0 & -59.9 & 50.1 \\
\hline $\mathrm{C} 2$ & SDSS J091720.56+020254.3 & 17.408 & 16.571 & 16.185 & 16.056 & 16.027 & 1.448 & 0.066 & 320 & -175.3 & -64.9 & 68.7 \\
\hline $\mathrm{C} 2$ & SDSS J092013.59+284917.4 & 17.008 & 16.197 & 15.9 & 15.765 & 15.777 & 1.377 & 0.092 & 128.2 & -14.2 & -77.0 & 66.5 \\
\hline $\mathrm{C} 2$ & SDSS J092213.39+225504.1 & 19.352 & 17.699 & 17.051 & 16.748 & 16.629 & 1.206 & 0.093 & 259.5 & -150.6 & -74.2 & 88.0 \\
\hline $\mathrm{C} 2$ & SDSS J093632.83+473706.4 & 17.65 & 16.745 & 16.386 & 16.276 & 16.237 & 1.722 & 0.117 & -22.2 & 68.0 & -99.7 & 72.1 \\
\hline $\mathrm{C} 2$ & SDSS J094018.33-003356.5 & 18.044 & 17.169 & 16.816 & 16.657 & 16.632 & 1.846 & 0.123 & 286.5 & -127.1 & -65.6 & 58.0 \\
\hline $\mathrm{C} 2$ & SDSS J095506.81+114300.7 & 16.954 & 15.984 & 15.606 & 15.479 & 15.474 & 1.219 & 0.105 & 181 & 33.5 & -75 & 67.1 \\
\hline $\mathrm{C} 2$ & SDSS J095908.86+005645.0 & 18.673 & 17.697 & 17.273 & 17.092 & 17.012 & 1.935 & 0.143 & 255.8 & -28.7 & & 63.5 \\
\hline $\mathrm{C} 2$ & SDSS J101455.05+253710.0 & 19.442 & 18.033 & 17.534 & 17.281 & 17.166 & 1.794 & 0.1 & & -127.4 & & 72.8 \\
\hline $\mathrm{C} 2$ & SDSS J104621.93+004321.8 & 18.732 & 17.526 & 17.035 & 16.848 & 16.749 & 1.551 & & & & & 80.7 \\
\hline $\mathrm{C} 2$ & SDSS J105039.60-004524.8 & 19.866 & 18.249 & 17.527 & 17.244 & 17.1 & 1.260 & 0.0 & 265.4 & -188.8 & & 69.7 \\
\hline $\mathrm{C} 2$ & SDSS J105338.83+481716.5 & 19.716 & 18.422 & 17.792 & 17.508 & 17.368 & 1.337 & 0.053 & 79.8 & -164.7 & -66.6 & 50.4 \\
\hline $\mathrm{C} 2$ & SDSS J105649.28+014446.2 & 18.816 & 17.806 & 17.358 & 17.143 & 17.079 & 1.788 & 0.127 & 247.1 & 8.4 & -97.4 & 83.5 \\
\hline $\mathrm{C} 2$ & SDSS J111101.99+103838.3 & 19.354 & 18.097 & 17.517 & 17.29 & 17.33 & 1.641 & 0.148 & 167.4 & 169.8 & -76.2 & 87.8 \\
\hline $\mathrm{C} 2$ & SDSS J113811.21+482056.1 & 18.549 & 17.557 & 17.104 & 16.934 & 16.937 & 1.990 & 0.143 & 45.1 & -132.1 & -61.4 & 59.2 \\
\hline $\mathrm{C} 2$ & SDSS J115028.60+015703.2 & 17.476 & 16.629 & 16.263 & 16.125 & 16.06 & 1.419 & 0.095 & 202.2 & 35.1 & -90.2 & 62.8 \\
\hline $\mathrm{C} 2$ & SDSS J122249.57+010247.7 & 18.865 & 17.033 & 16.269 & 15.988 & 15.801 & 0.727 & 0.061 & 199.2 & 114.0 & -92.1 & 60.8 \\
\hline $\mathrm{C} 2$ & SDSS J124601.97+285517.0 & 17.059 & 16.194 & 15.842 & 15.733 & 15.737 & 1.351 & 0.097 & 70.25 & -112.1 & -68.6 & 77.5 \\
\hline $\mathrm{C} 2$ & SDSS J152938.97+483843.7 & 18.045 & 17.067 & 16.794 & 16.61 & 16.573 & 1.890 & 0.224 & -145 & 9.9 & -104.8 & 72.3 \\
\hline $\mathrm{C} 2$ & SDSS J161143.97+534352.6 & 19.465 & 17.707 & 16.894 & 16.585 & 16.458 & 0.831 & 0.023 & -180.1 & 143.7 & & 50.4 \\
\hline $\mathrm{C} 2$ & SDSS J161513.53+070012.5 & 16.542 & 15.658 & 15.332 & 15.207 & 15.155 & 1.003 & 0.056 & -31.95 & 35.4 & -100.9 & 75.5 \\
\hline $\mathrm{C} 2$ & SDSS J163043.32+633133.6 & 18.503 & 17.143 & 16.608 & & & 1.143 & & -189.9 & & & 75.0 \\
\hline $\mathrm{C} 2$ & SDSS J172 & 21.473 & 19.181 & 18.201 & 17.845 & 17.655 & 1.066 & 0.025 & $-1 c$ & -12.1 & & 59.9 \\
\hline $\mathrm{C} 2$ & SDSS J220031.01+001657.2 & 18.092 & 16.612 & 15.991 & 15.775 & 15.682 & 0.802 & 0.044 & -163.2 & 173.5 & -49.3 & 73.2 \\
\hline $\mathrm{C} 2$ & SDSS J224514.28-003350.8 & 19.582 & 18.27 & 17.688 & 17.395 & 17.275 & 1.437 & 0.061 & -172.9 & 167.9 & -52.1 & 59.1 \\
\hline $\mathrm{C} 2$ & SDSS J224738.34+234631.6 & 19.528 & 18.587 & 18.049 & 17.807 & 17.864 & 1.981 & 0.227 & -309.7 & -23.3 & -79.9 & 101.3 \\
\hline $\mathrm{C} 2$ & SDSS J233651.29+462505.0 & 18.4 & 17.417 & 17.003 & 16.795 & 16.786 & 1.775 & 0.114 & -335.9 & 128.2 & -79.2 & 86.2 \\
\hline $\mathrm{C} 2$ & SDSS J233819.91+000557.3 & 18.492 & 17.555 & 17.102 & 16.9 & 16.847 & 1.501 & 0.101 & -214.2 & -127.8 & -89.5 & 58.4 \\
\hline $\mathrm{C} 2$ & SDSS J233835.27+075729.9 & 18.685 & 17.534 & 17.038 & 16.829 & 16.725 & 1.482 & 0.093 & -245.7 & -6.1 & -61.9 & 91.6 \\
\hline $\mathrm{C} 2$ & SDSS J234345.10+002735.9 & 20.884 & 19.152 & 18.333 & 18.006 & 17.826 & 1.500 & 0.044 & -200 & -165.4 & -79.9 & 53.5 \\
\hline $\mathrm{C} 3$ & SDSS J003149.73-004244.2 & 19.951 & 18.106 & 17.405 & 17.121 & 16.948 & 1.349 & 0.056 & -225.4 & 40.1 & -10.7 & 144.0 \\
\hline $\mathrm{C} 3$ & SDSS J003542.54+061441.9 & 15.83 & 14.652 & 14.174 & 14.019 & 13.909 & 0.462 & 0.039 & -207.5 & -73.5 & -35 & 126.8 \\
\hline $\mathrm{C} 3$ & SDSS J003609.72-004756.4 & 17.9 & & & & & & & -229 & 77 & -3 & 133.8 \\
\hline $\mathrm{C} 3$ & SDSS J003947.76+003919.3 & 18.479 & 17.432 & 16.99 & 16.765 & 16.706 & 1.435 & 0.085 & -204.9 & -38.1 & -24.2 & 133.9 \\
\hline $\mathrm{C} 3$ & SDSS J003947.76+003919.3 & 18.479 & 17.432 & 16.99 & 16.765 & 16.706 & 1.406 & 0.097 & -210.7 & -34.9 & -23.2 & 140.2 \\
\hline $\mathrm{C} 3$ & SDSS J003947.76+003919.3 & 18.479 & 17.432 & 16.99 & 16.765 & 16.706 & 1.417 & 0.079 & -209.6 & -35.9 & -24.0 & 138.8 \\
\hline $\mathrm{C} 3$ & SDSS J004647.08+143012.0 & 17.39 & 16.474 & 16.133 & 15.974 & 15.954 & 1.409 & 0.111 & -244.3 & 90.1 & -4.6 & 123.3 \\
\hline $\mathrm{C} 3$ & SDSS J012450.36+074506.9 & 18.715 & 17.674 & 17.205 & 17.045 & 16.972 & 1.936 & 0.106 & -184.6 & -36.0 & -21.3 & 141.0 \\
\hline $\mathrm{C} 3$ & SDSS J014710.34+145458.9 & 18.969 & 17.748 & 17.229 & 17.015 & 16.965 & 1.640 & 0.122 & -269.8 & 179.6 & -17.8 & 110.4 \\
\hline $\mathrm{C} 3$ & SDSS J021022.12+214531.8 & 17.186 & 16.138 & 15.625 & 15.444 & 15.347 & 0.760 & 0.039 & -283.4 & 167.1 & -20.1 & 127.5 \\
\hline $\mathrm{C} 3$ & SDSS J022958.15-075952.6 & 18.252 & 17.291 & 16.803 & 16.624 & 16.558 & 1.325 & 0.072 & -138.7 & 98.8 & -9.2 & 114.0 \\
\hline $\mathrm{C} 3$ & SDSS J032120.59+060355.3 & 17.996 & 16.927 & 16.396 & 16.188 & 16.165 & 0.956 & 0.042 & -9.95 & -87.1 & -4.8 & 120.9 \\
\hline $\mathrm{C} 3$ & SDSS J081525.99+362755.8 & 16.983 & 16.069 & 15.684 & 15.523 & 15.494 & 0.986 & 0.038 & 193.3 & -112.4 & -54.9 & 130.3 \\
\hline $\mathrm{C} 3$ & SDSS J082533.57+182230.9 & 17.006 & 16.184 & 15.906 & 15.773 & 15.784 & 1.406 & 0.141 & 243.9 & -111.4 & -12.3 & 121.2 \\
\hline $\mathrm{C} 3$ & SDSS J084043.12+095140.2 & 17.733 & 16.952 & 16.584 & 16.444 & 16.358 & 1.625 & 0.107 & 217.5 & -2.6 & -56.3 & 129.2 \\
\hline $\mathrm{C} 3$ & SDSS J092032.03+231836.5 & 17.562 & 16.782 & 16.417 & 16.316 & 16.319 & 1.747 & 0.148 & 253.9 & -143.7 & -9.5 & 119.2 \\
\hline
\end{tabular}


Table 4

(Continued)

\begin{tabular}{|c|c|c|c|c|c|c|c|c|c|c|c|c|}
\hline STREAM & IAU NAME & $\begin{array}{c}u \\
(\mathrm{mag})\end{array}$ & $\begin{array}{c}g \\
(\mathrm{mag}) \\
\end{array}$ & $\begin{array}{c}r \\
(\mathrm{mag}) \\
\end{array}$ & $\begin{array}{c}i \\
(\mathrm{mag}) \\
\end{array}$ & $\begin{array}{c}z \\
(\mathrm{mag})\end{array}$ & $\begin{array}{c}d \\
(\mathrm{kpc})\end{array}$ & $\begin{array}{c}\sigma_{\mathrm{d}} \\
(\mathrm{kpc})\end{array}$ & $\begin{array}{c}v_{\text {rad }} \\
\left(\mathrm{km} \mathrm{s}^{-1}\right)\end{array}$ & $\begin{array}{c}U \\
\left(\mathrm{~km} \mathrm{~s}^{-1}\right) \\
\end{array}$ & $\begin{array}{c}V \\
\left(\mathrm{~km} \mathrm{~s}^{-1}\right) \\
\end{array}$ & $\begin{array}{c}W \\
\left(\mathrm{~km} \mathrm{~s}^{-1}\right) \\
\end{array}$ \\
\hline C3 & SDSS J092824.65+064514.5 & 19.166 & 17.633 & 16.888 & 16.612 & 16.491 & 0.855 & 0.040 & 169.8 & 106.3 & -14.5 & 141.7 \\
\hline $\mathrm{C} 3$ & SDSS J094030.57+381706.9 & 17.43 & 16.608 & 16.238 & 16.122 & 16.137 & 1.380 & 0.075 & 64.6 & 79.7 & -32.3 & 131.0 \\
\hline $\mathrm{C} 3$ & SDSS J094934.58+001711.5 & 17.74 & 16.853 & 16.522 & 16.403 & 16.38 & 1.951 & 0.158 & 281.7 & -118.8 & -17.1 & 116.4 \\
\hline $\mathrm{C} 3$ & SDSS J100406.88+060336.0 & 18.322 & 17.471 & 17.1 & 16.927 & 16.868 & 1.849 & 0.141 & 281.8 & -117.5 & -27.5 & 126.4 \\
\hline $\mathrm{C} 3$ & SDSS J100908.52+013225.0 & 16.839 & 16.019 & 15.686 & 15.55 & 15.512 & 1.095 & 0.085 & 227.3 & 49.1 & -21.2 & 132.3 \\
\hline $\mathrm{C} 3$ & SDSS J101140.34+354038.8 & 19.622 & 18.046 & 17.298 & 16.974 & 16.82 & 0.894 & 0.019 & 200.3 & -128.8 & -20.7 & 127.2 \\
\hline $\mathrm{C} 3$ & SDSS J101749.41+404601.6 & 19.029 & 17.96 & 17.462 & 17.263 & 17.149 & 1.619 & 0.094 & 61 & 67.5 & -10.7 & 118.9 \\
\hline $\mathrm{C} 3$ & SDSS J103459.70+082335.9 & 17.285 & 16.431 & 16.074 & 15.964 & 15.933 & 1.472 & 0.110 & 257.9 & -82.8 & -37.5 & 124.6 \\
\hline $\mathrm{C} 3$ & SDSS J103658.20+163609.3 & 19.262 & 18.701 & 18.132 & 17.969 & 17.935 & 1.985 & 0.214 & 219.5 & -33.1 & -7.5 & 141.7 \\
\hline C3 & SDSS J104827.81+005524.0 & 19.054 & 17.716 & 17.197 & 17.012 & 16.898 & 1.844 & 0.106 & 261.9 & -103.5 & -4.0 & 137.3 \\
\hline $\mathrm{C} 3$ & SDSS J104838.61+005839.4 & 17.467 & 16.663 & 16.3 & 16.184 & 16.212 & 1.539 & 0.150 & 227 & 11.2 & -1.0 & 126.3 \\
\hline $\mathrm{C} 3$ & SDSS J105236.88-004816.8 & 19.407 & 18.166 & 17.642 & 17.427 & 17.409 & 1.989 & 0.136 & 271.7 & -69.0 & -25.5 & 141.5 \\
\hline $\mathrm{C} 3$ & SDSS J105643.51+480559.6 & 20.535 & 18.923 & 18.319 & 18.068 & 17.983 & 1.960 & 0.128 & 74.9 & -18.1 & -23.9 & 121.4 \\
\hline $\mathrm{C} 3$ & SDSS J124923.70+291857.5 & 17.79 & 16.82 & 16.437 & 16.254 & 16.252 & 1.550 & 0.104 & 104.8 & -162.3 & -47.0 & 114.7 \\
\hline $\mathrm{C} 3$ & SDSS J160840.68+524221.9 & 18.45 & 17.345 & 16.871 & & & 1.538 & 0.078 & -99 & & & 123.4 \\
\hline $\mathrm{C} 3$ & SDSS J161247.16+194440.3 & 17.582 & 16.791 & 16.463 & 16.345 & 16.289 & 1.739 & 0.126 & -28.6 & 13.1 & -34.1 & 123.7 \\
\hline $\mathrm{C} 3$ & SDSS J171058.39+430326.4 & 20.343 & 18.35 & 17.408 & 17.03 & 16.819 & 0.781 & 0.022 & -90.1 & 76.7 & -15.4 & 130.8 \\
\hline $\mathrm{C} 3$ & SDSS J172433.72+260332.6 & 19.787 & 18.311 & 17.585 & 17.305 & 17.161 & 1.078 & 0.031 & -101.4 & 12.4 & & 125.8 \\
\hline $\mathrm{C} 3$ & SDSS J204435.17-005117.0 & 17.366 & 16.374 & 15.913 & 15.767 & 15.664 & 0.979 & 0.029 & -317.8 & -184.0 & -10 & 106.3 \\
\hline $\mathrm{C} 3$ & SDSS J233626.78+100057.5 & 18.29 & 17.188 & 16.708 & 16.508 & 16.413 & 1.433 & 0.156 & -262.3 & 83.3 & & 142.8 \\
\hline $\mathrm{C} 3$ & SDSS J233912.75-004742.7 & 16.962 & 16.075 & 15.722 & 15.571 & 15.563 & 1.102 & 0.080 & -240.4 & -8.6 & -34.0 & 129.9 \\
\hline $\mathrm{C} 4$ & SDSS J011441.01+010914.8 & 17.393 & 16.515 & 16.125 & 15.997 & 15.905 & 1.193 & 0.118 & 31.4 & -40.0 & 179.6 & -26.2 \\
\hline $\mathrm{C} 4$ & SDSS J023158.11+255029.1 & 17.304 & 16.462 & 16.044 & 15.936 & 15.889 & 1.176 & 0.070 & 20.3 & 1.0 & 162.1 & -68.6 \\
\hline $\mathrm{C} 4$ & SDSS J023435.85-084755.9 & 17.447 & 16.566 & 16.224 & 16.133 & 16.099 & 1.616 & 0.171 & 62.6 & -24.4 & 160.5 & -43.9 \\
\hline $\mathrm{C} 4$ & SDSS J024418.33-000947.9 & 17.013 & 16.067 & 15.712 & 15.597 & 15.563 & 1.061 & 0.080 & -14.05 & 29.8 & 183.0 & 6.0 \\
\hline $\mathrm{C} 4$ & SDSS J081918.72+390006.5 & 19.515 & 18.108 & 17.576 & 17.397 & 17.367 & 1.582 & 0.089 & -37.9 & 41.7 & 172.6 & -18.3 \\
\hline $\mathrm{C} 4$ & SDSS J103649.93+121219.8 & 16.452 & 15.58 & 15.248 & & 15.085 & 0.880 & & & & & -36.6 \\
\hline $\mathrm{C} 4$ & SDSS J105230.56+314419.2 & 17.532 & 16.631 & 16.268 & 16.135 & 16.069 & 1.396 & 0.100 & -29.9 & 32.7 & 182.3 & -21.5 \\
\hline $\mathrm{C} 4$ & SDSS J105424.31+372221.2 & 18.669 & 17.667 & 17.175 & 16.974 & 16.891 & 1.392 & 0.065 & -23 & -3.6 & 176.1 & -27.0 \\
\hline $\mathrm{C} 4$ & SDSS J113626.47+020433.3 & 17.444 & 16.59 & 16.286 & 16.135 & 16.039 & 1.552 & 0.118 & -38 & 67. & 177 & -62.4 \\
\hline $\mathrm{C} 4$ & SDSS J124342.45+101708.0 & 17.175 & 16.307 & 15.964 & 15.779 & 15.707 & 1.101 & 0.070 & -27.5 & 27.9 & 158.0 & -42.2 \\
\hline $\mathrm{C} 4$ & SDSS J124615.79-090738.0 & 20.739 & 19.415 & 18.747 & 18.442 & 18.307 & 1.988 & 0.073 & & 11.6 & 159.9 & -47.3 \\
\hline $\mathrm{C} 4$ & SDSS J142255.29+125353.1 & 16.902 & 16.075 & 15.766 & 15.626 & 15.629 & 1.189 & 0.059 & -8.5 & 66.2 & 179.6 & -27.3 \\
\hline $\mathrm{C} 4$ & SDSS J143149.30+243552.0 & 18.603 & 17.571 & 17.179 & 17.027 & 16.983 & 1.876 & 0.147 & -32.4 & 50.2 & 167.4 & -28.8 \\
\hline $\mathrm{C} 4$ & SDSS J151555.14+135708.8 & 17.522 & 16.656 & 16.338 & 16.217 & 16.214 & 1.569 & 0.109 & 7 & 63.0 & 188.0 & -11.2 \\
\hline $\mathrm{C} 4$ & SDSS J163713.47+632122.9 & 18.845 & 17.223 & 16.6 & 16.43 & 16.292 & 0.897 & 0.050 & -53.7 & 27.8 & 168.1 & -5.8 \\
\hline $\mathrm{C} 4$ & SDSS J174259.25+244823.2 & 19.035 & 17.94 & 17.46 & 17.24 & 17.152 & 1.534 & 0.086 & -66.4 & -21.7 & 178.8 & -29.8 \\
\hline $\mathrm{C} 4$ & SDSS J200336.15+600006.9 & 17.922 & 15.798 & 15.035 & 14.784 & 14.675 & 0.325 & 0.005 & -50.1 & 24.5 & 177.2 & -3.5 \\
\hline $\mathrm{C} 4$ & SDSS J211011.44+002324.9 & 17.282 & 16.24 & 15.715 & 15.512 & 15.447 & 0.672 & 0.026 & & & & -55.2 \\
\hline $\mathrm{C} 4$ & SDSS J215433.88-071000.4 & 16.756 & 15.911 & 15.572 & 15.438 & 15.3 & 1.04 & 0.0 & & -56 & .7 & -39.0 \\
\hline $\mathrm{C} 4$ & SDSS J234017.29-003337.6 & 17.895 & 16.573 & 16.051 & 15.861 & 15.804 & 0.788 & 0.031 & -11.3 & 61.0 & 176.6 & -8.5 \\
\hline H99 & SDSS J004500.91+134546.1 & 17.999 & 17.122 & 16.724 & 16.587 & 16.528 & 1.550 & 0.117 & 173.4 & -9.2 & 183.0 & -244.7 \\
\hline H99 & SDSS J004606.09+005812.0 & 18.223 & 17.114 & 16.648 & 16.475 & 16.417 & 1.466 & 0.107 & 150.7 & 98.0 & 170.3 & -213.1 \\
\hline H99 & SDSS J004755.30+143355.6 & 19.062 & 17.865 & 17.266 & 17.019 & 16.877 & 1.236 & 0.071 & 140.6 & 21.1 & 156.6 & -237.9 \\
\hline H99 & SDSS J012316.16-001508.5 & 18.753 & 17.818 & 17.341 & 17.144 & 17.102 & 1.594 & 0.105 & 199.4 & 40.9 & 144.0 & -258.3 \\
\hline H99 & SDSS J014552.75+134648.5 & 18.248 & 17.193 & 16.659 & 16.434 & 16.339 & 1.012 & 0.056 & 161.6 & 5.9 & 143.8 & -255.8 \\
\hline H99 & SDSS J014858.66+001851.5 & 19.806 & 18.348 & 17.689 & 17.428 & 17.302 & 1.347 & 0.074 & 201.2 & 4.7 & 146.2 & -245.4 \\
\hline H99 & SDSS J015218.06-003227.2 & 19.016 & 17.602 & 16.974 & 16.738 & 16.663 & 1.254 & 0.060 & 201.5 & 13.1 & 153.1 & -245.6 \\
\hline H99 & SDSS J021139.03+060824.3 & 18.527 & 17.468 & 16.954 & 16.748 & 16.688 & 1.331 & 0.067 & 177.3 & 50.4 & 153.9 & -271.1 \\
\hline H99 & SDSS J022207.13+011345.2 & 17.876 & 16.733 & 16.17 & 15.981 & 15.868 & 0.836 & 0.053 & 203.18333 & 19.3 & 147.7 & -264.1 \\
\hline H99 & SDSS J081654.87+265422.0 & 16.326 & 15.514 & 15.165 & 15.038 & 15.05 & 0.893 & 0.048 & -109.5 & 11.2 & 159.2 & -245.4 \\
\hline H99 & SDSS J092940.69+410552.2 & 16.417 & 15.544 & 15.241 & 15.106 & 15.082 & 0.995 & 0.102 & -215.8 & 31.7 & 110.7 & -272.4 \\
\hline H99 & SDSS J101829.06+650218.9 & 17.452 & 16.552 & 16.151 & 16.069 & 16.021 & 1.434 & 0.113 & -278.4 & 67.5 & 122.6 & -280.9 \\
\hline H99 & SDSS J103611.02+131457.9 & 17.782 & 16.979 & 16.65 & 16.533 & 16.489 & 1.897 & 0.111 & -181.5 & -22.0 & 145.3 & -269.4 \\
\hline H99 & SDSS J125635.48+403725.3 & 17.742 & 16.86 & 16.523 & 16.388 & 16.36 & 1.825 & 0.163 & -224 & 2.2 & 192.6 & -217.3 \\
\hline H99 & SDSS J163516.45+143604.5 & 18.338 & 17.466 & 17.066 & 16.92 & 16.888 & 1.733 & 0.078 & -149.6 & 32.5 & 184.1 & -239.3 \\
\hline H99 & SDSS J172312.11+275319.5 & 16.936 & 15.826 & 15.309 & 15.122 & 15.073 & 0.628 & 0.033 & -170.4 & 68.7 & 136.1 & -270.5 \\
\hline H99 & SDSS J211224.90+002453.8 & 17.287 & 16.491 & 16.168 & 16.073 & 16.046 & 1.640 & 0.083 & 78.1 & 13.6 & 118.1 & -284.8 \\
\hline H99 & SDSS J211224.91+002453.8 & 17.287 & 16.491 & 16.168 & 16.073 & 16.046 & 1.627 & 0.086 & 82.12 & 16.0 & 122.2 & -284.7 \\
\hline H99 & SDSS J222048.55+132527.1 & 18.432 & 17.371 & 16.947 & 16.792 & 16.725 & 1.815 & 0.105 & 96.8 & 107.6 & 143.4 & -241.3 \\
\hline H99 & SDSS J224938.70-083003.6 & 18.356 & 17.261 & 16.814 & 16.671 & 16.625 & 1.861 & 0.106 & 203.9 & 80.8 & 205.9 & -227.8 \\
\hline H99 & SDSS J234742.49+000253.1 & 17.74 & 16.806 & 16.417 & 16.248 & 16.201 & 1.566 & 0.111 & 181.4 & -94.1 & 107.0 & -274.3 \\
\hline
\end{tabular}


Table 4

(Continued)

\begin{tabular}{|c|c|c|c|c|c|c|c|c|c|c|c|c|}
\hline STREAM & IAU NAME & $\begin{array}{c}u \\
\text { (mag) }\end{array}$ & $\begin{array}{c}g \\
\text { (mag) } \\
\end{array}$ & $\begin{array}{c}r \\
(\mathrm{mag}) \\
\end{array}$ & $\begin{array}{c}i \\
(\mathrm{mag}) \\
\end{array}$ & $\begin{array}{c}z \\
(\mathrm{mag})\end{array}$ & $\begin{array}{c}d \\
(\mathrm{kpc})\end{array}$ & $\begin{array}{c}\sigma_{\mathrm{d}} \\
(\mathrm{kpc})\end{array}$ & $\begin{array}{c}v_{\text {rad }} \\
\left(\mathrm{km} \mathrm{s}^{-1}\right)\end{array}$ & $\begin{array}{c}U \\
\left(\mathrm{~km} \mathrm{~s}^{-1}\right) \\
\end{array}$ & $\begin{array}{c}V \\
\left(\mathrm{~km} \mathrm{~s}^{-1}\right) \\
\end{array}$ & $\begin{array}{c}W \\
\left(\mathrm{~km} \mathrm{~s}^{-1}\right) \\
\end{array}$ \\
\hline RAVE & SDSS J013839.30-093045.6 & 18.762 & 17.655 & 17.127 & 16.964 & 16.859 & 1.606 & 0.102 & -82.4 & -61.8 & 56.5 & 97.2 \\
\hline RAVE & SDSS J014203.92-090422.8 & 18.646 & 17.313 & 16.802 & 16.656 & 16.551 & 1.649 & 0.144 & -136.4 & & & 115.9 \\
\hline RAVE & SDSS J015905.74-003222.3 & 19.221 & 18.192 & 17.691 & 17.499 & 17.421 & 1.914 & 0.133 & -118.1 & 40.1 & & 91.0 \\
\hline RAVE & SDSS J033942.37-060709.4 & 19.042 & 17.363 & 16.661 & 16.397 & 16.288 & 1.011 & 0.064 & 18.9 & -57.4 & 77.5 & 82.0 \\
\hline RAVE & SDSS J082021.79+391100.7 & 18.864 & 17.739 & 17.207 & 16.989 & 16.905 & 1.411 & 0.094 & 79.1 & -29.2 & 79.0 & 79.7 \\
\hline RAVE & SDSS J085211.24+372747.5 & 18.707 & 17.627 & 17.127 & 16.971 & 16.918 & 1.984 & 0.153 & 103.3 & -27.1 & 48.9 & 101.7 \\
\hline RAVE & SDSS J095955.31-002950.1 & 18.681 & 17.519 & 17.048 & 16.858 & 16.752 & 1.685 & 0.106 & 180 & -47.2 & 75.5 & 98.7 \\
\hline RAVE & SDSS J103554.79+454500.5 & 19.487 & 18.355 & 17.815 & 17.562 & 17.52 & 1.729 & 0.130 & 92.7 & -29.7 & 32.6 & 113.4 \\
\hline RAVE & SDSS J103739.10+383729.0 & 18.388 & 17.214 & 16.672 & 16.434 & 16.356 & 1.123 & 0.066 & 97.8 & -18.9 & 52.8 & 98.3 \\
\hline RAVE & SDSS J111311.63-164457.4 & 17.117 & 16.199 & 15.821 & 15.671 & 15.626 & 1.166 & 0.072 & 176.1 & -23.5 & 70.6 & 99.0 \\
\hline RAVE & SDSS J111434.17+094641.6 & 17.686 & 16.196 & 15.548 & 15.341 & 15.3 & 0.659 & 0.050 & 149.9 & 39.9 & 68.4 & 105.9 \\
\hline RAVE & SDSS J122210.68+002445.2 & 20.363 & 18.655 & 17.925 & 17.666 & 17.482 & & & 156.3 & 45.1 & & 100.6 \\
\hline RAVE & SDSS J123456.17+515326.2 & 18.722 & & 17.032 & & & & & 38.5 & & & 9.8 \\
\hline RAVE & SDSS J132647.54+295741.2 & 19.199 & 18.169 & 17.68 & 17.465 & 17.434 & 1.819 & 0.117 & 59.2 & -37.6 & 84.8 & 87.8 \\
\hline RAVE & SDSS J154838.74+445401.8 & 16.761 & 15.899 & 15.529 & 15.428 & 15.402 & 1.161 & 0.073 & -23.9 & 39.5 & 55.9 & 101.7 \\
\hline RAVE & SDSS J212730.72+103653.3 & 17.878 & 16.667 & 16.153 & 15.989 & 15.918 & 1.107 & 0.072 & -184.9 & -15.4 & 54.3 & 90.8 \\
\hline RAVE & SDSS J224018.33-000629.9 & 18.206 & 17.102 & 16.582 & 16.388 & 16.325 & 1.178 & 0.079 & -171.4 & & 48.1 & 102.3 \\
\hline RAVE & SDSS J224616.22+001513.5 & 17.428 & 16.419 & 15.987 & 15.762 & 15.718 & 1.039 & 0.054 & -165.6 & 22.0 & 57.6 & 92.6 \\
\hline RAVE & SDSS J225744.52+061315.4 & 17.105 & 15.917 & 15.409 & 15.189 & 15.11 & 0.708 & 0.043 & -203.1 & -0.4 & 31.0 & 104.4 \\
\hline S3 & SDSS J014400.30+231504.1 & 18.543 & 17.495 & 16.981 & 16.774 & 16.676 & 1.352 & 0.072 & -151.7 & -86.9 & & 108.6 \\
\hline S3 & SDSS J025220.13+335550.4 & 17.326 & 16.299 & 15.839 & 15.682 & 15.636 & 1.007 & 0.053 & -33.6 & -132.7 & & 96.7 \\
\hline S3 & SDSS J035419.29-045941.0 & 17.211 & 16.268 & 15.896 & 15.763 & 15.778 & 1.469 & 0.093 & -118.8 & 171.5 & -39 & 89.7 \\
\hline S3 & SDSS J063233.61+273645.5 & 19.76 & 18.389 & 17.672 & 17.455 & 17.295 & 1.274 & 0.041 & 173.9 & -118.6 & -53.3 & 98.3 \\
\hline S3 & SDSS J071848.45+313202.6 & 19.187 & 17.994 & 17.531 & 17.227 & 17.135 & 1.990 & & 215 & -156.6 & & 80.9 \\
\hline S3 & SDSS J072100.85+411425.4 & 16.833 & 15.797 & 15.3 & 15.243 & & & & & & & \\
\hline S3 & SDSS J073614.44+411329.5 & 17.405 & 16.369 & 15.959 & 15.817 & 15.7 & 1.225 & 0.0 & -12 & 62 & -4 & 100.1 \\
\hline S3 & 403.2 & 18.282 & 17.279 & 16.799 & 16.6 & 16.4 & 1.365 & 0.0 & & 60 & -5 & 102.6 \\
\hline S3 & $.49+393309.6$ & 18.056 & 17.082 & 16.684 & 16.5 & 16.417 & 1.618 & 0.110 & -2.2 & 108 & -50 & 95.7 \\
\hline S3 & SDSS J092513.14+071055.3 & 19.042 & 17.683 & 17.07 & 16.789 & 16.697 & 1.039 & 0.046 & 303.6 & -187.4 & -28.2 & 88.7 \\
\hline S3 & SDSS J111847.11+090301.0 & 19.593 & 18.237 & 17.628 & 17.404 & 17.329 & 1.896 & 0.070 & 220.5 & -42.0 & -52.7 & 107.5 \\
\hline S3 & SDSS J170051.55+390528.4 & 16.809 & 15.751 & 15.333 & 15.132 & 15.08 & 0.814 & 0.066 & -88.9 & 135.0 & -3 & 95.2 \\
\hline S3 & SDSS J180538.01+241532.1 & 19.25 & 17.712 & 16.962 & 16.65 & 16.511 & 0.839 & 0.033 & -75.4 & 153.4 & -34.0 & 96.4 \\
\hline S3 & SDSS J215703.65+003723.0 & 20.247 & 18.72 & 17.934 & 17.579 & 17.36 & 1.109 & 0.034 & -180.2 & 160.7 & -51.2 & 90.9 \\
\hline S3 & SDSS J224417.24+001749.1 & 18.236 & 16.323 & 15.453 & 15.098 & 14.923 & 0.360 & 0.011 & -187.5 & 144.3 & -22.4 & 91.3 \\
\hline S3 & SDSS J224650.01+004000.4 & 19.254 & 17.516 & 16.738 & 16.436 & 16.299 & 0.882 & 0.042 & -226.9 & 76.3 & -45.3 & 103.1 \\
\hline S3 & SDSS J230110.77+054925.9 & 17.747 & 16.999 & 16.642 & 16.52 & & & 0.162 & -238.4 & 70 & & 101.7 \\
\hline $\mathrm{S} 3 \mathrm{C} 2$ & $\mathrm{~J} 014430.48+010052.3$ & 18.4 & & & & & & & & & & \\
\hline $\mathrm{S} 3 \mathrm{C} 2$ & SDSS J0 & 18.334 & & & 16.5 & & & & -17 & 73 & & 90.9 \\
\hline $\mathrm{S} 3 \mathrm{C} 2$ & $.14+291410.5$ & 19.415 & 18.037 & 17.357 & 17.137 & 17.002 & 1.260 & 0.067 & -155.1 & -2.8 & -72.8 & 86.9 \\
\hline $\mathrm{S} 3 \mathrm{C} 2$ & SDSS J075917.15+191709.9 & 18.188 & 17.235 & 16.879 & 16.723 & 16.694 & 1.972 & 0.106 & 241.7 & -114.7 & -65.6 & 84.7 \\
\hline $\mathrm{S} 3 \mathrm{C} 2$ & SDSS J084011.10+130755.4 & 18.854 & 17.7 & 17.174 & 16.948 & 16.869 & 1.483 & 0.087 & 307.7 & -184.6 & -52.9 & 73.8 \\
\hline $\mathrm{S} 3 \mathrm{C} 2$ & SDSS J085412.39+054042.3 & 20.205 & 18.708 & 18.048 & 17.784 & 17.674 & 1.771 & 0.111 & 305.7 & -138.6 & -59.7 & 91.8 \\
\hline $\mathrm{S} 3 \mathrm{C} 2$ & SDSS J111556.02+103009.7 & 15.635 & 14.821 & 14.475 & 14.344 & 14.35 & 0.691 & 0.095 & 185.2 & 90.8 & -69.9 & 91.5 \\
\hline $\mathrm{S} 3 \mathrm{C} 2$ & SDSS J204541.61-050231.6 & 18.002 & 17.164 & 16.814 & 16.666 & 16.569 & 1.863 & 0.102 & -282.1 & -91.7 & -66.5 & 93.5 \\
\hline $\mathrm{S} 3 \mathrm{C} 2$ & SDSS J214325.56-001037.6 & 17.174 & 16.345 & 16.014 & 15.927 & 15.875 & 1.465 & 0.108 & -310.66667 & -174.2 & -52.8 & 82.8 \\
\hline $\mathrm{S} 3 \mathrm{C} 3$ & SDSS J003043.47-002056.2 & 18.002 & 17.161 & 16.8 & 16.679 & 16.669 & 1.992 & 0.144 & -161.2 & -148.6 & -20.0 & 103.3 \\
\hline $\mathrm{S} 3 \mathrm{C} 3$ & SDSS J003837.46-001822.8 & & & 16.605 & & & & & -222.5 & 173.8 & & 96.6 \\
\hline $\mathrm{S} 3 \mathrm{C} 3$ & SDSS J080541.88+065800.1 & & & & & & & & 303 & -160.2 & & 103.7 \\
\hline $\mathrm{S} 3 \mathrm{C} 3$ & SDSS J083752.33+422801.9 & & 17.205 & & & & & & 162.9 & -120.8 & & 106.8 \\
\hline $\mathrm{S} 3 \mathrm{C} 3$ & SDSS J101409.40+395431.0 & 17.446 & 16.601 & 16.246 & 16.118 & 16.123 & 1.554 & 0.126 & 59.9 & 64.8 & -44.5 & 111.0 \\
\hline $\mathrm{S} 3 \mathrm{C} 3$ & SDSS J162108.52+010433.3 & 17.746 & 16.693 & 16.282 & 16.112 & 16.045 & 1.378 & 0.137 & 80.8 & 112.0 & -36.2 & 112.1 \\
\hline $\mathrm{S} 3 \mathrm{C} 3$ & SDSS J213146.48+120942.4 & 17.96 & 16.916 & 16.465 & 16.299 & 16.225 & 1.354 & 0.083 & -306.5 & -102.7 & -46.6 & 106.1 \\
\hline
\end{tabular}

Notes. The last two entries in the bottom of the list are stars that appear in both " $\mathrm{S}_{3}$ " and "C2," and both " $\mathrm{S}_{3}$ " and " $\mathrm{C} 3$," respectively. The reason is that our selection criteria for "C2," "C3," and " $\mathrm{S}_{3}$ " show some overlap. Also, we already noted in Sections 7.4 and 7.6 that a connection between these streams probably exists. 


\section{REFERENCES}

Abadi, M. G., Navarro, J. F., Steinmetz, M., \& Eke, V. R. 2003, ApJ, 597, 21 Abazajian, K., et al. 2009, ApJS, in press (arXiv:0812.0649)

Allende Prieto, C., et al. 2008, AJ, 136, 2070

An, D., et al. 2008, ApJS, 179, 326

Antoja, T., Figueras, F., Fernández, D., \& Torra, J. 2008, A\&A, 490, 135

Arifyanto, M. I., \& Fuchs, B. 2006, A\&A, 499, 533

Binney, J., \& Tremaine, S. 1987, Galactic Dynamics (Princeton: Princeton Univ. Press)

Bochanski, J. J. 2008, PhD Thesis, Univ. Washington

Brook, C. B., Kawata, D., \& Gibson, B. K. 2004, in ASP Conf. Ser. 327, Satellites and Tidal Streams, ed. F. Prada, D. Martinez-Delgado, \& T. Mahoney (San Francisco, CA: ASP), 100

Carollo, D., et al. 2007, Nature, 450, 1020

Chereul, E., \& Grenon, M. 2001, in ASP Conf. Ser. 228, Dynamics of Star Clusters and the Milky Way, ed. S. Deiters et al. (San Francisco, CA: ASP), 398

Chiba, M., \& Beers, T. C. 2000, AJ, 119, 2843

Choi, J.-W., Weinberg, M. D., \& Katz, N. 2007, MNRAS, 381, 987

Clem, J.-L., Vanden Berg, D. A., \& Stetson, P. B. 2008, AJ, 135, 682

De Silva, G. M., et al. 2007, AJ, 133, 694

De Simone, R., Wu, X., \& Tremaine, S. 2004, MNRAS, 350, 627

Dehnen, W. 1998, AJ, 115, 2384

Dehnen, W. 2000, AJ, 119, 800

Dekker, E. 1976, Phys. Reports, 24, 315

Dettbarn, C., Fuchs, B., Flynn, C., \& Williams, M. 2007, A\&A, 474, 857

Dinescu, D. I. 2002, in ASP Conf. Ser. 265, $\omega$ Centauri, A Unique Window into Astrophysics, ed. F. van Leeuwen, J. D. Hughes, \& G. Piotto (San Francisco, CA: ASP), 365

Duquennoy, A., \& Mayor, M. 1991, A\&A, 248, 485

Eggen, O. J. 1996, AJ, 112, 1595

Famaey, B., Jorissen, A., Luri, X., Mayor, M., Udry, S., Dejonghe, H., \& Turon, C. 2004, A\&A, 430, 165

Finlator, K., et al. 2000, AJ, 120, 2615

Fukugita, M., et al. 1996, AJ, 111, 1748

Fux, R. 2001, A\&A, 373, 511

Girardi, L., Bressan, A., Bertelli, G., \& Chiosi, C. 2000, A\&AS, 141, 371

Girardi, L., Grebel, E. K., Odenkirchen, M., \& Chiosi, C. 2004, A\&A, 422, 205

Gould, A. 2003, ApJ, 592, L63

Grillmair, C. J., \& Dionatos, O. 2006, ApJ, 641, L37

Gunn, J. E., et al. 1998, AJ, 116, 3040

Gunn, J. E., et al. 2006, AJ, 131, 2332
Harris, W. E. 1996, AJ, 112, 1487

Helmi, A., \& de Zeeuw, P. T. 2000, MNRAS, 319, 657

Helmi, A., Navarro, J. F., Nordström, B., Holmberg, J., Abadi, M. G., \& Steinmetz, M. 2006, MNRAS, 365, 1309

Helmi, A., \& White, S. D. M. 1999, MNRAS, 307, 495

Helmi, A., White, S. D. M., de Zeeuw, P. T., \& Zhao, H. 1999, Nature, 402, 53

Hogg, D. W., Finkbeiner, D. P., Schlegel, D. J., \& Gunn, J. E. 2001, AJ, 122, 2129

Holmberg, J., Flynn, C., \& Portinari, L. 2006, MNRAS, 367, 449

Ivezić, Ž., et al. 2001, AJ, 122, 2749

Ivezić, Ž., et al. 2003, Mem. Soc. Astron. Ital., 74, 978

Ivezić, Ž., et al. 2008, ApJ, 684, 287

Juric, M., et al. 2008, ApJ, 673, 864

Kalnajs, A. J. 1991, in Dynamics of Disc Galaxies, ed. B. Sundelius (Göteborg: Göteborg Univ.), 323

Kepley, A. A., et al. 2007, AJ, 134, 1579

Klement, R. 2009, Stellar Phase-Space Structure and Dynamics in the Solar Neighbourhood: Kinematic Studies of Nearby Stars (Suetwestdeutscher: Verlag)

Klement, R., Fuchs, B., \& Rix, H. W. 2008, ApJ, 685, 261

Lada, C. J. 2006, ApJ, 640, L63

Lee, Y. S., et al. 2008a, AJ, 136, 2022

Lee, Y. S., et al. 2008b, AJ, 136, 2050

Lindblad, B. 1925, ApJ, 62, 191

Lupton, R. H., et al. 2002, Proc. SPIE, 4836, 350

Mayor, M. 1972, A\&A, 18, 97

Munn, J. A., Monet, D. G., \& Levine, S. E. 2008, AJ, 136, 895

Navarro, J. F., Helmi, A., \& Freeman, K. C. 2004, ApJ, 601, L43

Odenkirchen, M., et al. 2001, ApJ, 548, L165

Peñarrubia, J., Benson, A. J., Martínez-Delgado, D., \& Rix, H. W. 2006, ApJ, 645,240

Pier, J. R., et al. 2003, AJ, 125, 1559

Proctor, R. A. 1869, R. Soc. London Proc. Ser. I, 18, 169

Quillen, A. C., \& Minchev, I. 2005, AJ, 130, 576

Quinn, P. J., \& Goodman, J. 1986, ApJ, 309, 472

Quinn, P. J., et al. 1993, ApJ, 403, 74

Re Fiorentin, P., Helmi, A., Lattanzi, M. G., \& Spagna, A. 2005, A\&A, 439, 551

Sellwood, J. A., \& Binney, J. J. 2002, MNRAS, 336, 785

Soubiran, C., Bienaymé, O., \& Siebert, A. 2003, A\&A, 398, 141

Tucker, D. L., et al. 2006, Astron. Nachr., 327, 821

Vandenberg, D. A., \& Clem, J. L. 2003, AJ, 126, 778

Yanny, B., et al. 2009, AJ, 137, 4377

York, D. G., et al. 2000, AJ, 120, 1579 